\title{
One-pot synthesis of triazines as potential agents affecting cell differentiation
}

\author{
Thomas Linder $^{1} \cdot$ Michael Schnürch $^{1}$ (D) $\cdot$ Marko D. Mihovilovic $^{1}$
}

Received: 8 April 2018/Accepted: 23 April 2018/Published online: 15 May 2018

(C) The Author(s) 2018

\begin{abstract}
This paper outlines the synthesis of a number of structural analogs of 3-[(4,6-diphenoxy-1,3,5-triazin-2-yl)amino]benzoic acid which represent compounds with potential cardiogenetic activity. A one-pot protocol was developed for swift functionalization of the 1,3,5-triazine core without the need of isolating intermediates. The developed route starts from readily available 2,4,6-trichloro-1,3,5-triazine, displacing the chlorine atoms sequentially by aryloxy, arylamino, or arylthio moieties to enable access to molecules with three different substituents of this type in good yields. To facilitate purification, tert-butyl, methyl, and ethyl ester derivatives of the target compounds were initially synthesized. The tertbutyl esters could be readily hydrolyzed to the desired compounds, while reduction of the methyl and ethyl esters gave the corresponding benzylic alcohols in high yields, thereby expanding the substrate scope for future relevant cell assays.
\end{abstract}

\section{Graphical abstract}

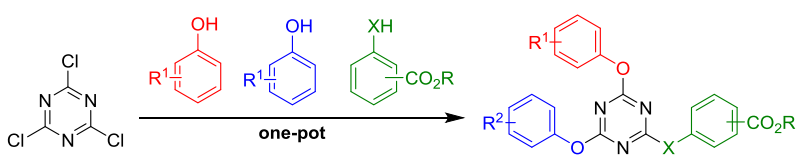

Keywords Cell differentiation · Triazine $\cdot$ One-pot synthesis $\cdot$ Nucleophilic substitution

\section{Introduction}

During the development of animals and humans from a zygote to a multicellular biological system, the organism needs to grow by cell fission and dedicate newly formed cells to particular purposes. The process that converts those cells of the early stages of life (the embryonic stem cells) to specialized tissue is known as cell differentiation. Apart from a merely biological interest, the subject is of great importance to the medical sciences, as it promises to hold the key for replacing damaged or lost tissue and organs [1-5].

Michael Schnürch

michael.schnuerch@tuwien.ac.at

1 Institute of Applied Synthetic Chemistry, TU Wien, Getreidemarkt 9/163, 1060 Vienna, Austria
Cardiovascular diseases are among the most important causes of death globally [6] and an estimated average number of 15 years of life are lost because of a myocardial infarction [7]. As about $34 \%$ of the patients experiencing a coronary attack will die due to this event, these figures point out the importance of new methods for cardiac repair. Apart from replacement by tissue transplant, the restoration by activation of resident (cardiac) stem cells or regeneration by the formation of cardiomyocytes from progenitor or stem cells has been given much attention [8-10]. This is particularly important in the case of renewing dead cardiomyoblasts in the wage of a condition such as myocardial infarction, because the mammalian (i.e., human) heart responds to tissue damage by scarring rather than regeneration [11]. Human embryonic stem cells have shown the potential to develop into cardiomyocytes in vitro. One approach is gene therapy, which involves the use of viral vectors for genetic manipulation and makes it 
difficult to achieve approval by health regulating bodies for clinical use [12, 13]. Similarly, the use of viruses to obtain induced pluripotent stem (iPS) cells from differentiated cells for reprogramming also raises safety issues, while the efficiency of plasmids for this purpose is extremely low [14]. The use of small organic molecules to trigger cell differentiation represents an alternative, which could be carried out ex vivo and the so-formed cardiomyocytes subsequently implanted.

In 2004, a screen with mouse embryonic carcinoma P19 cells showed that certain 2-pyrimidinamines, termed cardiogenols A-D (Fig. 1) up-regulated the expression of an artificial gene containing the promoter for the atrial natriuretic factor (ANF) [15]. This is a polypeptide hormone, synthesized and excreted primarily by cardiomyocytes, and therefore considered a cardiac-specific marker protein $[16,17]$. Considerable research has been conducted in our group to substantiate the cardiogenetic potential of these molecules, as well as finding new ones with promising results in the relevant biological screens (as exemplified in Fig. 2 top) [18-20].

Retinoic acid, which is an active metabolite of retinol (vitamin A) and occurs naturally in the all-trans (atRA) and 9-cis form (Fig. 2 bottom), has been shown to take part in a multitude of physiological functions, notably during the development of the organism and the proliferation of cells [21].

Among these are the inhibition of the serotonin-activated proliferation of aortic smooth muscle cells in primary culture (as shown in dogs) and inhibition of cell proliferation in cultivated chick embryonic vascular smooth muscle cells $[22,23]$, to the extent that retinoids, such as atRA, have been used clinically for anti-cancer treatments and therapies in dermatology [24]. On the other hand, there have been indications that retinoic acid may enhance, rather than suppress, proliferation of smooth muscle cells [25]. Examples like these illustrate that the physiological effects of retinoic acid are very complex and highly dependent on the concentration at which it is applied, as well as the developmental stage of the cell at the time of exposure.

More important to this work, however, is the finding that retinoids generally inhibit cell proliferation and promote cardiac differentiation of stem cells [26]. While applying small molecules for heart muscle formation is a promising approach, applying retinoic acid itself to stem cells has multiple issues. Given its polyolefinic structure, it is rather reactive in the presence of oxygen and light [27]. It is also isomerized under physiological conditions and enters the metabolism of the cell [28]. As indicated above, this natural compound also fulfills many other roles in cell biochemistry and responses of the cell exposed to it depend crucially on the concentration and the state of cell, because its interaction is unlikely to be limited to one target only [29, 30]. This aggravates screening and makes the availability of synthetic retinoids highly desirable.

Recently, it was determined that the heterocyclic compound 3-[(4,6-diphenoxy-1,3,5-triazin-2-yl)amino]benzoic acid (DTAB, compound 4a, Fig. 3) is an RAR $\beta$ and RAR $\gamma$ selective ligand, activating retinoic acid signaling [31]. This compound served as a starting point for the present work, in which a method was developed which enables the preparation of compounds with this scaffold from 2,4,6trichloro-1,3,5-triazine in a sequential, one-pot fashion (Fig. 3), making use of the step-wise reactivity of the chloride substitution process.

Although not strictly analogous to the pyrimidine compounds shown in Figs. 1 and 2, this addition of a nitrogen atom to the core ring structure (by shifting from the pyrimidine to the 1,3,5-triazine heterocycle) extends the scaffold range in a logical bioisosteric modification and complements the previous studies already conducted in the area of cardiogenesis in our group.<smiles>[Y]Nc1nccc(NCCO)n1</smiles>

Cardiogenol $(\mathrm{Cg})$<smiles>O=S(=O)(N/N=C/c1cc(Br)ccc1O)c1ccccc1</smiles><smiles>CC(C)(C)c1ccc(Nc2ccccc2)cc1</smiles><smiles>COc1ccc(C)cc1</smiles><smiles>[B]C(C)(C)c1ccc(Oc2ccccc2)cc1</smiles><smiles>[2H]C(Cl)(Cl)c1ccc(/C=C/c2ccccc2)cc1</smiles>

Fig. 1 Cardiogenols and Shz-1 as literature-known compounds with cardiomyogenic activity 
<smiles>COc1ccc(Nc2cc(Nc3ccc(OC)cc3)ncn2)cc1</smiles>

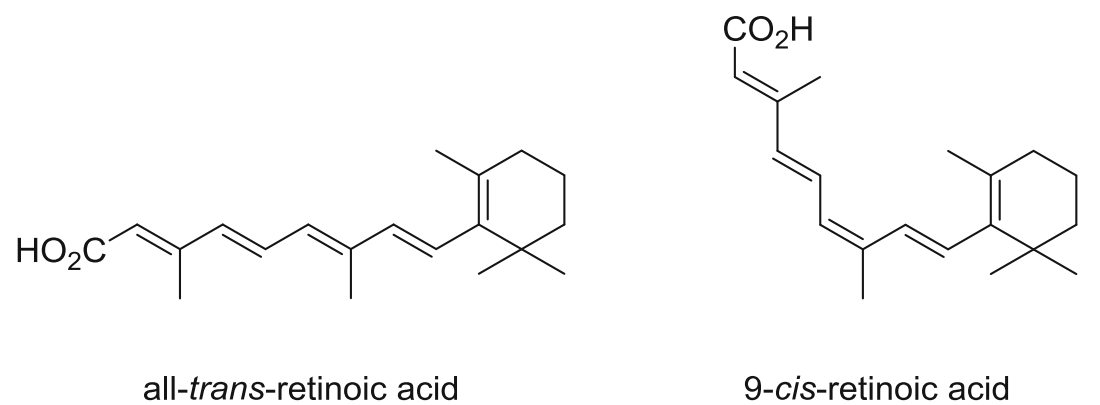

Fig. 2 Examples of compounds with cardiomyogenic activity. Top: synthesized in our group. MK 142 could be shown to produce beating heart muscle cells from W4 embryonic stem cells. Bottom: Retinoic acid isomers

Fig. 3 Parent literature compound and the envisioned synthetic route<smiles>O=C(O)c1cccc(Nc2nc(Oc3ccccc3)nc(Oc3ccccc3)n2)c1</smiles><smiles>Clc1nc(Cl)nc(Cl)n1</smiles>

1<smiles>[R]O[R](=O)c1cccc([X])c1</smiles>

$\mathrm{R}=\mathrm{H}, \mathrm{Me}$, tert-Bu $\mathrm{X}=\mathrm{NH}, \mathrm{S}$

\section{Results and discussion}

Symmetrically trisubstituted triazines can be prepared by protic or Lewis acid- or base-catalyzed cyclotrimerization of nitriles or acid-catalyzed cyclocondensation of imidic esters (Scheme 1) [32].

1,3,5-Triazines with three different substituents are accessible by condensation of acylamidines (themselves prepared from amides and amide acetals) with other amidines (Scheme 1) [28].
2,4,6-Trichloro-1,3,5-triazine (cyanuric chloride, $\mathbf{1}$ ) is an electron-poor heterocycle and behaves as a heterocyclic acid chloride analog, with its reactive chlorine atoms being easily substituted in $\mathrm{S}_{\mathrm{N}} \mathrm{Ar}$ reactions. Therefore, it can serve as starting material for (differently) substituted 1,3,5trazines, where the substituents are linked via nucleophilic heteroatoms, such as oxygen, nitrogen, and sulfur.

To gain experience with nucleophilic aromatic substitution on the 1,3,5-triazine scaffold, a known procedure to produce 2-phenoxy-4,6-dichloro-1,3,5-triazine (2a) was repeated [33]. In this procedure, 2,4,6-trichloro-1,3,5- 
Scheme 1

3<smiles>[R]C#N</smiles>

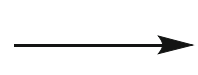

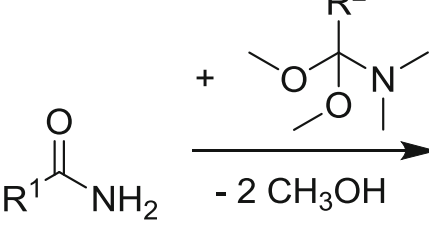<smiles>[R]C(N)=O</smiles><smiles>[R]c1nc([R])nc([R])n1</smiles><smiles>CC=C(C)[R](C)=O</smiles><smiles>[R]C(=O)N=C([R])NC</smiles><smiles>[R]C(=N)N</smiles>

$-\mathrm{H}_{2} \mathrm{O}$
$3 \mathrm{R}$<smiles>[R]OC([R])=N</smiles>

triazine was combined with one equivalent of phenol and $\mathrm{N}, \mathrm{N}$-diisopropylethylamine (DIPEA) in THF at $0{ }^{\circ} \mathrm{C}$ (Scheme 2 top). While the authors state that no purification was required (short of removing the amine hydrochloride by filtration) to afford $\mathbf{2 a}$ in $92 \%$ yield, GC-MS analysis revealed that about $10 \%$ of the disubstituted triazine $\mathbf{3 a}$ was formed, as well. Although this could be removed by recrystallization, such a byproduct ratio would have a negative impact on the one-pot procedure in which three sequential nucleophilic replacements were aimed at without any work-up of intermediates. Thus, on repetition of this synthesis, the reaction temperature was lowered to $15{ }^{\circ} \mathrm{C}$ initially and the reaction time was extended to allow for complete conversion, which avoided formation of byproduct and gave 2a in 95\% without further purification (Scheme 2 top).

This reaction proceeds swiftly in the beginning, with the appearance of the hydrochloride salt after a few minutes. Since combining the two components under basic conditions is noticeably exothermic, it was decided to add the phenolic compounds as THF solutions to ensure the reproducibility of byproduct suppression. Disubstituted 3a was prepared in this way, adding two equivalents in succession. After the amount of the mono-substituted intermediate 2a had decreased to less than 2\% (GC-MS), the reaction mixture was worked up and gave 3a in $78 \%$ after purification (Scheme 2 bottom).

In general, neither of these two intermediates is very stable; they decompose visibly if stored at room

Scheme 2

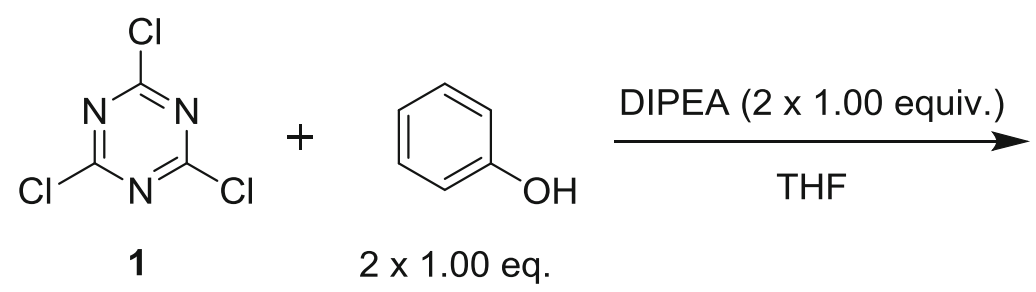<smiles>Clc1nc(Cl)nc(Oc2ccccc2)n1</smiles>

$2 a, 95 \%$<smiles>Clc1nc(Oc2ccccc2)nc(Oc2ccccc2)n1</smiles>

$3 a, 78 \%$ 
temperature for a period of a few months. Unlike the actual target trisubstituted triazines, the disubstituted compounds are also attacked by DMSO, which precludes direct NMR shift comparison in the same solvent, because the latter are generally not soluble enough in, e.g., $\mathrm{CDCl}_{3}$ (trichlorotriazine itself reacts vigorously with DMSO, by attack of the oxygen) [34]. This actually speaks in favor of a method which omits isolation and purification of such reactive intermediates and makes a one-pot protocol all the more appealing.

An unexpected behavior occurred when two different aryloxy residues were attempted to be incorporated into the triazine scaffold. In the first step, 4-chlorophenol (an electron-deficient phenol) was reacted with starting material 1, and the reaction was checked for completion by TLC and GC-MS. Then, with addition of phenol in the second step, three products emerged (Scheme 3): in addition to expected 3b, compounds $\mathbf{3 c}$ and $\mathbf{3 a}$ were also detected, in the approximate ratio of 8:2:1. A very similar product distribution was obtained when 2- or 3-chlorophenol was used in step one. By contrast, starting from purified $\mathbf{2 a}$ and reacting it with 4-chlorophenol under the same conditions, almost none of the undesired products were formed (ratio of $97: 2: 1)$

These findings can be readily explained by looking at the electronic features of the applied phenols. Owing to the electron-withdrawing substituent, chlorophenol(s) can also serve as a leaving group and remain present in the reaction mixture after replacement by phenol. In this displacement reaction, $\mathbf{2 a}$ is formed as an intermediate. Both $\mathbf{2 b}$ and $\mathbf{2 a}$ can then form substitution products with either phenol (added to the mixture for the second step) or chlorophenol (liberated in the displacement) and, hence, lead to the observed product distribution. This has two implications: if both electron-rich(er) and electron-poor(er) phenoxy substituents have to be incorporated at the triazine core, then the order of addition of the two phenols is important: the electron-richer(er) has to be added prior to the electronpoor(er). Knowing this, the final step of the sequence could be investigated.

A preliminary experiment showed that 3-aminobenzoic acid reacts with $\mathbf{3 a}$ under much the same conditions (DIPEA as the base) as in the preparation of $\mathbf{2 a}$ and $\mathbf{3 a}$ to give the parent literature compound $4 \mathbf{a}$ (DTAB, Scheme 4).

As a somewhat troubling property, it was found that none of these trisubstituted triazine compounds (with or without the presence of a carboxylic acid function) could be detected in either GC or GC-MS analysis, limiting reaction control to TLC analysis. In general, the triazinylaminobenzoic acids were crystalline materials, sparinglyto-moderately soluble in common organic solvents (e.g., very low solubility in $\mathrm{CHCl}_{3}, \mathrm{MeOH}, \mathrm{Et}_{2} \mathrm{O}, \mathrm{MeCN}$; best solubility was found with 1,4-dioxane). It, therefore, appeared promising to prepare a variety of these compounds in one pot by starting from trichlorotriazine, incorporating first two different phenoxy substituents and subsequently adding the amine compound.

While the reaction itself worked as expected, a severe purification problem occurred: as chromatographic separation of carboxylic acids was not feasible, re-crystallization remained the only means of purification. Because the step-wise reactivity of trichlorotriazine in the nucleophilic substitution was neither fully selective, nor complete, unwanted other triazinyl benzoic acids, mostly those with two identical aryloxy substituents, were also obtained. These persisted in the $5-10 \%$ range as judged by HPLC analysis after re-crystallization from various solvents

Scheme 3

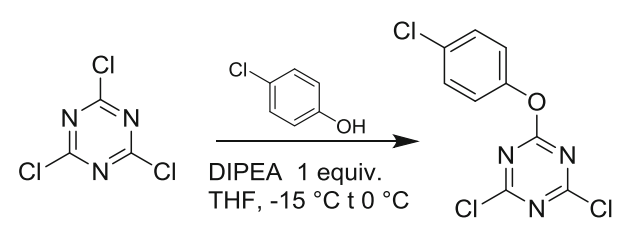

2

2b
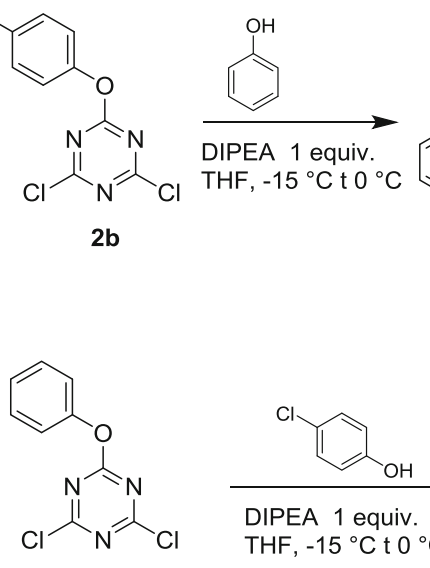

$2 a$

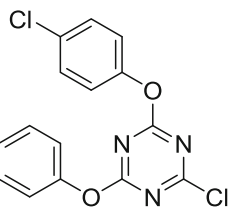

$3 b$

8
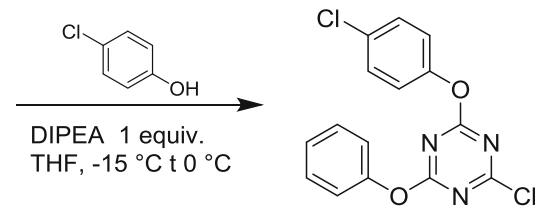

$3 b$

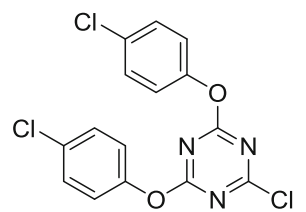

3c

2

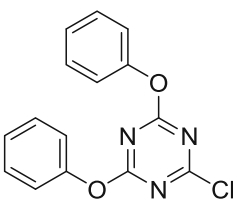

$3 a$

1 
Scheme 4

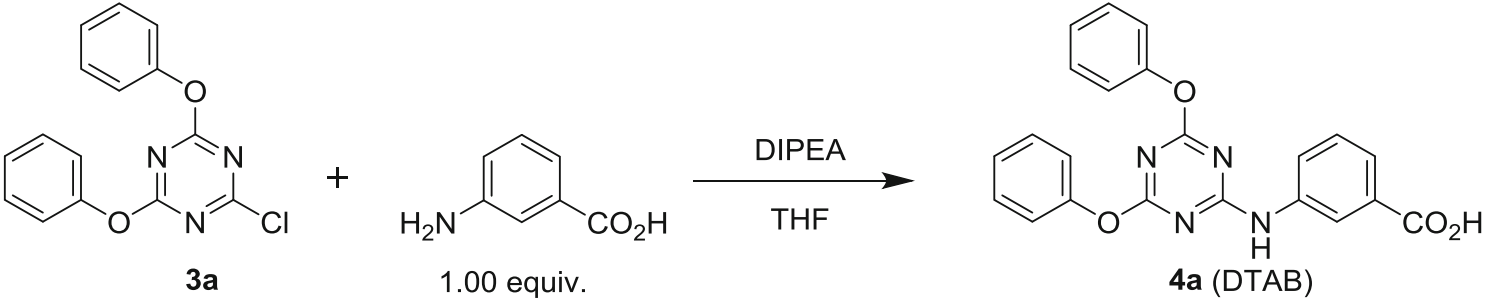

$\left(\mathrm{CHCl}_{3} / n\right.$-hexane, toluene, and EtOH). Therefore, target compounds could not be purified to a satisfactory degree.

In light of these purification issues, carboxylic acids were masked as their methyl (and, in one case, ethyl) esters, i.e., retaining the one-pot protocol but using an alkyl aminobenzoate in the third substitution step (Scheme 5). This opened the possibility for chromatographic separation.

To reduce disubstitution in the first nucleophilic displacement even further, the solutions containing the phenols and the base were also cooled to the reaction temperature $\left(-35^{\circ} \mathrm{C}\right.$ ) prior to dropwise addition (at the reported temperature of $0{ }^{\circ} \mathrm{C}[20,35]$, the reaction is less selective, as stated above). In general, care needs to be taken as to drive the reaction as far to completion as possible, however, without losing selectivity. This is why the reaction times tended to be very long (up to $47 \mathrm{~h}$ ), while the reaction temperature was not taken beyond $+40{ }^{\circ} \mathrm{C}$. The results are summarized in Scheme 5.

The reactions, which were carried out using readily available phenols proceeded very well in most cases. Various electron-rich and -poor phenols were used to investigate the scope of this method, also beyond common pharmacophores such as chloro and methoxy substituents. As for the performance of the reaction, there is no significant difference in yields of compounds bearing electron donating $p$-tert-butyl and $p$-methoxy, versus electronwithdrawing $p$-, $m$-, $o$-chloro, $p$-cyano, and $m$-nitro substituents. One exception is compound 5l: the second nucleophilic substitution with 5-bromo-2-hydroxybenzaldehyde was not complete, even after $46 \mathrm{~h}$, and considerable amount of the starting material was recovered by column chromatography. In addition, purification was plagued with separating this compound from byproducts as it showed great tailing on the column. Therefore, after two chromatographic runs, only $15 \%$ could be obtained. By contrast, more electron-rich aldehydes, such as vanillin (leading to $\mathbf{5 j}$ and 5k), worked quite well and difficulties with the use of the 3-aminobenzoate in the third step did not arise, probably owing to the low temperature conditions.
Compounds $\mathbf{5 e}$, 5f, and $\mathbf{5 h}$ were first purified by recrystallization. As this did not eliminate byproducts completely (i.e., differently substituted triazines; impurities in the $1-5 \%$ range), subsequent column chromatography was applied for final purification, but the overall decrease in yield was significantly larger than the amount of impurity initially present (Scheme 5, yields before column are in parentheses).

To evaluate the importance of a carboxylic functional group on the aniline moiety in positions 3 and 4 for biological activity, two more compounds were prepared devoid of this functionality. Since the two phenoxy substituents were identical, two equivalents of phenol were used in one go rather than adding them sequentially as in the cases above (Scheme 6). Being a good nucleophile, thiophenol was also incorporated well into the triazine ring.

While 6 is a known compound [36, 37], a literature reference to 7 could not be found. It offers the possibility of another linking group besides $\mathrm{NH}$ and $\mathrm{S}$, by oxidation of the sulfide to the sulfoxide (SO) or the sulfone $\left(\mathrm{SO}_{2}\right)$. This could be used if one wishes to reverse the role of this part of the molecule in hydrogen bonding, switching it from a hydrogen donor to an acceptor.

Next, the obtained benzoic ester products had to be hydrolyzed to the corresponding carboxylic acids. Since methyl and ethyl esters are usually best cleaved by irreversible base hydrolysis, this was attempted first. However, in all the attempts and seemingly irrespective of temperature $\left(-20\right.$ to $\left.+50{ }^{\circ} \mathrm{C}\right)$, the hydroxide ion attacked the electron-deficient triazine carbon bonded to the phenoxy substituents and released the phenol, preferentially the more electron-deficient one. This process also consumed the amount of base available, leaving unhydrolyzed ester behind. In addition, a variation of hydroxide-promoted hydrolysis in a non-aqueous environment did not bring the desired results [38].

On the other hand, the model compounds proved inert towards acid hydrolysis, short of exceptionally harsh conditions leading to its destruction. Since all attempts to cleave the methyl esters proved to be futile, the use of alternative esters was considered. 
One-pot synthesis of triazine as potential agents affecting cell differentiation

1263

Scheme 5<smiles>Clc1nc(Cl)nc(Cl)n1</smiles>
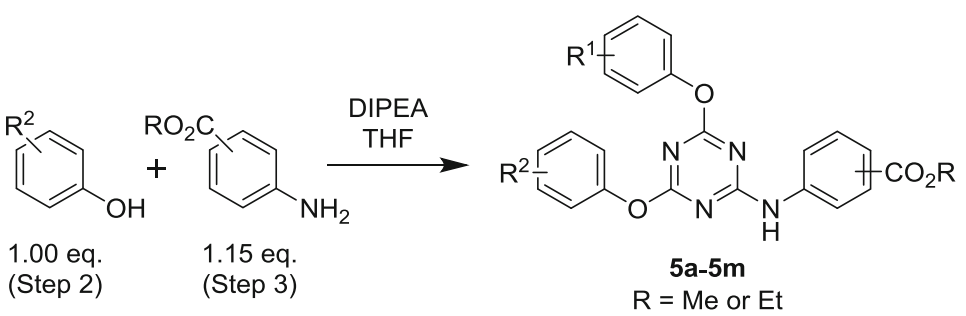

Typical conditions:

step 1: 2,4,6-trichlorotriazine (1.00 equiv.), $\mathrm{R}^{1}-\mathrm{Ph}-\mathrm{OH}$ (1.00 equiv.), DIPEA ( 1.10 equiv.); $-35^{\circ} \mathrm{C}$ for $6 \mathrm{~h}$ step 2: $\mathrm{R}^{2}-\mathrm{Ph}-\mathrm{OH}$ (1.00 equiv.), DIPEA ( 1.60 equiv.); $+35^{\circ} \mathrm{C}$ for $46 \mathrm{~h}$

step 3: aminobenzoate (1.15 equiv.), DIPEA (1.50 equiv.); $+35^{\circ} \mathrm{C}$ for $26 \mathrm{~h}$<smiles>CCOC(=O)c1ccc(Nc2nc(Oc3ccccc3)nc(Oc3ccccc3)n2)cc1</smiles><smiles>CC(=O)c1cccc(Nc2nc(Oc3ccccc3)nc(Oc3ccccc3)n2)c1</smiles>

sa, $95 \%$

Sb, $84 \%$<smiles>COC(=O)c1cccc(Nc2nc(Oc3ccccc3)nc(Oc3ccc(Cl)cc3)n2)c1</smiles><smiles>COC(=O)c1cccc(Nc2nc(Oc3ccccc3)nc(Oc3cccc(Cl)c3)n2)c1</smiles>

Sd, $89 \%$<smiles>COC(=O)c1cccc(Nc2nc(Oc3ccccc3)nc(Oc3ccccc3Cl)n2)c1</smiles>

se, $67 \%(80 \%)$

sc, $81 \%$<smiles>CC(=O)c1cccc(Nc2nc(Oc3ccccc3)nc(Oc3ccc(C(C)(C)C)cc3)n2)c1</smiles>

Sf, $66 \%(80 \%)$<smiles>COc1ccc(Oc2nc(Nc3cccc(C(C)=O)c3)nc(Oc3ccccc3)n2)cc1</smiles>

$5 g, 85 \%$<smiles>COC(=O)c1cccc(Nc2nc(Oc3ccccc3)nc(Oc3ccc(C#N)cc3)n2)c1</smiles>

5 h, $71 \%(86 \%)$<smiles>CC(=O)c1cccc(Nc2nc(Oc3ccccc3)nc(Oc3cccc([N+](=O)[O-])c3)n2)c1</smiles>

$5 i, 69 \%$<smiles>COC(=O)c1cccc(Nc2nc(Oc3ccccc3)nc(Oc3ccc(C=O)cc3OC)n2)c1</smiles><smiles>COC(=O)c1cccc(Nc2nc(Oc3ccc(OC)cc3)nc(Oc3ccc(C=O)cc3OC)n2)c1</smiles><smiles>COC(=O)c1cccc(Nc2nc(Oc3ccc(OC)cc3)nc(Oc3ccc(Br)cc3C=O)n2)c1</smiles><smiles>COC(=O)c1cccc(Nc2nc(Oc3ccc(C#N)cc3)nc(Oc3ccc(OC)cc3)n2)c1</smiles>

$5 \mathrm{~m}, 88 \%$

Springer 
Scheme 6<smiles>Clc1nc(Cl)nc(Cl)n1</smiles>

1<smiles>Oc1ccccc1</smiles>

2.00 eq. (Step 1)<smiles>[X]c1ccccc1</smiles>

1.15 eq. (Step 2)
The tert-butyl ester group is well known to be readily cleaved by acid, because the stable tert-butyl carbenium ion can act as a leaving group on protonation of the adjacent oxygen atom [39]. Moreover, no water is required in this process, the only byproduct is gaseous isobutene (formed by elimination from the carbenium ion) and this deprotection can be carried out under mild conditions. For this reason, 3-tert-butylaminobenzoate was used in a onepot sequence analogously to the synthesis of the methyl esters (Scheme 7).

In an overall view, this reaction produced somewhat lower yields and appeared to be slower (Scheme 7) than the synthesis of the corresponding methyl esters. The tertbutyl group was stable on silica during chromatography. Work-up had to include an aqueous extraction step with diluted hydrochloric acid to remove the 3-tert-butylaminobenzoate, which was used in slight excess $(1.15$ equivalents), because this material had very similar $R_{f}$ values to products $\mathbf{8 a}-\mathbf{8 j}$. This operation was carried out quickly to avoid ester cleavage prior to chromatographic separation-even though the corresponding carboxylic acid was eventually desired, because only the ester could be purified chromatographically. The solution containing the product was then treated with $\mathrm{NaHCO}_{3}$ solution (for neutralization) and brine before evaporation and column chromatography. The compounds also turned out to be generally more soluble and less inclined to crystallize than the corresponding methyl esters because of the non-polar tert-butyl group, although crystalline materials could be obtained in all cases.

As indicated above, the tert-butyl group can be removed easily under moderately strong acidic conditions. The classic removal condition involves the use of trifluoroacetic acid [40] $\left(\mathrm{p} K_{A}=0.23\right)$ [41], but formic acid [42] $\left(\mathrm{p} K_{A-}\right.$ $=3.77$ ) [43] can also be used. This is, therefore, a milder method, which is compatible with a large number of functional groups. First, formic acid, dissolved in $\mathrm{CH}_{2} \mathrm{Cl}_{2}$ was tried at room temperature, but this did not advance the reaction. However, upon heating to $40{ }^{\circ} \mathrm{C}$, the reaction proceeded as observed by TLC after several hours. Finally, it proved a very simple and effective operation to take up

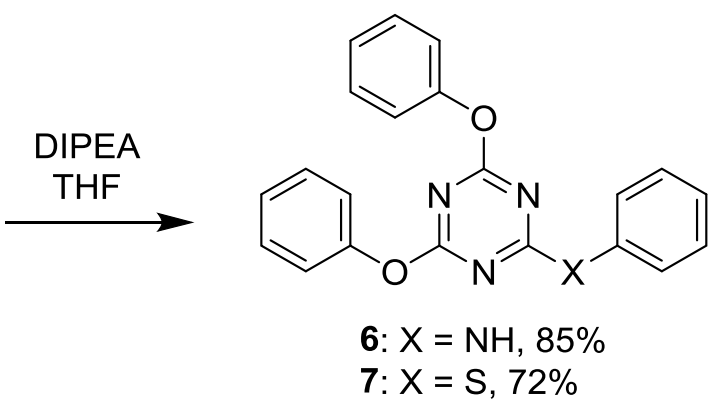

the compound solely in formic acid (with some sonication to aid dissolution) and stir the solution at slightly elevated temperatures $\left(40-50^{\circ} \mathrm{C}\right)$. A large excess of formic acid $\left(4 \mathrm{~cm}^{3}\right.$ for, e.g., $0.1 \mathrm{mmol}$ ) was used to make sure that the intermediately formed and very strongly electrophilic tertbutyl cation would not be likely to react with either the starting material or the product (as is sometimes the case) [44] in, e.g., an electrophilic aromatic substitution reaction of the carbocycles, before it is eliminated to isobutene. In all but one case (compound $\mathbf{4 j}$ ), the cleavage reaction was complete within $1 \mathrm{~h}$.

Purification was not required; work-up was limited to adding diethyl ether after concentrating the reaction product to dryness and to remove the solvent again in vacuo to aid evaporation of the formic acid. Thus, the desired carboxylic acids $\mathbf{4 a}-\mathbf{4 k}$ were obtained in quantitative yields (Scheme 8).

From the viewpoint of biological activity, it is interesting to compare the changes that occur when the carboxylic acid function-common to all structures so far, except $\mathbf{6}$ and 7-is replaced by another group. The hydroxyl functional group of a benzylic alcohol represents such a change; it is also polar and capable of hydrogen bonding. These compounds were obtained by reducing the corresponding methyl and ethyl esters, since the tert-butyl group of compounds in Scheme 7 would be quite resistant to hydride attack. Usually, such a transformation is accomplished by a highly reactive complex metal hydride, notably lithium aluminium hydride. Since it was already obvious that strong nucleophiles are not tolerated very well due to the reactivity of the $\mathrm{C}-\mathrm{O}$ bond of the substituted triazines, a less aggressive method would be highly soughtafter. Based on the higher electronegativity of boron (2.0 of $\mathrm{B}$ vs. 1.6 of $\mathrm{Al}$ ), the frequently used sodium borohydride is a less reactive reducing agent but usually falls short of transforming esters accordingly. However, additional reactivity can be gained by changing the counter-ion to lithium, which offered the prospect of having just the right reductive power to accomplish the task. Literature suggested that the reactivity of the borohydride has been enhanced by the addition of lithium chloride [45], and fast 


\section{Scheme 7}

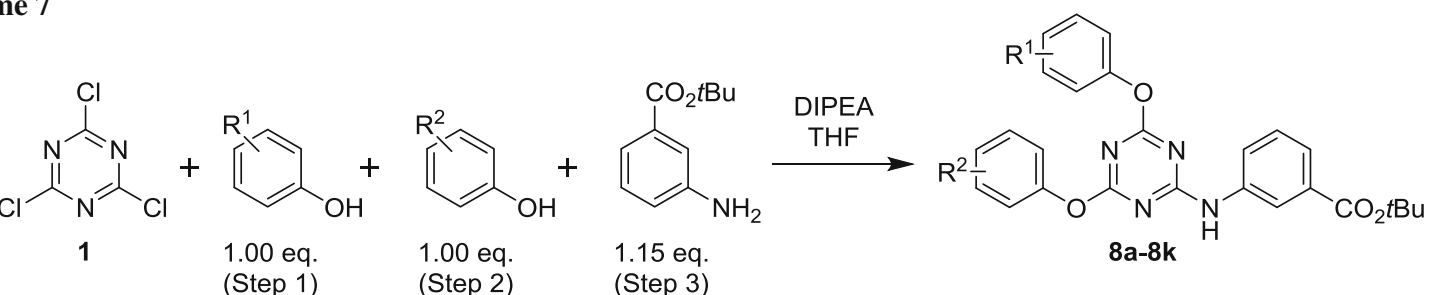<smiles>CCOC(=O)c1cccc(Nc2nc(Oc3ccccc3)nc(Oc3ccccc3)n2)c1</smiles>

8a, $73 \%$<smiles>CCOC(=O)c1cccc(Nc2nc(Oc3ccccc3)nc(Oc3ccccc3Cl)n2)c1</smiles>

$8 d, 77 \%$<smiles>CCOC(=O)c1cccc(Nc2nc(Oc3ccccc3)nc(Oc3ccc(C#N)cc3)n2)c1</smiles>

8g, $33 \%$<smiles>CCOC(=O)c1cccc(Nc2nc(Oc3ccccc3)nc(Oc3ccc(Cl)cc3)n2)c1</smiles>

8b, $89 \%$<smiles>CCOC(=O)c1cccc(Nc2nc(Oc3ccccc3)nc(Oc3ccc(C(C)(C)C)cc3)n2)c1</smiles>

$8 \mathbf{e}, 67 \%$<smiles>CCOC(=O)c1cccc(Nc2nc(Oc3ccccc3)nc(Oc3cccc(Cl)c3)n2)c1</smiles>

8c, $76 \%$<smiles>CCCOC(=O)c1cccc(Nc2nc(Oc3ccccc3)nc(Oc3ccc(OC)cc3)n2)c1</smiles>

8f, $68 \%$<smiles>CCOC(=O)c1cccc(Nc2nc(Oc3ccccc3)nc(Oc3cccc([N+](=O)[O-])c3)n2)c1</smiles>

8 h, $33 \%$<smiles>CCOC(=O)c1cccc(Nc2nc(Oc3ccccc3)nc(Oc3ccc(C=O)cc3OC)n2)c1</smiles>

8i, $55 \%$<smiles>CCCCCOc1ccc(C=O)cc1Oc1nc(Nc2cccc(C(=O)OCC)c2)nc(Oc2ccc(OC)cc2)n1</smiles>

$8 j, 59 \%$<smiles>CCOC(=O)c1cccc(Nc2nc(Oc3ccc(C#N)cc3)nc(Oc3ccc(OC)cc3)n2)c1</smiles>

8k, $57 \%$ reductions with this reagent have been performed by microwave irradiation [46]. Moreover, it has been reported that reducible groups, such as $\mathrm{NO}_{2}$ and $\mathrm{CN}$, can be preserved under lithium borohydride conditions [47].

To test the reduction, compounds $\mathbf{5} \mathbf{c}-\mathbf{5 e}$ were used again (Scheme 9). An initial attempt to carry out such a reduction of $5 \mathbf{e}$ using 1.1 equivalents of $\mathrm{NaBH}_{4}$ in the presence of 2.0 equivalents of $\mathrm{LiCl}$ by the conventional heating at $65^{\circ} \mathrm{C}$ in anhydrous THF did not bring about this transformation after $18 \mathrm{~h}$. Higher temperature $\left(75^{\circ} \mathrm{C}\right.$, and then $90^{\circ} \mathrm{C}$ ) only led to compound decomposition as indicated by multiple spots on TLC. Since also other related conditions did not lead to the desired reduction in acceptable amounts, another reducing agent had to be considered. Based on the idea that increased steric bulk may prevent the hydride ion from attacking the electrophilic triazine carbons, diisobutylaluminium hydride was tried under standard conditions in anhydrous $\mathrm{CH}_{2} \mathrm{Cl}_{2}$ under argon at $-70{ }^{\circ} \mathrm{C}$. 


\section{Scheme 8}

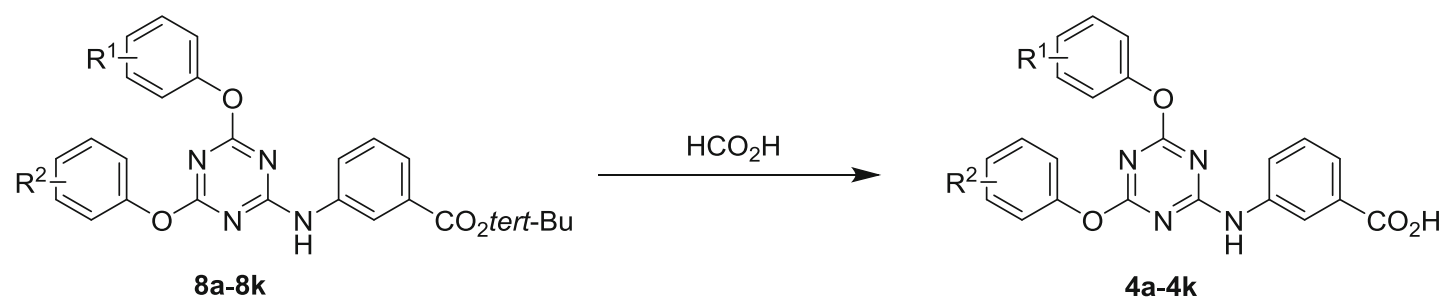<smiles>O=C(O)c1cccc(Nc2nc(Oc3ccccc3)nc(Oc3ccccc3)n2)c1</smiles>

4a, quant.<smiles>Oc1ccccc1</smiles><smiles>O=C(O)c1cccc(Nc2nccnc2Oc2ccccc2Cl)c1</smiles>
4d, quant<smiles>O=C(O)c1cccc(Nc2nc(Oc3ccccc3)nc(Oc3ccc(Cl)cc3)n2)c1</smiles>
4b, quant.<smiles>CC(C)(C)c1ccc(Oc2nc(Nc3cccc(C(=O)O)c3)nc(Oc3ccccc3)n2)cc1</smiles>
4e, quant.<smiles>O=C(O)c1cccc(Nc2nc(Oc3ccccc3)nc(Oc3cccc(Cl)c3)n2)c1</smiles>

4c, quant.<smiles>COc1ccc(Oc2nc(Nc3cccc(C(=O)O)c3)nc(Oc3ccccc3)n2)cc1</smiles>

4f, quant.<smiles>COc1cc(C=O)ccc1Oc1nc(Nc2cccc(C(=O)O)c2)nc(Oc2ccccc2)n1</smiles>

$4 \mathbf{i}$, quant.<smiles>COc1ccc(Oc2nc(Nc3cccc(C(=O)O)c3)nc(Oc3ccc(C=O)cc3OC)n2)cc1</smiles><smiles>COc1ccc(Oc2nc(Nc3cccc(C(=O)O)c3)nc(Oc3ccc(C#N)cc3)n2)cc1</smiles>

4k, quant.

Although a large excess of this reagent (up to 5.1 equivalents) had to be used to complete the reaction relatively quickly (around 1-3 h), the desired conversion could be completed with good yields (Scheme 10). Most importantly, no phenols were detected, and purification was only needed to remove some residual starting material that had escaped TLC analysis (due to its low concentration) during the reaction. However, triazinyl benzoic esters 
Scheme 9<smiles>COC(=O)c1cccc(Nc2nc(Oc3ccccc3)nc(Oc3ccccc3)n2)c1</smiles>

5c: 4-Cl; 5d: 3-Cl; 5e: 2-Cl)
$\mathrm{LiBH}_{4}$ or<smiles>Oc1ccccc1</smiles><smiles>Cc1nc(Nc2cccc(CO)c2)nc(OC2=CC=CI=C=C2)n1</smiles>

$9 c-9 e$

Scheme 10

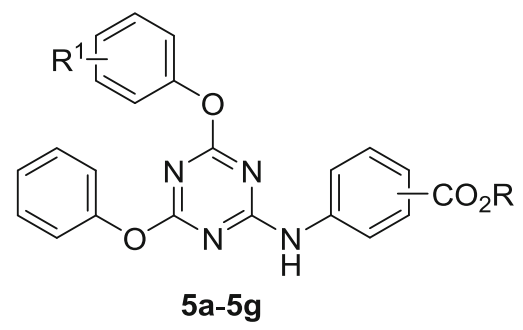

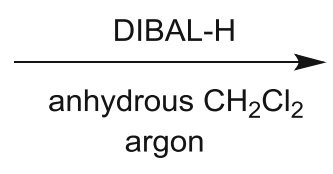<smiles>[R6][Y1]1ccc(Oc2nc(Nc3ccc(CO)cc3)nc(Oc3ccccc3)n2)cc1</smiles><smiles>OCc1ccc(Nc2nc(Oc3ccccc3)nc(Oc3ccccc3)n2)cc1</smiles>

9a, quant.<smiles>OCc1cccc(Nc2nc(Oc3ccccc3)nc(Oc3ccccc3)n2)c1</smiles>

9b, quant.<smiles>OCc1cccc(Nc2nc(Oc3ccccc3)nc(Oc3ccc(Cl)cc3)n2)c1</smiles>

$9 c, 89 \%$<smiles>OCc1cccc(Nc2nc(Oc3ccccc3)nc(Oc3cccc(Cl)c3)n2)c1</smiles>

9d, $78 \%$<smiles>CC(C)(C)c1ccc(Oc2nc(Nc3cccc(CO)c3)nc(Oc3ccccc3)n2)cc1</smiles>

9f, quant.<smiles>OCc1cccc(Nc2nc(Oc3ccccc3)nc(Oc3ccccc3Cl)n2)c1</smiles>

9e, quant.<smiles>COc1ccc(Oc2nc(Nc3cccc(CO)c3)nc(Oc3ccccc3)n2)cc1</smiles>

9g, quant. 
bearing nitro and cyano groups were not attempted, because these functions are both readily reduced, as well.

\section{Conclusion}

In this work, it was demonstrated that derivates of the triazine compound DTAB, $\mathbf{4 a}$, bearing three different substituents, can be prepared in a one-pot protocol. However, if carboxylic acids akin to DTAB are desired, separation problems emerge; and it is, therefore, necessary to mask the carboxylic function to facilitate purification. The compounds in question are still labile toward nucleophiles (hydroxide, hydride, and methoxide), which prevents smooth basic hydrolysis of methyl esters and their reduction to the corresponding benzylic alcohols with lithium or sodium borohydride. Still, they can be converted using the more sterically demanding diisobutylaluminium hydride in good yields. In addition, the triazine scaffold is inert toward acid, which allows the facile and operationally simple acid-promoted cleavage of tert-butyl esters of the corresponding carboxylic acids, affording the latter quantitatively. Biological results have been recently disclosed in a patent application [48, 49].

\section{Experimental}

Chemicals: Unless otherwise noted, chemicals were purchased from commercial suppliers and used without further purification. DIBAL-H solution in $n$-hexane was reactiontitrated according to a literature procedure $(3 \times)$ and the content of active DIBAL-H was determined by standard ${ }^{1} \mathrm{H}$ NMR spectroscopy [50]. Dry solvents were obtained by passing pre-dried material through a cartridge containing activated alumina (solvent dispensing system) and stored under $\mathrm{N}_{2}$. Microwave reactions were performed using a Biotage Initiator 2.5 laboratory microwave device. Chromatography: Flash column chromatography was performed on Merck silica gel $60(40-63 \mu \mathrm{m})$. Separations were carried out either using a Büchi Sepacore system (MPLC) or by hand column (as noted). For thin-layer chromatography (TLC), aluminium backed Merck silica gel 60 with fluorescence indicator $\mathrm{F}_{254}$ was used. For preparative TLC, Analtech Uniplate silica gel GF $(20 \times 20 \mathrm{~cm}, 1000 \mu \mathrm{m})$ glass-backed plates with fluorescence indicator UV254 were used. Distillation: Kugelrohr distillation was carried out using a Büchi GKR-51 apparatus. Melting points were determined using a Kofler-type Leica Galen III micro hot stage microscope or a Stanford Research Systems MPA100 OptiMelt Automatic Melting Point System. Data are given in $0.5{ }^{\circ} \mathrm{C}$ intervals. GC-MS: GC-MS runs were performed on a Thermo Finnigan Focus GC/DSQ II using a standard capillary column BGB $5(30 \mathrm{~m} \times 0.32 \mathrm{~mm}$ ID $)$ and applying the following standardized temperature profile: 2 min at $100{ }^{\circ} \mathrm{C} / \mathrm{ramp} 18{ }^{\circ} \mathrm{C} \mathrm{min}{ }^{-1}$ until $280{ }^{\circ} \mathrm{C} / 5 \mathrm{~min}$ at $280{ }^{\circ} \mathrm{C}$. Electron ionization was used $(70 \mathrm{eV})$; all fragment signals $(\mathrm{m} / \mathrm{z})$ at/over mass 100 and at/over $10 \%$ relative intensity are indicated. NMR spectroscopy: NMR spectra were recorded from DMSO- $d_{6}$ or $\mathrm{CDCl}_{3}$ solutions on a Bruker AC $200(200 \mathrm{MHz})$ or a Bruker Avance UltraShield (400 MHz) spectrometer (as indicated), and chemical shifts are reported in ppm relative to the nominal residual solvent signals [51]: $\mathrm{CDCl}_{3}: \delta=7.26 \mathrm{ppm}\left({ }^{1} \mathrm{H}\right), \delta=77.16 \mathrm{ppm}$ $\left({ }^{13} \mathrm{C}\right)$; DMSO- $d_{6}: \delta=2.50\left({ }^{1} \mathrm{H}\right), \delta=39.52\left({ }^{13} \mathrm{C}\right)$. DEPT135 or $J$-modulation pulse sequences (APT) were used to aid in the multiplet assignment in the ${ }^{13} \mathrm{C}$ spectra.

Numbering of the atoms in the synthesized products parallels the priority of the individual cycles of the molecules, as inferred from systematic nomenclature. Therefore, the hydrogen and carbon positions belonging to the ring with the highest priority are referred to as $\mathrm{H} 1, \mathrm{H} 2, \mathrm{H} 3, \ldots$ and $\mathrm{C} 1, \mathrm{C} 2, \mathrm{C} 3, \ldots$, respectively. The numbers designate the order of the atoms within a particular ring system. The atoms in the second ring are indicated with one prime ( $\mathrm{H} 1^{\prime}$,

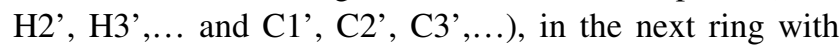
two primes, etc. However, the core 1,3,5-triazine cycle is given highest priority in all cases. This is done regardless that a compound may actually bear its name-giving functional group on one of the lower priority rings, as the suffix ... acid implies for the example, as shown in Fig. 4.

In addition, all compounds are drawn in such a way that they follow the ', ", ," order of the second, third, and fourth ring in the clockwise manner as shown. Identified signals were marked with an asterisk $(*)$ when assignment was equivocal.

\section{NMR characteristics of 2,4,6-trisubstitued 1,3,5- triazines}

While 2,4,6-trisubstituted 1,3,5-triazines present rather ordinary proton NMR spectra, a noteworthy aspect became apparent in ${ }^{13} \mathrm{C}$ spectra: carbons $\mathrm{C} 4$ and $\mathrm{C} 6$, such as in compound 5c, (Fig. 5; i.e., those adjacent to the aryloxy groups) appeared at low field in the $170-173 \mathrm{ppm}$ range relative to TMS. They gave rather broad and also low

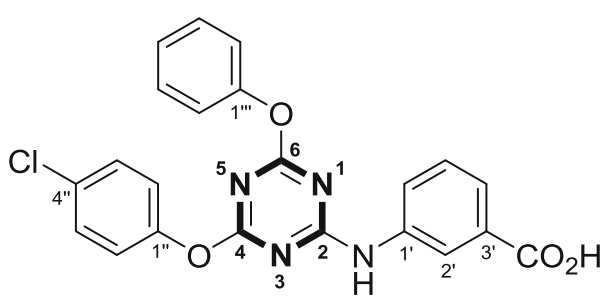

Fig. 4 Explanation of code for peak assignment 
<smiles>CC(=O)c1cccc(Nc2nc(Oc3ccccc3)nc(Oc3ccc(Cl)cc3)n2)c1</smiles>

$5 c$

Fig. 5 Compound $\mathbf{5 c}$, an example for unexpected ${ }^{13} \mathrm{C}$ NMR behavior

intensity signals (Fig. 6 top) or were not discernable as distinct peaks, in which case they emerged only as a small bulge in the spectrum. This is true for the magnetic field strength of the instrument that was used to measure most of the samples $(4.7 \mathrm{~T}$, corresponding to $200 \mathrm{MHz}$ of proton resonance frequency).

In many cases, the total number of carbon signals of the triazine compounds bearing two aryloxy and one phenylamino moiety showed more carbon signals than was expected. What is more, additional peak splitting was observed when the same sample was measured using higher resolution and field strength $(400 \mathrm{MHz}$, compound 5c, Fig. 6 middle vs. top).

Thus, it seemed that in solution different long-lived (on the NMR time scale) conformers were present to give rise to more signals than expected. To test this hypothesis, high-temperature ${ }^{13} \mathrm{C}$ spectra (at $120{ }^{\circ} \mathrm{C}$ ) were recorded. Indeed, under such conditions, fewer carbon signals were observed, confirming that the compound must experience hindered internal rotation at room temperature (Fig. 6, bottom). To elucidate the situation further, the simpler compound 6 was also subjected to ${ }^{13} \mathrm{C}$ NMR-measurement at $120^{\circ} \mathrm{C}$. The effect was particularly prominent in the case of carbon positions $\mathrm{C} 4$ and $\mathrm{C} 6$; they showed up as two broad signals at 171.8 and $172.3 \mathrm{ppm}$ at room temperature, but also coalesced at $171.5 \mathrm{ppm}$ as a sharp peak, making it very plausible that rotation around the $\mathrm{C} 2-\mathrm{N}$ bond was slowed down at room temperature. This can be explained by considering the highly electron-deficient triazine and the appropriate resonance contributor as in Fig. 7. C4 and C6 then adopt different relative conformations, i.e., cis and trans to carbons $\mathrm{C}^{2}$ ' and $\mathrm{C} 6$ ', respectively, as the $\mathrm{C} 2-\mathrm{N}$ bond displays partial double bond character. These conformations are stable long enough that carbon nuclei $\mathrm{C} 4$ and C6 become magnetically different and are visible on the NMR time scale as separate signals.

In addition, if these two carbons are also chemically unequal, the two stable conformers become different rotamers, and the expected two signals split into four (as with 5c, Figs. 6, 8). There is no cis/trans isomerism of this sort regarding carbon $\mathrm{C} 2$ itself, and this nucleus is, therefore, unaffected by peak splitting.

There are no additional peaks in the spectra of compounds where the $\mathrm{NH}$ is replaced by some other atom, such as sulfur in 7. It, therefore, appears evident that the hindered rotation around this carbon-nitrogen bond is responsible for this isomerism as observed with compounds like $\mathbf{5 c}$.

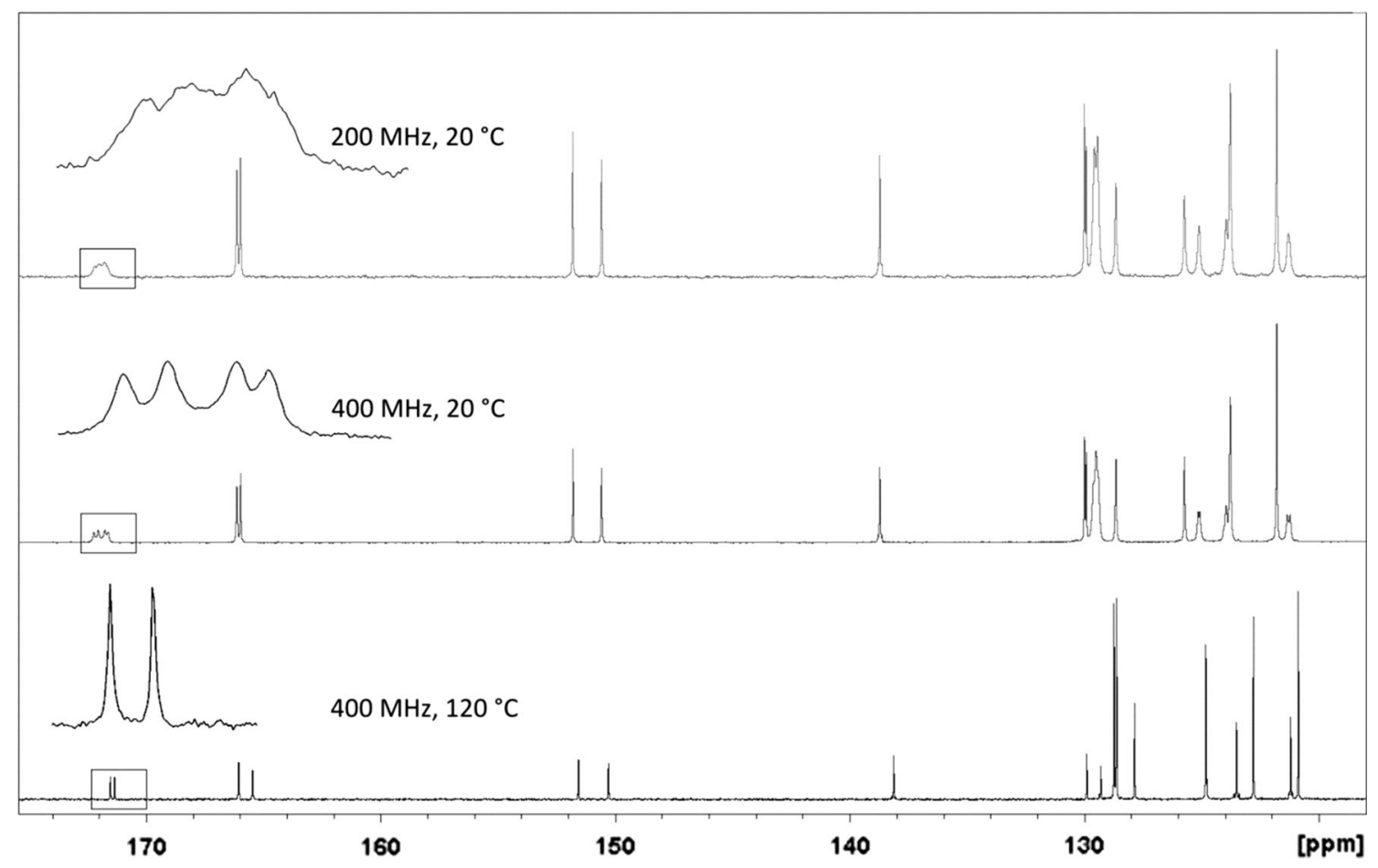

Fig. $6{ }^{13} \mathrm{C}$ NMR of compound $\mathbf{5 c}$, recorded under different conditions 
Fig. 7 Low- versus hightemperature NMR of compound 6<smiles>C[C@@H](O)c1nc(Oc2ccccc2)nc([N+](C)(C)c2ccccc2)n1</smiles>
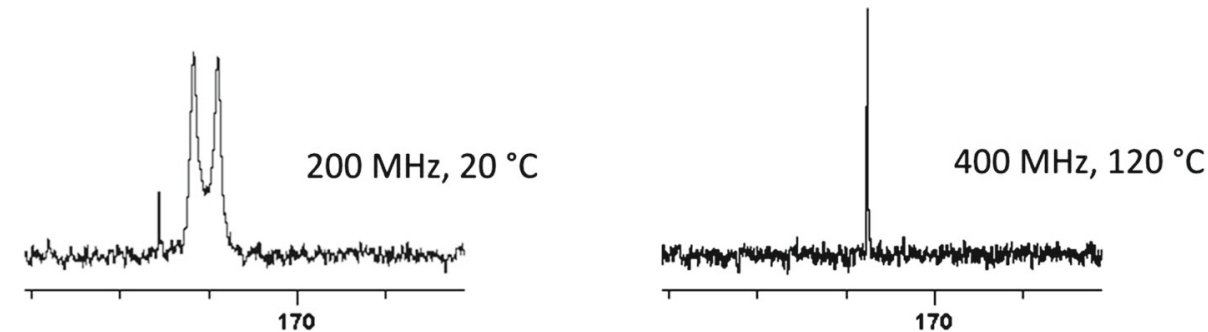<smiles>CC(=O)c1cccc(Nc2nc(Oc3ccccc3)nc(Oc3ccc(Cl)cc3)n2)c1</smiles>

5c

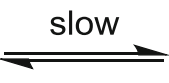<smiles>CC(=O)c1cccc(Nc2nc(Oc3ccccc3)nc(Oc3ccc(Cl)cc3)n2)c1</smiles>

Fig. 8 Rotamers of compound $\mathbf{5 c}$

\section{2,4-Dichloro-6-phenoxy-1,3,5-triazine $\quad\left(2 \mathrm{a}, \quad \mathrm{C}_{9} \mathrm{H}_{5} \mathrm{Cl}_{2} \mathrm{~N}_{3} \mathrm{O}\right)$} 2,4,6-Trichloro-1,3,5-triazine $(1.225 \mathrm{~g}, 6.64 \mathrm{mmol}, 1.00$ equiv.), dissolved in $14 \mathrm{~cm}^{3} \mathrm{THF}$, was cooled to $-15^{\circ} \mathrm{C}$ (ice/salt bath), and then, $0.858 \mathrm{~g}$ DIPEA (6.64 mmol, 1.00 equiv.) and $0.625 \mathrm{~g}$ phenol (6.64 mmol, 1.00 equiv.) were added slowly while stirring. The reaction was kept at that temperature for $30 \mathrm{~min}$, after that the temperature was raised to $0{ }^{\circ} \mathrm{C}$ and stirring was continued for another $3.75 \mathrm{~h}$. Then, the mixture was warmed to r. t. within $30 \mathrm{~min}$. The formed DIPEA-hydrochloride was removed by filtration and washed with additional THF $\left(2 \times 10 \mathrm{~cm}^{3}\right)$. The solution was evaporated to give the solid crude product. It was again dissolved in $100 \mathrm{~cm}^{3}$ EtOAc and this organic solution was washed with saturated $\mathrm{NaHCO}_{3}$ solution $\left(2 \times 100 \mathrm{~cm}^{3}\right), 100 \mathrm{~cm}^{3} 5 \% \mathrm{KHSO}_{4}$, and $100 \mathrm{~cm}^{3}$ brine. After drying over $\mathrm{Na}_{2} \mathrm{SO}_{4}$, the solvent was removed and the product dried in vacuo without further purification. Yield: $1.521 \mathrm{~g}$ (95\%); appearance: colorless solid; m.p.: $115-116^{\circ} \mathrm{C}$ (lit.: $111.5-113.8^{\circ} \mathrm{C}$ ) [52]; $R_{f}=0.50 \quad$ (hexane/EtOAc, 5:1); ${ }^{1} \mathrm{H} \quad \mathrm{NMR} \quad\left(\mathrm{CDCl}_{3}\right.$, $200 \mathrm{MHz}$ ): $\delta=7.13-7.23$ (m, 2H, H2' \& H6'), 7.28-7.39 (m, 1H, H4'), 7.41-7.53 (m, 2H, H3' \& H5') ppm; ${ }^{13} \mathrm{C}$ NMR $\left(\mathrm{CDCl}_{3}, 50 \mathrm{MHz}\right): \delta=121.1$ (d, C2' \& C6'), 127.1 (d, C4'), 130.1 (d, C3' \& C5'), 151.2 (s, C1'), 171.3 (s, C6), 173.2 (s, C2 \& C4) ppm; GC-MS (EI, $70 \mathrm{eV}$ ): $t_{R-}$ $=8.08 \mathrm{~min} ; \mathrm{m} / \mathrm{z}(\%)=243\left(\mathrm{M}^{+}, 10\right), 241\left(\mathrm{M}^{+}, 15\right), 208$ (32), 206 (100), 143 (11).

\section{2,4-Dichloro-6-(4-chlorophenoxy)-1,3,5-triazine}

$\left(2 \mathrm{~b}, \mathrm{C}_{9} \mathrm{H}_{4} \mathrm{Cl}_{3} \mathrm{~N}_{3} \mathrm{O}\right)$ The reaction was performed in an $8 \mathrm{~cm}^{3}$ glass vial, using a cryo or thermo block. 2,4,6-Trichlorotriazine (369 mg, $2.00 \mathrm{mmol}, 1.00$ equiv.) was dissolved in $3.0 \mathrm{~cm}^{3} \mathrm{THF}$ and cooled to $-35^{\circ} \mathrm{C}$. DIPEA $(349 \mathrm{mg}$, $2.70 \mathrm{mmol}$, 1.35 equiv.) was added to $257 \mathrm{mg}$ 4-chlorophenol (2.00 mmol, 1.00 equiv.), dissolved in $1.6 \mathrm{~cm}^{3} \mathrm{THF}$; the mixture was also cooled to $-35^{\circ} \mathrm{C}$, before this solution was added slowly to the trichlorotriazine solution while stirring. To ensure complete transfer, another $1.6 \mathrm{~cm}^{3}$ of $\mathrm{THF}$, cooled to $-35^{\circ} \mathrm{C}$, was used to flush all phenol into the reaction mixture. Stirring was continued at this temperature for $3 \mathrm{~h}$, then at $\mathrm{r}$. t. for $27 \mathrm{~h}$. $\mathrm{H}_{2} \mathrm{O}\left(10 \mathrm{~cm}^{3}\right)$ was then added to the reaction mixture and the compound was extracted with EtOAc $\left(2 \times 10 \mathrm{~cm}^{3}\right)$, 
followed by washing with $10 \mathrm{~cm}^{3}$ brine. The solution was concentrated in vacuo and the compound [53] was purified by column chromatography (MPLC, $90 \mathrm{~g}$ silica, $90 \mathrm{~cm}^{3}$ $\mathrm{min}^{-1}$ flow rate, LP with $2 \%$ EtOAc), and finally dried in vacuo. Yield: $243 \mathrm{mg}$ (44\%); appearance: colorless solid; m.p.: $105-108{ }^{\circ} \mathrm{C} ; R_{f}=0.51$ (hexane/EtOAc, 5:1); ${ }^{1} \mathrm{H}$ NMR $\left(\mathrm{CDCl}_{3}, 200 \mathrm{MHz}\right): \delta=7.03-7.17\left(\mathrm{~m}, 2 \mathrm{H}, \mathrm{H} 2{ }^{\prime} \&\right.$ H6'), 7.32-7.46 (m, 2H, H3' \& H5') ppm; ${ }^{13} \mathrm{C}$ NMR $\left(\mathrm{CDCl}_{3}, 50 \mathrm{MHz}\right): \delta=122.5$ (d, C2' \& C6'), 130.1 (d, C3' \& C5'), 132.6 (s, C4'), 149.5 (s, C1'), 171.0 (s, C6), 173.2 (s, C2 \& C4) ppm; GC-MS (EI, $70 \mathrm{eV}$ ): $t_{R}=9.16 \mathrm{~min} ; \mathrm{m} / \mathrm{z}$ $(\%)=277\left(\mathrm{M}^{+}, 12\right), 275\left(\mathrm{M}^{+}, 15\right), 244(10), 242(62), 241$ (12), 240 (100), 177 (15), 111 (24).

2-Chloro-4,6-diphenoxy-1,3,5-triazine (3a, $\mathrm{C}_{15} \mathrm{H}_{10} \mathrm{CIN}_{3} \mathrm{O}_{2}$ ) 2,4,6-Trichloro-1,3,5-triazine $(1.248 \mathrm{~g}, 6.77 \mathrm{mmol}, 1.00$ equiv.), dissolved in $7 \mathrm{~cm}^{3} \mathrm{THF}$, was cooled to $-30{ }^{\circ} \mathrm{C}$; then, a solution of $0.637 \mathrm{~g}$ phenol $(6.77 \mathrm{mmol}, 1.00$ equiv.) and $0.874 \mathrm{~g}$ DIPEA ( $6.77 \mathrm{mmol}, 1.00$ equiv.) in $7 \mathrm{~cm}^{3}$ THF was added dropwise over 10 min while stirring. The reaction was kept at -35 to $-30{ }^{\circ} \mathrm{C}$ for another $30 \mathrm{~min}$ and subsequently at $0{ }^{\circ} \mathrm{C}$ for $5 \mathrm{~h}$. Then, another solution of $0.637 \mathrm{~g}$ phenol $(6.77 \mathrm{mmol}, 1.00$ equiv.) and $0.874 \mathrm{~g}$ DIPEA (6.77 mmol, 1.00 equiv.) in $7 \mathrm{~cm}^{3}$ THF was added dropwise over $15 \mathrm{~min}$ and the mixture was warmed slowly to $\mathrm{r}$. t. After $48 \mathrm{~h}$, the reaction temperature was raised to $50{ }^{\circ} \mathrm{C}$ and stirring was continued for $4.5 \mathrm{~h}$. The formed DIPEA-hydrochloride was removed by filtration and washed with additional THF $\left(30 \mathrm{~cm}^{3}\right)$. The solution was evaporated to give the solid crude product. It was again dissolved in $100 \mathrm{~cm}^{3}$ EtOAc and this organic solution was washed with $\mathrm{Na}_{2} \mathrm{CO}_{3}$ solution, $0.5 \mathrm{M} \mathrm{HCl}$, as well as brine $\left(2 \times 100 \mathrm{~cm}^{3}\right.$ for each) and subsequently dried over $\mathrm{Na}_{2}$ $\mathrm{SO}_{4}$. The solvent was removed and the crude material was re-crystallized from $n$-hexane/toluene $\left(35 \mathrm{~cm}^{3} / 7 \mathrm{~cm}^{3}\right)$, collected by filtration, washed with $20 \mathrm{~cm}^{3} n$-hexane, and dried in vacuo. Yield: $1.584 \mathrm{~g}(78 \%)$; appearance: colorless crystals; m.p.: $120.5-121{ }^{\circ} \mathrm{C}$ (lit.: $119-121{ }^{\circ} \mathrm{C}$ ) [54]; $R_{f}=0.39 \quad$ (hexane/EtOAc, 5:1); ${ }^{1} \mathrm{H} \quad \mathrm{NMR} \quad\left(\mathrm{CDCl}_{3}\right.$, $200 \mathrm{MHz}$ ): $\delta=7.10-7.19$ (m, 4H, H2' \& H6' \& H2" \& H6”), 7.22-7.32 (m, 2H, H4' \& H4”), 7.34-7.46 (m, 4H, H3' \& H5' \& H3" \& H5”) ppm; ${ }^{13} \mathrm{C}$ NMR $\left(\mathrm{CDCl}_{3}\right.$, $50 \mathrm{MHz}$ ): $\delta=121.2$ (d, C2' \& C6'), 126.5 (d, C4'), 129.7 (d, C3' \& C5'), 151.3 (s, C1'), 172.4 (s, C4 \& C6), 173.8 (s, C2) ppm; GC-MS (EI, $70 \mathrm{eV}$ ): $t_{R}=11.45 \mathrm{~min} ; \mathrm{m} / z$ $(\%)=301\left(\mathrm{M}^{+}, 11\right), 300\left(\mathrm{M}^{+}, 10\right), 299\left(\mathrm{M}^{+}, 33\right), 298$ (16), 264 (57), 208 (32), 207 (11), 206 (100), 180 (12), 145 (15), 140 (10), 138 (31), 121 (20).

\section{Synthesis of (4,6-disubstituted-1,3,5-triazin-2- yl)aminobenzoic acids $4 a-4 k-$ general procedure $A$}

The reaction was performed in an $8 \mathrm{~cm}^{3}$ glass vial, using a thermo block. Formic acid $\left(4.0 \mathrm{~cm}^{3}\right)$ was added to 1,1 dimethylethyl aminobenzoate (1.00 equiv.). This mixture was briefly sonicated until the starting material was dissolved and stirred at the specified temperature until TLC showed complete conversion. The content of the reaction vial was transferred to a round bottom flask and the vial was flushed with a few $\mathrm{cm}^{3}$ of $\mathrm{CH}_{2} \mathrm{Cl}_{2}$. Then, the solvents were removed under reduced pressure, $10 \mathrm{~cm}^{3} \mathrm{Et}_{2} \mathrm{O}$ was added to facilitate the removal of residual formic acid, the solution evaporated again and finally dried in vacuo.

3-[(4,6-Diphenoxy-1,3,5-triazin-2-yl)amino]benzoic acid (4a, $\mathrm{C}_{22} \mathrm{H}_{16} \mathrm{~N}_{4} \mathrm{O}_{4}$ ) Prepared according to general procedure $A$ using $46 \mathrm{mg}$ 1,1-dimethylethyl aminobenzoate $\mathbf{8 a}$ ( $0.10 \mathrm{mmol}, 1.00$ equiv.); $50{ }^{\circ} \mathrm{C}$ for $1 \mathrm{~h}$; compound $4 \mathrm{a}$ is literature-known [31, 55]. Yield: $40 \mathrm{mg}$ (quant.); appearance: off-white solid; m.p.: $267.5-270{ }^{\circ} \mathrm{C} ; R_{f}=0.57$ (hexane/EtOH, 1:1); ${ }^{1} \mathrm{H}$ NMR (DMSO- $d_{6}, 200 \mathrm{MHz}$ ): $\delta=7.14-7.35(\mathrm{~m}, 7 \mathrm{H}), 7.38-7.52(\mathrm{~m}, 4 \mathrm{H}), 7.56(\mathrm{~d}$, $\left.J_{3}=7.7 \mathrm{~Hz}, 1 \mathrm{H}\right), 7.76\left(\mathrm{~d}, J_{3}=8.3 \mathrm{~Hz}, 1 \mathrm{H}\right), 8.03(\mathrm{~s}, 1 \mathrm{H}$, $\left.\mathrm{H} 2^{\prime}\right), 10.40$ (s, 1H, NH), $12.98\left(\mathrm{bs}, 1 \mathrm{H}, \mathrm{CO}_{2} \mathrm{H}\right) \mathrm{ppm} ;{ }^{13} \mathrm{C}$ NMR (DMSO- $\left.d_{6}, 50 \mathrm{MHz}\right): \delta=121.5\left(\mathrm{~d}, \mathrm{C} 2^{\prime}\right), 121.8(\mathrm{~d}$, C2" \& C2'” \& C6" \& C6"”), 124.1 (d, C4'), 124.8 (d, C6'), 125.7 (d, C4" \& C4'”), 128.5 (d, C5'), 129.6 (d, C3" \& C3" " \& C5" \& C5'”), 131.2 (s, C3'), 138.6 (s, C1'), 151.8 (s, C1", C1"”), 166.2 (s, C2), 167.1 (s, $\mathrm{CO}_{2}$ ), 171.8 \& 172.2 (bs, C4 \& C6, rotamers) ppm.

3-[[4-(4-Chlorophenoxy)-6-phenoxy-1,3,5-triazin-2-

yl]amino]benzoic acid (4b, $\left.\mathrm{C}_{22} \mathrm{H}_{15} \mathrm{CIN}_{4} \mathrm{O}_{4}\right)$ Prepared according to general procedure A using $49 \mathrm{mg}$ 1,1-dimethylethyl aminobenzoate $\mathbf{8 b}(0.10 \mathrm{mmol}, 1.00$ equiv.); $50{ }^{\circ} \mathrm{C}$ for $1 \mathrm{~h}$. Yield: $44 \mathrm{mg}$ (quant.); appearance: beige solid; m.p.: $244-247{ }^{\circ} \mathrm{C} ; R_{f}=0.63$ (hexane/EtOH, $1: 1$ ); ${ }^{1} \mathrm{H}$ NMR (DMSO- $\left.d_{6}, 200 \mathrm{MHz}\right): \delta=7.14-7.38(\mathrm{~m}, 6 \mathrm{H})$, 7.38-7.63 (m, 5H), $7.72\left(\mathrm{~d}, J_{3}=8.4 \mathrm{~Hz}, 1 \mathrm{H}\right), 8.04(\mathrm{~s}, 1 \mathrm{H}$, $\mathrm{H} 2^{\prime}$ ), 10.42 (s, $\left.1 \mathrm{H}, \mathrm{NH}\right), 12.91$ (bs, $\left.1 \mathrm{H}, \mathrm{CO}_{2} \mathrm{H}\right) \mathrm{ppm} ;{ }^{13} \mathrm{C}$ NMR (DMSO- $\left.d_{6}, 50 \mathrm{MHz}\right): \delta=121.5\left(\mathrm{~d}, \mathrm{C} 2{ }^{\prime}\right), 121.8(\mathrm{~d}$, C2" " \& C6" "), 123.8 (d, C2" \& C6"), 124.2 (d, C4'), 124.8 (d, C6'), 125.8 (d, C4'”), 128.5 (d, C5'), 129.5 (d, C3"** \& C5”*), 129.6 (d, C3'”* \& C5'”*), 129.9 (s, C4”), 131.2 (s, C3'), 138.5 (s, C1'), 150.6 (s, C1"), 151.8 (s, C1'”), 166.1 (s, C2), $167.0\left(\mathrm{~s}, \mathrm{CO}_{2}\right), 171.3-172.4$ (C4 \& C6) ppm.

\section{3-[[4-(3-Chlorophenoxy)-6-phenoxy-1,3,5-triazin-2-}

yl]amino]benzoic acid $\left(4 \mathrm{c}, \quad \mathrm{C}_{22} \mathrm{H}_{15} \mathrm{ClN}_{4} \mathrm{O}_{4}\right)$ Prepared according to general procedure A using $49 \mathrm{mg}$ 1,1-dimethylethyl aminobenzoate $8 \mathbf{c}(0.10 \mathrm{mmol}, 1.00$ equiv.); $50{ }^{\circ} \mathrm{C}$ for $1 \mathrm{~h}$. Yield: $44 \mathrm{mg}$ (quant.); appearance: off-white 
solid; m.p.: $227-230{ }^{\circ} \mathrm{C} ; R_{f}=0.59$ (hexane/EtOH, $\left.1: 1\right) ;{ }^{1} \mathrm{H}$ NMR (DMSO- $\left.d_{6}, 200 \mathrm{MHz}\right): \delta=7.16-7.53(\mathrm{~m}, 10 \mathrm{H})$, $7.58\left(\mathrm{~d}, J_{3}=7.7 \mathrm{~Hz}, 1 \mathrm{H}\right), 7.75\left(\mathrm{~d}, J_{3}=7.9 \mathrm{~Hz}, 1 \mathrm{H}\right), 8.04$ (s, $\left.1 \mathrm{H}, \mathrm{H} 2^{\prime}\right), 10.45(\mathrm{~s}, 1 \mathrm{H}, \mathrm{NH}), 12.97\left(\mathrm{bs}, 1 \mathrm{H}, \mathrm{CO}_{2} \mathrm{H}\right)$ ppm; ${ }^{13} \mathrm{C}$ NMR (DMSO- $d_{6}, 50 \mathrm{MHz}$ ): $\delta=120.8$ (d, C6"), 121.5 (d, C2'), 121.8 (d, C2'” \& C6'”), 122.4 (d, C2"), 124.3 (d, C4'), 124.8 (d, C6'), 125.8 (d, C4'”), 125.9 (d, C4"), 128.5 (d, C5'), 129.6 (d, C3'” \& C5'”), 131.0 (d, C5”), 131.4 (s, C3'), 133.4 (s, C3"), 138.5 (s, C1'), 151.8 (s, C1'”), 152.4 (s, C1"), 166.2 (s, C2), 167.1 (s, $\left.\mathrm{CO}_{2}{ }^{*}\right)$, 171.3-172.3 (C4 \& C6, not resolved) ppm.

\section{3-[[4-(2-Chlorophenoxy)-6-phenoxy-1,3,5-triazin-2-}

\section{yl]amino]benzoic acid $\left(4 \mathrm{~d}, \quad \mathrm{C}_{22} \mathrm{H}_{15} \mathrm{ClN}_{4} \mathrm{O}_{4}\right)$ Prepared} according to general procedure $\mathrm{A}$ using $49 \mathrm{mg}$ 1,1-dimethylethyl aminobenzoate $8 d$ ( $0.10 \mathrm{mmol}, 1.00$ equiv.); $50{ }^{\circ} \mathrm{C}$ for $1 \mathrm{~h}$. Yield: $44 \mathrm{mg}$ (quant.); appearance: colorless crystals; m.p.: $249.5-251.5^{\circ} \mathrm{C} ; R_{f}=0.60$ (hexane/EtOH, $1: 1$ ); ${ }^{1} \mathrm{H}$ NMR (DMSO- $\left.d_{6}, 200 \mathrm{MHz}\right): \delta=7.13-7.51$ (m, 9H), 7.52-7.75 (m, 3H), 7.99 (s, 1H, H2'), 10.49 (s, 1H, $\mathrm{NH}), 12.93$ (bs, $\left.1 \mathrm{H}, \mathrm{CO}_{2} \mathrm{H}\right) \mathrm{ppm} ;{ }^{13} \mathrm{C}$ NMR (DMSO- $d_{6}$, $50 \mathrm{MHz}$ ): $\delta=121.6$ (d, C2'), 121.8 (d, C2'” \& C6'”), 124.3 (d, C6"*), 124.8 (d, C6'), 125.8 (d, C4'”), 126.0 (s, C2"), 127.5 (d, C4"*), 128.5 (d, C5”*), 128.6 (d, C5'*), 129.7 (d, C3"” \& C5"”), 130.3 (d, C3"), 131.3 (s, C3'), 138.5 (s, C1'), 147.7 (s, C1"), 151.8 (s, C1'”), 166.2 (s, $\mathrm{C} 2$ ), 167.1 (s, $\mathrm{CO}_{2}$ ), 171.2-172.5 (C4 \& C6, not resolved) $\mathrm{ppm}$, one d signal missing possibly due to overlap.

\section{3-[[4-[4-(1,1-Dimethylethyl)phenoxy]-6-phenoxy-1,3,5-tri-} azin-2-yl]amino]benzoic acid (4e, $\left.\mathrm{C}_{26} \mathrm{H}_{24} \mathrm{~N}_{4} \mathrm{O}_{4}\right)$ Prepared according to general procedure A using $51 \mathrm{mg}$ 1,1-dimethylethyl aminobenzoate 8e $(0.10 \mathrm{mmol}, 1.00$ equiv.); $45^{\circ} \mathrm{C}$ for $1 \mathrm{~h}$. Yield: $46 \mathrm{mg}$ (quant.); appearance: off-white solid; m.p.: $245-247{ }^{\circ} \mathrm{C} ; R_{f}=0.67$ (hexane/EtOH, 1:1); ${ }^{1} \mathrm{H}$ NMR (DMSO- $\left.d_{6}, 200 \mathrm{MHz}\right): \delta=1.30\left(\mathrm{~s}, 9 \mathrm{H},\left(\mathrm{CH}_{3}\right)_{3}\right)$, 7.10-7.33 (m, 6H), 7.37-7.51 (m, 4H), $7.57 \quad(\mathrm{~d}$, $\left.J_{3}=7.7 \mathrm{~Hz}, 1 \mathrm{H}\right), 7.75\left(\mathrm{~d}, J_{3}=8.7 \mathrm{~Hz}, 1 \mathrm{H}\right), 8.05(\mathrm{~s}, 1 \mathrm{H}$, H2'), 10.40 (s, 1H, NH), 12.92 (bs, $\left.1 \mathrm{H}, \mathrm{CO}_{2} \mathrm{H}\right) \mathrm{ppm} ;{ }^{13} \mathrm{C}$ NMR (DMSO- $\left.d_{6}, 50 \mathrm{MHz}\right): \delta=31.3\left(\mathrm{q}, \mathrm{C}\left(\mathrm{CH}_{3}\right)_{3}\right), 34.3$ (s, $\left.\mathrm{C}\left(\mathrm{CH}_{3}\right)_{3}\right), 121.2$ (d, $\mathrm{C} 2$ " \& C6"), 121.6 (d, C2'), 121.8 (d, C2'” \& C6'”), 124.1 (d, C4'), 124.8 (d, C6'), 125.7 (d, C4'”), 126.3 (d, C3" \& C5"), 128.4 (d, C5'), 129.6 (d, C3'” \& C5'”), 131.2 (s, C3'), 138.7 (s, C1'), 148.0 (s, C4"*), 149.5 (s, C1"*), 151.8 (s, C1'”), 166.2 (s, C2), 167.1 (s, $\mathrm{CO}_{2}$ ), 171.6-172.7 (C4 \& C6, not resolved) ppm.

\section{3-[[4-(4-Methoxyphenoxy)-6-phenoxy-1,3,5-triazin-2-}

yl]amino]benzoic acid (4f, $\mathrm{C}_{23} \mathrm{H}_{18} \mathrm{~N}_{4} \mathrm{O}_{5}$ ) Prepared according to general procedure A using $49 \mathrm{mg}$ 1,1-dimethylethyl aminobenzoate $8 \mathbf{8 f}(0.10 \mathrm{mmol}, 1.00$ equiv. $) ; 50{ }^{\circ} \mathrm{C}$ for $1 \mathrm{~h}$. Yield: $43 \mathrm{mg}$ (quant.); appearance: off-white solid; m.p.: 233-236.5 ${ }^{\circ} \mathrm{C} ; R_{f}=0.65$ (hexane/EtOH, 1:1); ${ }^{1} \mathrm{H}$ NMR (DMSO- $\left.d_{6}, 200 \mathrm{MHz}\right): \delta=3.77\left(\mathrm{~s}, 3 \mathrm{H}, \mathrm{OCH}_{3}\right), 6.97$ (d,
$J_{3}=9.0 \mathrm{~Hz}, 2 \mathrm{H}, \mathrm{H} 3 "$ \& H5"), 7.13-7.34 (m, 6H), $7.39-7.51(\mathrm{~m}, 2 \mathrm{H}), 7.56\left(\mathrm{~d}, J_{3}=7.6 \mathrm{~Hz}, 1 \mathrm{H}\right), 7.74(\mathrm{~d}$, $\left.J_{3}=8.2 \mathrm{~Hz}, 1 \mathrm{H}\right), 8.04(\mathrm{~s}, 1 \mathrm{H}, \mathrm{H} 2$ '), $10.37(\mathrm{~s}, 1 \mathrm{H}, \mathrm{NH})$, 12.93 (bs, $1 \mathrm{H}, \quad \mathrm{CO}_{2} \mathrm{H}$ ) ppm; ${ }^{13} \mathrm{C}$ NMR (DMSO- $d_{6}$, $50 \mathrm{MHz}): \delta=55.5$ (q, $\left.\mathrm{OCH}_{3}\right), 114.5$ (d, C3" \& C5"), 121.5 (d, C2'), 121.8 (d, C2'” \& C6'”), 122.7 (d, C2" \& C6”), 124.1 (d, C4'), 124.8 (d, C6'), 125.7 (d, C4'”), 128.5 (d, C5'), 129.6 (d, C3'” \& C5'”), 131.3 (s, C3'), 138.6 (s, C1'), 145.2 (s, C1"), 151.8 (s, C1'”), 156.9 (s, C4”), 166.2 (s, C2), 167.1 (s, $\left.\mathrm{CO}_{2}\right), 171.3-172.7$ (C4 \& C6, not resolved) ppm.

\section{3-[[4-(4-Cyanophenoxy)-6-phenoxy-1,3,5-triazin-2-}

yl]amino]benzoic acid $\left(4 \mathrm{~g}, \mathrm{C}_{23} \mathrm{H}_{15} \mathrm{~N}_{5} \mathrm{O}_{4}\right)$ Prepared according to general procedure $\mathrm{A}$ using $48 \mathrm{mg}$ 1,1-dimethylethyl aminobenzoate $8 \mathrm{~g}(0.10 \mathrm{mmol}, 1.00$ equiv. $) ; 45^{\circ} \mathrm{C}$ for $1 \mathrm{~h}$. Yield: $43 \mathrm{mg}$ (quant.); appearance: colorless solid; m.p.: $150{ }^{\circ} \mathrm{C}$ (decomp.); $R_{f}=0.65$ (hexane/EtOH, $1: 1$ ); ${ }^{1} \mathrm{H}$ NMR (DMSO- $\left.d_{6}, 200 \mathrm{MHz}\right): \delta=7.16-7.35(\mathrm{~m}, 4 \mathrm{H}), 7.37-7.77$ (m, 6H), $7.94\left(\mathrm{~d}, J_{3}=8.1 \mathrm{~Hz}, 2 \mathrm{H}, \mathrm{H} 3\right.$ " \& H5"), 8.06 (s, $1 \mathrm{H}, \mathrm{H} 2$ ') 10.48 (s, $1 \mathrm{H}, \mathrm{NH}) \mathrm{ppm}, \mathrm{CO}_{2} \mathrm{H}$ not visible; ${ }^{13} \mathrm{C}$ NMR (DMSO- $d_{6}, 50 \mathrm{MHz}$ ): $\delta=108.6$ (s, C4"), 118.5 (s, CN), 121.66 (d, C2'), 121.76 (d, C2'” \& C6'”), 123.3 (d, C2" \& C6"), 124.4 (d, C4'), 124.8 (d, C6'), 125.8 (d, C4'”), 128.5 (d, C5'), 129.6 (d, C3'" \& C5'”), 131.6 (s, C3'), 134.0 (d, C3" \& C5"), 138.4 (s, C1'), 151.6 (s, C1'”), 155.2 (s, C1"), 166.2 (s, C2), 167.1 (s, $\mathrm{CO}_{2}$ ), 170.9-172.2 (C4 \& C6, not resolved) ppm.

\section{3-[[4-(3-Nitrophenoxy)-6-phenoxy-1,3,5-triazin-2-}

yl]amino]benzoic acid $\left(4 \mathrm{~h}, \mathrm{C}_{22} \mathrm{H}_{15} \mathrm{~N}_{5} \mathrm{O}_{6}\right)$ Prepared according to general procedure $\mathrm{A}$ using $40 \mathrm{mg}$ 1,1-dimethylethyl aminobenzoate $8 \mathrm{~h}(0.08 \mathrm{mmol}, 1.00$ equiv. $) ; 45^{\circ} \mathrm{C}$ for $1 \mathrm{~h}$. Yield: $36 \mathrm{mg}$ (quant.); appearance: beige solid; m.p.: $225-228{ }^{\circ} \mathrm{C} ; \quad R_{f}=0.51 \quad$ (hexane/EtOH, $1: 1$ ); ${ }^{1} \mathrm{H}$ NMR (DMSO- $\left.d_{6}, 200 \mathrm{MHz}\right): \delta=7.16-7.33(\mathrm{~m}, 4 \mathrm{H}), 7.34-7.50$ $(\mathrm{m}, 2 \mathrm{H}), 7.57\left(\mathrm{~d}, J_{3}=7.7 \mathrm{~Hz}, 1 \mathrm{H}\right), 7.63-7.84(\mathrm{~m}, 3 \mathrm{H})$, 8.06 (s, 1H, H2'), 8.15 (d, $\left.J_{3}=7.5 \mathrm{~Hz}, 1 \mathrm{H}\right), 8.22(\mathrm{~m}, 1 \mathrm{H}$, $\mathrm{H} 2$ "), 10.49 (s, 1H, NH), 12.83 (bs, $\left.1 \mathrm{H}, \mathrm{CO}_{2} \mathrm{H}\right) \mathrm{ppm} ;{ }^{13} \mathrm{C}$ NMR (DMSO- $d_{6}, 50 \mathrm{MHz}$ ): $\delta=117.5$ (d, C2”), 120.7 (d, C4"), 121.5 (d, C2'), 121.8 (d, C2'" \& C6"”), 124.4 (d, C4'), 124.8 ((d, C6'), 125.8 (d, C4'”), 128.5 (d, C6"*), 128.9 (d, C5'*), 129.6 (d, C3'” \& C5'”), 130.8 (d, C5”), 131.3 (s, C3'), 138.5 (s, C1'), 148.4 (s, C3"), 151.8 (s, $\left.\mathrm{C} 1{ }^{\prime}{ }^{*}\right), 152.0$ (s, C1"*), 166.2 (s, C2), $167.1\left(\mathrm{~s}, \mathrm{CO}_{2}\right)$, 171.2-172.5 (C4 \& C6, not resolved) ppm.

3-[[4-(4-Formyl-2-methoxyphenoxy)-6-phenoxy-1,3,5-triazin-2-yl]amino]benzoic acid $\left(4 \mathrm{i}, \mathrm{C}_{24} \mathrm{H}_{18} \mathrm{~N}_{4} \mathrm{O}_{6}\right)$ Prepared according to general procedure A using $39 \mathrm{mg}$ 1,1-dimethylethyl aminobenzoate $8 \mathbf{i}$ ( $0.075 \mathrm{mmol}, 1.00$ equiv.); $45{ }^{\circ} \mathrm{C}$ for $1 \mathrm{~h}$. Yield: $34 \mathrm{mg}$ (quant.); appearance: pale yellow solid; m.p.: $217.5-220{ }^{\circ} \mathrm{C} ; R_{f}=0.62$ (hexane/ EtOH, $1: 1$ ); ${ }^{1} \mathrm{H}$ NMR (DMSO- $d_{6}, 200 \mathrm{MHz}$ ): $\delta=3.86$ (s, 
$\left.3 \mathrm{H}, \mathrm{OCH}_{3}\right), 7.12-7.33(\mathrm{~m}, 4 \mathrm{H}), 7.35-7.75(\mathrm{~m}, 7 \mathrm{H}), 8.00(\mathrm{~s}$, 1H, H2'), 10.00 (s, 1H, CHO), 10.44 (s, 1H, NH) ppm, $\mathrm{CO}_{2} \mathrm{H}$ not visible; ${ }^{13} \mathrm{C}$ NMR (DMSO- $d_{6}, 50 \mathrm{MHz}$ ): $\delta=56.1$ (q, $\left.\mathrm{OCH}_{3}\right), 112.3$ (d, C3"), 121.5 (d, C2'), 121.8 (d, C2'" \& C6"”), 123.7 (d, C5"*), 123.8 (d, C4'*), 124.3 (d, C6"*), 124.7 (d, C6'), 125.8 (d, C4'”), 128.5 (d, C5'), 129.7 (d, C3"” \& C5" "), 131.4 (s, C3'), 135.1 (s, C4"), 138.5 (s, C1'), 145.3 (s, C1"*), 151.70 (s, C2”*), 151.75 (s, C1 $\left.{ }^{\prime}, *\right), 166.1$ (s, C2), 167.1 (s, $\left.\mathrm{CO}_{2}^{*}\right), 171.2-172.7$ (C4 \& C6, not resolved), 192.1 (d, CHO) ppm.

\section{3-[[4-(4-Formyl-2-methoxyphenoxy)-6-(4-methoxyphe-} noxy)-1,3,5-triazin-2-yl]amino]benzoic acid (4j, $\mathrm{C}_{25} \mathrm{H}_{20} \mathrm{~N}_{4} \mathrm{O}_{7}$ ) Prepared according to general procedure A using $37 \mathrm{mg}$ 1,1-dimethylethyl aminobenzoate $8 \mathbf{j}$ (0.068 mmol, 1.00 equiv.); $45^{\circ} \mathrm{C}$ for $1.25 \mathrm{~h}$. Yield: $33 \mathrm{mg}$ (quant.); appearance: colorless solid; m.p.: $180-182{ }^{\circ} \mathrm{C} ; R_{f}=0.59$ (hexane/ EtOH, 1:1); ${ }^{1} \mathrm{H}$ NMR (DMSO- $d_{6}, 200 \mathrm{MHz}$ ): $\delta=3.76$ (s, $3 \mathrm{H}, \mathrm{C} 4$ " ' $\left.\mathrm{OCH}_{3}\right), 3.86$ (s, 3H, C2" $\left.\mathrm{OCH}_{3}\right), 6.96$ (d, $J_{3}=9.1 \mathrm{~Hz}, 2 \mathrm{H}, \mathrm{H} 3$ " " \& $\mathrm{H} 5$ '"), 7.11-7.29 (m, 3H), 7.42-7.83 (m, 5H), 8.02 (s, 1H, H2'), 10.00 (s, 1H, CHO), 10.42 (s, 1H, NH), 12.92 (bs, $\left.1 \mathrm{H}, \mathrm{CO}_{2} \mathrm{H}\right) \mathrm{ppm} ;{ }^{13} \mathrm{C} \mathrm{NMR}$ $\left(\mathrm{DMSO}-d_{6}, 100 \mathrm{MHz}\right): \delta=55.9$ (q, C4' $\left.{ }^{\prime} \mathrm{OCH}_{3}\right), 56.5$ (q, $\mathrm{C}^{2}{ }^{\prime} \mathrm{OCH}_{3}$ ), 112.8 (d, C3"), 114.9 (d, C3" " \& C5" "), 122.4 (d), 123.1 (d, C2" " \& C6" "), 124.1 (d), 125.0 (d), 127.6 (d), 135.4 (s, C4”), 137.9 (s, C1'), 139.7 (s), 145.7 (s, C1”*), 145.8 (s, C1" "*), 152.2 (s, C2"), 157.3 (s, C4" "), 166.6 (s), 171.4-173.0 (C4 \& C6, not resolved), 192.6 (d, CHO) ppm, one $\mathrm{s}$ and two $\mathrm{d}$ signals missing possibly due to overlap.

\section{3-[[4-(4-Cyanophenoxy)-6-(4-methoxyphenoxy)-1,3,5-tri-} azin-2-yl]amino]benzoic acid $\left(4 \mathrm{k}, \mathrm{C}_{24} \mathrm{H}_{17} \mathrm{~N}_{5} \mathrm{O}_{5}\right)$ Prepared according to general procedure A using $31 \mathrm{mg}$ 1,1-dimethylethyl aminobenzoate $\mathbf{8 k}$ (0.06 mmol, 1.00 equiv.); $45^{\circ} \mathrm{C}$ for $1 \mathrm{~h}$. Yield: $27 \mathrm{mg}$ (quant.); appearance: colorless solid; m.p.: $240.5-242.5{ }^{\circ} \mathrm{C} ; R_{f}=0.48$ (hexane/EtOH, $1: 1)$; ${ }^{1} \mathrm{H}$ NMR (DMSO- $\left.d_{6}, 200 \mathrm{MHz}\right): \delta=3.77(\mathrm{~s}, 3 \mathrm{H}$, $\left.\mathrm{OCH}_{3}\right), 6.97$ (d, $J_{3}=9.0 \mathrm{~Hz}, 2 \mathrm{H}, \mathrm{H} 3$ ' " \& H5 '”), 7.11-7.33 (m, 3H), 7.46-7.80 (m, 4H), 7.94 (d, $J_{3}=8.3 \mathrm{~Hz}, 2 \mathrm{H}, \mathrm{H} 3$ " \& H5"), 8.06 (s, 1H, H2'), 10.44 (s, 1H, NH) ppm, $\mathrm{CO}_{2} \mathrm{H}$ not visible; ${ }^{13} \mathrm{C}$ NMR (DMSO- $d_{6}, 50 \mathrm{MHz}$ ): $\delta=55.5$ (q, $\mathrm{OCH}_{3}$ ), 108.6 (s, C4"), 114.5 (d, C3" " \& C5'”), 118.5 (s, CN), 121.7 (d, C2'), 122.6 (d, C2' " \& C6" '), 123.3 (d, C2" \& C6”), 124.4 (d, C4'), 124.9 (d, C6'), 128.6 (d, C5'), 131.4 (s, C3'), 134.2 (d, C3" \& C5”), 138.5 (s, C1'), 145.2 (s, C1'”), 155.2 (s, C1"), 156.9 (s, C4"”), 166.2 (s, C2), $167.1\left(\mathrm{~s}, \mathrm{CO}_{2}\right)$ ppm, C4 \& $\mathrm{C} 6$ not visible.

\section{Synthesis of methyl and ethyl (4,6-disubstituted- 1,3,5-triazin-2-yl)aminobenzoates-general procedure $B$}

The reaction was performed in an $8 \mathrm{~cm}^{3}$ glass vial, using a cryo or thermoblock. DIPEA (1.10 equiv.) was added to the phenolic compound $\mathrm{R}^{1} \mathrm{PhOH}$ (1.00 equiv.) dissolved in $0.5 \mathrm{~cm}^{3}$ THF. This solution was cooled to $-35^{\circ} \mathrm{C}$ and then added dropwise to a solution of 2,4,6-trichlorotriazine (1.00 equiv.) in $2.0 \mathrm{~cm}^{3} \mathrm{THF}$ at $-35^{\circ} \mathrm{C}$ while stirring. To ensure complete transfer, another $0.5 \mathrm{~cm}^{3}$ of THF was used to flush all phenolic compound into the reaction mixture. Stirring was continued (step 1) at this temperature until TLC indicated complete conversion or no significant change in reaction composition. Then, DIPEA (1.60 equiv.) was added to a solution of phenol $\mathrm{R}^{2} \mathrm{PhOH}$ (1.00 equiv.) in $0.5 \mathrm{~cm}^{3} \mathrm{THF}$, and this mixture was added to the reaction. To ensure complete transfer, another $0.5 \mathrm{~cm}^{3}$ of THF was used to flush all phenolic compounds into the reaction mixture. Stirring was continued (step 2) and checked by TLC. Then, DIPEA (1.50 equiv.), followed by alkyl aminobenzoate (1.15 equiv.), was added directly to the reaction mixture. The reaction was then continued (step 3 ) and checked by TLC. For work-up, $15 \mathrm{~cm}^{3} \mathrm{CH}_{2} \mathrm{Cl}_{2}$ was added to the reaction mixture followed by $15 \mathrm{~cm}^{3} 1 \mathrm{~N}$ $\mathrm{HCl}_{\mathrm{aq}}$, and the compound was extracted. The layers were separated; the organic phase was washed with water $\left(2 \times 15 \mathrm{~cm}^{3}\right)$ and then concentrated in vacuo. Further work-up and purification procedures are given at the respective examples.

Ethyl 4-[(4,6-diphenoxy-1,3,5-triazin-2-yl)amino]benzoate $\left(5 \mathrm{a}, \mathrm{C}_{24} \mathrm{H}_{20} \mathrm{~N}_{4} \mathrm{O}_{4}\right) \quad$ Prepared according to general procedure B using for step 1: $277 \mathrm{mg}$ 2,4,6-trichlorotriazine (1.50 mmol, 1.00 equiv.), $141 \mathrm{mg}$ phenol (1.50 mmol, 1.00 equiv.), $213 \mathrm{mg}$ DIPEA (1.65 mmol, 1.10 equiv.); $-35^{\circ} \mathrm{C}$ for $6 \mathrm{~h}$; step 2: $141 \mathrm{mg}$ phenol (1.50 mmol, 1.00 equiv.), $310 \mathrm{mg}$ DIPEA (2.40 mmol, 1.60 equiv.); $35^{\circ} \mathrm{C}$ for $46 \mathrm{~h}$; step 3: $285 \mathrm{mg}$ ethyl 4-aminobenzoate (1.73 mmol, 1.15 equiv.), $291 \mathrm{mg}$ DIPEA (2.25 mmol, 1.50 equiv.); $40{ }^{\circ} \mathrm{C}$ for $42 \mathrm{~h}$. After the work-up procedure described in general procedure B, $1 \mathrm{~cm}^{3}$ diethyl ether and $2 \mathrm{~cm}^{3} \mathrm{LP}$ were added to induce precipitation and facilitate evaporation to dryness. The crude material was dissolved in a mixture of refluxing $\mathrm{CHCl}_{3} / \mathrm{LP}$ (1: $2,12 \mathrm{~cm}^{3}$ ) and $8 \mathrm{~cm}^{3} n$-hexane was added. After crystallization overnight, the compound was isolated by filtration, washed with $40 \mathrm{~cm}^{3} \mathrm{LP}$, and dried in vacuo. Yield: $611 \mathrm{mg}$ (95\%); appearance: colorless solid; m.p.: $159-161{ }^{\circ} \mathrm{C} ; R_{f}=0.54$ (hexane/EtOAc, 2:1); ${ }^{1} \mathrm{H} \quad \mathrm{NMR} \quad\left(\mathrm{DMSO}-d_{6}, 200 \mathrm{MHz}\right): \delta=1.28 \quad(\mathrm{t}$, $\left.J_{3}=7.1 \mathrm{~Hz}, 3 \mathrm{H}, \mathrm{CH}_{3}\right), 4.25\left(\mathrm{q}, J_{3}=7.1 \mathrm{~Hz}, 2 \mathrm{H}, \mathrm{CH}_{2}\right.$ ), 7.26-7.35 (m, 6H), 7.45-7.57 (m, 6H), 7.63-7.74 (m, 2H), 10.57 (s, 1H, NH) ppm; ${ }^{13} \mathrm{C}$ NMR (DMSO- $d_{6}, 50 \mathrm{MHz}$ ): 
$\delta=14.1\left(\mathrm{q}, \mathrm{CH}_{3}\right), 60.4\left(\mathrm{t}, \mathrm{CH}_{2}\right), 119.4\left(\mathrm{~d}, \mathrm{C} 2{ }^{\prime} \& \mathrm{C}^{\prime}\right.$ '), 121.9 (d, C2" \& C2"” \& C6" \& C6'”), 123.9 (s, C4'), 125.8 (d, C4" \& C4'”), 129.60 (C3' \& C5'), 129.63 (d, C3" \& C3" " \& C5" \& C5" "), 143.0 (s, C1'), 151.9 (s, C1" \& $\mathrm{C} 1$ '”), 165.3 (s, $\mathrm{CO}_{2}$ ), 166.0 (s, C2), 172.1 (s, C4 \& C6, no rotamers visible individually) $\mathrm{ppm}$.

Methyl 3-[(4,6-diphenoxy-1,3,5-triazin-2-yl)amino]benzoate $\left(5 \mathrm{~b}, \mathrm{C}_{23} \mathrm{H}_{18} \mathrm{~N}_{4} \mathrm{O}_{4}\right) \quad$ Prepared according to general procedure B using for step 1: $277 \mathrm{mg}$ 2,4,6-trichlorotriazine (1.50 mmol, 1.00 equiv.), $141 \mathrm{mg}$ phenol ( $1.50 \mathrm{mmol}, 1.00$ equiv.), $213 \mathrm{mg}$ DIPEA (1.65 mmol, 1.10 equiv.); $-35{ }^{\circ} \mathrm{C}$ for $6 \mathrm{~h}$; step 2: $141 \mathrm{mg}$ phenol (1.50 mmol, 1.00 equiv.), $310 \mathrm{mg}$ DIPEA ( $2.40 \mathrm{mmol}, 1.60$ equiv.); $35{ }^{\circ} \mathrm{C}$ for $46 \mathrm{~h}$; step 3: $261 \mathrm{mg}$ methyl 3-aminobenzoate $(1.73 \mathrm{mmol}, 1.15$ equiv.), $291 \mathrm{mg}$ DIPEA ( $2.25 \mathrm{mmol}, 1.50$ equiv.); $35^{\circ} \mathrm{C}$ for $26 \mathrm{~h}$. After the work-up procedure described in general procedure $\mathrm{B}$, the compound crystallized; the material was then dissolved in $10 \mathrm{~cm}^{3}$ refluxing chloroform and $10 \mathrm{~cm}^{3}$ $n$-hexane was added to the boiling mixture, which caused the compound to precipitate. After crystallization overnight, the compound was isolated by filtration, washed with $30 \mathrm{~cm}^{3} \mathrm{LP}$ and dried in vacuo. Yield: $523 \mathrm{mg}(84 \%)$; appearance: off-white powder; m.p.: $198-199.5^{\circ} \mathrm{C} ; R_{f-}$ $=0.50 \quad$ (hexane $/$ EtOAc, 2:1); ${ }^{1} \mathrm{H}$ NMR $\left(\right.$ DMSO- $d_{6}$, $200 \mathrm{MHz}): \delta=3.81\left(\mathrm{~s}, 3 \mathrm{H}, \mathrm{CH}_{3}\right), 7.18-7.31(\mathrm{~m}, 7 \mathrm{H})$, $7.41-7.48(\mathrm{~m}, 4 \mathrm{H}), 7.56\left(\mathrm{~d}, J_{3}=7.8 \mathrm{~Hz}, 1 \mathrm{H}\right), 7.75(\mathrm{~d}$, $\left.J_{3}=7.9 \mathrm{~Hz}, 1 \mathrm{H}\right), 8.03(\mathrm{~s}, 1 \mathrm{H}, \mathrm{H} 2$ ') $10.42(\mathrm{~s}, 1 \mathrm{H}, \mathrm{NH})$ ppm; ${ }^{13} \mathrm{C}$ NMR (DMSO- $\left.d_{6}, 50 \mathrm{MHz}\right): \delta=52.2\left(\mathrm{q}, \mathrm{CH}_{3}\right.$ ), 121.2 (d, C2'), 121.8 (d, C2" \& C2'” \& C6" \& C6" ), 123.9 (d, C4'), 125.1 (d, C6'), 125.7 (d, C4" \& C4'”), 128.7 (d, C5'), 129.6 (d, C3" \& C3'” \& C5" \& C5'”), 130.0 (s, C3'), 138.9 (s, C1'), 151.8 (s, C1", C1'”), 166.0 (s, C2*), $166.2\left(\mathrm{~s}, \mathrm{CO}_{2}{ }^{*}\right), 171.8 \& 172.2$ (bs, C4 \& C6, rotamers) ppm.

Methyl 3-[[4-(4-chlorophenoxy)-6-phenoxy-1,3,5-triazin-2yl]amino]benzoate $\left(5 \mathrm{c}, \mathrm{C}_{23} \mathrm{H}_{17} \mathrm{CIN}_{4} \mathrm{O}_{4}\right)$ Prepared according to general procedure B using for step 1: $277 \mathrm{mg} \mathrm{2,4,6-}$ trichlorotriazine ( $1.50 \mathrm{mmol}, 1.00$ equiv.), $141 \mathrm{mg}$ phenol (1.50 mmol, 1.00 equiv.), $213 \mathrm{mg}$ DIPEA (1.65 mmol, 1.10 equiv.); $-35^{\circ} \mathrm{C}$ for $6 \mathrm{~h}$; step 2: $193 \mathrm{mg}$ 4-chlorophenol (1.50 mmol, 1.00 equiv.), $310 \mathrm{mg}$ DIPEA (2.40 mmol, 1.60 equiv.); $35^{\circ} \mathrm{C}$ for $46 \mathrm{~h}$; step 3: $261 \mathrm{mg}$ methyl 3-aminobenzoate (1.73 mmol, 1.15 equiv.), $291 \mathrm{mg}$ DIPEA (2.25 mmol, 1.50 equiv.); $35^{\circ} \mathrm{C}$ for $26 \mathrm{~h}$. After the work-up procedure described in general procedure $\mathrm{B}$, approx. $5 \mathrm{~cm}^{3} \mathrm{LP}$ was added to induce precipitation and facilitate evaporation to dryness. The crude material was dissolved in $5.5 \mathrm{~cm}^{3}$ refluxing $\mathrm{CHCl}_{3}$ and $5.5 \mathrm{~cm}^{3} n$-hexane was added. The solution was briefly sonicated to facilitate crystallization and the compound was isolated by filtration, washed with $15 \mathrm{~cm}^{3} \mathrm{LP}$ and dried in vacuo. Yield: $546 \mathrm{mg}$ (81\%); appearance: colorless powder; m.p.:
186.5-188 ${ }^{\circ} \mathrm{C} ; R_{f}=0.48$ (hexane/EtOAc, 2:1); ${ }^{1} \mathrm{H}$ NMR (DMSO- $\left.d_{6}, 400 \mathrm{MHz}\right): \delta=3.82\left(\mathrm{~s}, 3 \mathrm{H}, \mathrm{CH}_{3}\right), 7.19-7.36$ (m, 6H), 7.41-7.53 (m, 4H), $7.58\left(\mathrm{~d}, J_{3}=6 \mathrm{~Hz}, 1 \mathrm{H}\right), 7.76$ (bs, 1H), 8.08 (s, 1H, H2'), 10.47 (s, 1H, NH) ppm; ${ }^{13} \mathrm{C}$ NMR (DMSO- $\left.d_{6}, 100 \mathrm{MHz}, 120{ }^{\circ} \mathrm{C}\right): \delta=51.2\left(\mathrm{q}, \mathrm{CH}_{3}\right)$, 120.9 (d), 121.2 (d), 123.5 (d), 124.8 (d), 127.6 (d), 128.6 (d), 128.7 (d), 129.3 (s), 129.9 (s), 138.1 (s, C1'), 150.3 (s, C1"), 151.6 (s, C1'”), 164.5 (s, C2*), 166.1 (s, $\left.\mathrm{CO}_{2}{ }^{*}\right)$, 171.3 \& 171.5 (s, $\mathrm{C} 4$ \& $\mathrm{C} 6$ ) ppm; one d signal missing possibly due to overlap.

Methyl 3-[[4-(3-chlorophenoxy)-6-phenoxy-1,3,5-triazin-2yl]amino]benzoate (5d, $\left.\mathrm{C}_{23} \mathrm{H}_{17} \mathrm{ClN}_{4} \mathrm{O}_{4}\right)$ Prepared according to general procedure $\mathrm{B}$ using for step 1: $277 \mathrm{mg} 2,4,6$ trichlorotriazine ( $1.50 \mathrm{mmol}, 1.00$ equiv.), $141 \mathrm{mg}$ phenol (1.50 mmol, 1.00 equiv.), $213 \mathrm{mg}$ DIPEA (1.65 mmol, 1.10 equiv.); $-35{ }^{\circ} \mathrm{C}$ for $6 \mathrm{~h}$; step 2: $193 \mathrm{mg}$ 3-chlorophenol (1.50 mmol, 1.00 equiv.), $310 \mathrm{mg}$ DIPEA (2.40 mmol, 1.60 equiv.); $35{ }^{\circ} \mathrm{C}$ for $46 \mathrm{~h}$; step 3: $261 \mathrm{mg}$ methyl 3-aminobenzoate (1.73 mmol, 1.15 equiv.), $291 \mathrm{mg}$ DIPEA (2.25 mmol, 1.50 equiv.); $35{ }^{\circ} \mathrm{C}$ for $26 \mathrm{~h}$. After the work-up procedure described in general procedure B, approx. $5 \mathrm{~cm}^{3} \mathrm{LP}$ was added to induce precipitation and facilitate evaporation to dryness. The crude material was dissolved in $4 \mathrm{~cm}^{3}$ refluxing $\mathrm{CHCl}_{3}$ and $4 \mathrm{~cm}^{3} n$-hexane was added. After cooling and crystallization were complete, the compound was isolated by filtration, washed with $20 \mathrm{~cm}^{3} \mathrm{LP}$, and dried in vacuo. Yield: $600 \mathrm{mg}(89 \%)$; appearance: colorless powder; m.p.: $151.5-156{ }^{\circ} \mathrm{C} ; R_{f-}$ $=0.55$ (hexane $/$ EtOAc, 2:1); ${ }^{1} \mathrm{H}$ NMR (DMSO- $d_{6}$, $200 \mathrm{MHz}): \delta=3.81\left(\mathrm{~s}, 3 \mathrm{H}, \mathrm{CH}_{3}\right), 7.24-7.51(\mathrm{~m}, 10 \mathrm{H})$, $7.58\left(\mathrm{~d}, J_{3}=7.8 \mathrm{~Hz}, 1 \mathrm{H}\right), 7.77\left(\mathrm{~d}, J_{3}=8.3 \mathrm{~Hz}, 1 \mathrm{H}\right), 8.05$ (t, $J=1.6 \mathrm{~Hz}, 1 \mathrm{H}, \mathrm{H} 2$ ') $10.48(\mathrm{~s}, 1 \mathrm{H}, \mathrm{NH}) \mathrm{ppm} ;{ }^{13} \mathrm{C} \mathrm{NMR}$ (DMSO- $\left.d_{6}, 50 \mathrm{MHz}\right): \delta=52.2\left(\mathrm{q}, \mathrm{CH}_{3}\right), 120.7$ (d, C6"), 121.3 (d, C2'), 121.8 (d, C2'” \& C6'”), 122.4 (d, C2"), 124.0 (d, C4'), 125.1 (d, C6'), 125.7 (d, C4'”), 125.9 (d, C4"), 128.7 (d, C5'), 129.6 (d, C3'” \& C5'”), 130.0 (s, C3'), 130.9 (d, C5”), 133.4 (s, C3"), 138.7 (s, C1'), 151.8 (s, C1'”), 152.4 (s, C1"), 166.0 (s, C2*), 166.2 (s, $\left.\mathrm{CO}_{2}{ }^{*}\right)$, 171.3-172.4 (C4 \& C6, not resolved) ppm.

Methyl 3-[[4-(2-chlorophenoxy)-6-phenoxy-1,3,5-triazin-2yl]amino]benzoate $\left(5 \mathrm{e}, \mathrm{C}_{23} \mathrm{H}_{17} \mathrm{ClN}_{4} \mathrm{O}_{4}\right.$ ) Prepared according to general procedure $\mathrm{B}$ using for step 1: $277 \mathrm{mg} \mathrm{2,4,6-}$ trichlorotriazine ( $1.50 \mathrm{mmol}, 1.00$ equiv.), $141 \mathrm{mg}$ phenol (1.50 mmol, 1.00 equiv.), $213 \mathrm{mg}$ DIPEA (1.65 mmol, 1.10 equiv.); $35{ }^{\circ} \mathrm{C}$ for $6 \mathrm{~h}$; step 2: $193 \mathrm{mg}$ 2-chlorophenol (1.50 mmol, 1.00 equiv.), $310 \mathrm{mg}$ DIPEA (2.40 mmol, 1.60 equiv.); $35{ }^{\circ} \mathrm{C}$ for $46 \mathrm{~h}$; step 3: $261 \mathrm{mg}$ methyl 3-aminobenzoate (1.73 mmol, 1.15 equiv.), $291 \mathrm{mg}$ DIPEA (2.25 mmol, 1.50 equiv.); $35{ }^{\circ} \mathrm{C}$ for $26 \mathrm{~h}$. After the work-up procedure described in general procedure $\mathrm{B}$, approx. $5 \mathrm{~cm}^{3} \mathrm{LP}$ was added to induce precipitation and facilitate evaporation to dryness. The crude material was 
dissolved in a refluxing mixture of $\mathrm{CHCl}_{3} / n$-hexane (2: 3 , $\left.11 \mathrm{~cm}^{3}\right)$ and more $n$-hexane $\left(11 \mathrm{~cm}^{3}\right)$ was added. The solution was briefly sonicated to facilitate crystallization and the compound was isolated by filtration, washed with $20 \mathrm{~cm}^{3}$ LP and dried in vacuo to give 5 e $(540 \mathrm{mg}, 80 \%)$. A fraction of this material $(95 \mathrm{mg}$ ) was further purified by column chromatography (MPLC, $100 \mathrm{~g}$ silica, $45 \mathrm{~cm}^{3}$ $\min ^{-1}$ flow rate, LP with a gradient of EtOAc $1-12 \%$ within $15 \mathrm{~min}$, then up to $100 \%$ EtOAc within $60 \mathrm{~min}$ ). Yield: $79 \mathrm{mg}$ (corresponds to $67 \%$ yield over 2 purification steps); appearance: slightly off-white powder; m.p.: 139-141.5 ${ }^{\circ} \mathrm{C} ; R_{f}=0.48$ (hexane/EtOAc, 2:1); ${ }^{1} \mathrm{H}$ NMR $\left(\mathrm{DMSO}-d_{6}, 200 \mathrm{MHz}\right): \delta=3.81\left(\mathrm{~s}, 3 \mathrm{H}, \mathrm{CH}_{3}\right), 7.17-7.48$ $(\mathrm{m}, 9 \mathrm{H}), 7.58\left(\mathrm{t}, J_{3}=8.5 \mathrm{~Hz}, 2 \mathrm{H}\right), 7.69(\mathrm{bs}, 1 \mathrm{H}), 7.99$ (s, $1 \mathrm{H}, \mathrm{H} 2$ ') 10.51 (s, $1 \mathrm{H}, \mathrm{NH}) \mathrm{ppm} ;{ }^{13} \mathrm{C}$ NMR (DMSO- $d_{6}$, $50 \mathrm{MHz}): \delta=52.2$ (q, $\left.\mathrm{CH}_{3}\right), 121.3$ (d, C2'), 121.8 (d, C2', \& C6"”), 124.0 (d, C4'), 124.2 (d, C6"), 125.1 (d, C6'), 125.8 (d, C4"”), 126.0 (s, C2"), 127.5 (d, C4"*), 128.6 (d, C5”*), 128.7 (d, C5'*), 129.6 (d, C3"' \& C5'”), 130.0 (s, C3'), 130.3 (d, C3”), 138.6 (s, C1'), 147.7 (s, C1"), 151.7 (s, C1 $\left.{ }^{\prime \prime}\right), 166.0$ (s, C2*), 166.2 (s, $\left.\mathrm{CO}_{2}{ }^{*}\right), 171.2 \& 171.6$ \& $171.9 \& 172.2$ (s, C4 \& C6, 2 pairs of rotamers) ppm.

Methyl 3-[[4-[4-(1,1-dimethylethyl)phenoxy]-6-phenoxy1,3,5-triazin-2-yl]amino]benzoate (5f, $\left.\mathrm{C}_{27} \mathrm{H}_{26} \mathrm{~N}_{4} \mathrm{O}_{4}\right)$ Prepared according to general procedure $\mathrm{B}$ using for step 1: $277 \mathrm{mg}$ 2,4,6-trichlorotriazine (1.50 mmol, 1.00 equiv.), $141 \mathrm{mg}$ phenol (1.50 mmol, 1.00 equiv.), $213 \mathrm{mg}$ DIPEA (1.65 mmol, 1.10 equiv.); $-35{ }^{\circ} \mathrm{C}$ for $6 \mathrm{~h}$; step 2: $225 \mathrm{mg}$ 4-(1,1-dimethylethyl)phenol (1.50 mmol, 1.00 equiv.), $310 \mathrm{mg}$ DIPEA (2.40 mmol, 1.60 equiv.); $35^{\circ} \mathrm{C}$ for $46 \mathrm{~h}$; step 3: $261 \mathrm{mg}$ methyl 3-aminobenzoate (1.73 mmol, 1.15 equiv.), $291 \mathrm{mg}$ DIPEA (2.25 mmol, 1.50 equiv.); $35^{\circ} \mathrm{C}$ for $26 \mathrm{~h}$. After the work-up procedure described in general procedure $\mathrm{B}$, the solvent was evaporated completely and the crude material was dissolved in a refluxing mixture of $\mathrm{CHCl}_{3} / n$-hexane $\left(1: 1,4.5 \mathrm{~cm}^{3}\right)$ and more $n$-hexane $\left(8.5 \mathrm{~cm}^{3}\right)$ was added. The solution was briefly sonicated to facilitate crystallization and the compound was isolated by filtration, washed with $60 \mathrm{~cm}^{3} \mathrm{LP}$, and dried in vacuo to give $5 \mathbf{f}(565 \mathrm{mg}, 80 \%)$. A fraction of this material (235 mg) was further purified by column chromatography (MPLC, $100 \mathrm{~g}$ silica, $45 \mathrm{~cm}^{3} \mathrm{~min}^{-1}$ flow rate, LP with a gradient of EtOAc 1 to $12 \%$ within $15 \mathrm{~min}$, then up to 100\% EtOAc within $60 \mathrm{~min}$ ). Yield: $194 \mathrm{mg}$ (corresponds to $66 \%$ yield over 2 purification steps); appearance: colorless solid; m.p.: $85-86{ }^{\circ} \mathrm{C} ; R_{f}=0.53$ (hexane/EtOAc, 2:1); ${ }^{1} \mathrm{H}$ NMR (DMSO- $\left.d_{6}, 200 \mathrm{MHz}\right): \delta=1.28$ (s, 9H, $\left.\mathrm{C}\left(\mathrm{CH}_{3}\right)_{3}\right), 3.82\left(\mathrm{~s}, 3 \mathrm{H}, \mathrm{OCH}_{3}\right), 7.14-7.30(\mathrm{~m}, 6 \mathrm{H})$, $7.41-7.48(\mathrm{~m}, 4 \mathrm{H}), 7.56\left(\mathrm{~d}, J_{3}=7.7 \mathrm{~Hz}, 1 \mathrm{H}\right), 7.76(\mathrm{~d}$, $\left.J_{3}=7.9 \mathrm{~Hz}, 1 \mathrm{H}\right), 8.04\left(\mathrm{~s}, 1 \mathrm{H}, \mathrm{H} 2^{\prime}\right), 10.41(\mathrm{~s}, 1 \mathrm{H}, \mathrm{NH})$ ppm; ${ }^{13} \mathrm{C}$ NMR (DMSO- $\left.d_{6}, 50 \mathrm{MHz}\right): \delta=31.2 \quad$ (q, $\left.\mathrm{C}\left(\mathrm{CH}_{3}\right)_{3}\right), 34.2\left(\mathrm{~s}, \mathrm{C}\left(\mathrm{CH}_{3}\right)_{3}\right), 52.2\left(\mathrm{OCH}_{3}\right), 121.2$ (d, $\mathrm{C} 2$ ”
\& C6"), 121.3 (d, C2'), 121.8 (d, C2'” \& C6" "), 123.9 (d, C4'), 125.1 (d, C6'), 125.7 (d, C4'”), 126.3 (d, C3" \& C5”), 128.7 (d, C5'), 129.6 (d, C3"” \& C5"”), 130.0 (s, C3'), 138.9 (s, C1'), 148.0 (s, C4”*), 149.5 (s, C1"*), 151.8 (s, C1'”), 166.0 (s, C2*), $166.2\left(\mathrm{~s}, \mathrm{CO}_{2}^{*}\right)$, 171.7-172.5 (C4 \& C6, not resolved) ppm.

Methyl 3-[[4-(4-methoxyphenoxy)-6-phenoxy-1,3,5-triazin2-yl]amino]benzoate $\left(5 \mathrm{~g}, \mathrm{C}_{24} \mathrm{H}_{20} \mathrm{~N}_{4} \mathrm{O}_{5}\right)$ Prepared according to general procedure $\mathrm{B}$ using for step 1: $277 \mathrm{mg} \mathrm{2,4,6-}$ trichlorotriazine (1.50 mmol, 1.00 equiv.), $141 \mathrm{mg}$ phenol (1.50 mmol, 1.00 equiv.), $213 \mathrm{mg}$ DIPEA (1.65 mmol, 1.10 equiv.); $\quad-35^{\circ} \mathrm{C}$ for $7 \mathrm{~h}$; step 2: $186 \mathrm{mg}$ 4-methoxyphenol (1.50 mmol, 1.00 equiv.), $310 \mathrm{mg}$ DIPEA (2.40 mmol, 1.60 equiv.); $35^{\circ} \mathrm{C}$ for $47 \mathrm{~h}$; step 3: $261 \mathrm{mg}$ methyl 3 -aminobenzoate (1.73 mmol, 1.15 equiv.), $291 \mathrm{mg}$ DIPEA (2.25 mmol, 1.50 equiv.); $35^{\circ} \mathrm{C}$ for $23.5 \mathrm{~h}$. After the work-up procedure described in general procedure $\mathrm{B}$, the compound was purified by manual column chromatography (silica, EtOAc/LP) and dried in vacuo. Yield: $569 \mathrm{mg}$ (85\%); appearance: colorless solid; m.p.: 56-60 ${ }^{\circ} \mathrm{C} ; R_{f}=0.36$ (hexane/EtOAc, $2: 1$ ); ${ }^{1} \mathrm{H}$ NMR $\left(\mathrm{DMSO}-d_{6}, 200 \mathrm{MHz}\right): \delta=3.76\left(\mathrm{~s}, 3 \mathrm{H}, \mathrm{C} 4{ }^{\prime} \mathrm{OCH}_{3}\right), 3.81$ (s, 3H, $\left.\mathrm{CO}_{2} \mathrm{CH}_{3}\right), 6.96(\mathrm{~d}, J=9.0 \mathrm{~Hz}, 2 \mathrm{H}, \mathrm{H} 3 "$ \& $\mathrm{H} 5 ")$, 7.13-7.31 (m, 6H), 7.41-7.48 (m, 2H), $7.56 \quad(\mathrm{~d}$, $\left.J_{3}=7.9 \mathrm{~Hz}, 1 \mathrm{H}\right), 7.76(\mathrm{~d}, J=7.9 \mathrm{~Hz}, 1 \mathrm{H}), 8.04(\mathrm{~s}, 1 \mathrm{H}$, $\mathrm{H} 2$ '), 10.39 (s, 1H, NH) ppm; ${ }^{13} \mathrm{C}$ NMR (DMSO- $d_{6}$, $50 \mathrm{MHz}): \delta=52.1$ (q, $\left.\mathrm{CO}_{2} \mathrm{CH}_{3}\right), 55.4$ (q, C4" $\left.{ }^{\prime} \mathrm{OCH}_{3}\right)$, 114.4 (d, C3" \& C5"), 121.1 (d, C2'), 121.7 (d, C2'” \& C6"”), 122.6 (d, C2" \& C6"), 123.8 (d, C4'), 125.0 (d, C6'), 125.6 (d, C4'”), 128.6 (d, C5'), 129.5 (d, C3'” \& C5'”), 129.9 (s, C3'), 138.8 (s, C1'), 145.2 (s, C1"), 151.8 (s, C1'”), 156.8 (s, C4”), 165.9 (s, C2*), 166.1 (s, $\left.\mathrm{CO}_{2}^{*}\right)$, 171.7-172.5 (C4 \& C6, not resolved) ppm.

Methyl 3-[[4-(4-cyanophenoxy)-6-phenoxy-1,3,5-triazin-2yl]amino]benzoate $\left(5 \mathrm{~h}, \mathrm{C}_{24} \mathrm{H}_{17} \mathrm{~N}_{5} \mathrm{O}_{4}\right)$ Prepared according to general procedure B using for step 1: $277 \mathrm{mg}$ 2,4,6trichlorotriazine (1.50 mmol, 1.00 equiv.), $141 \mathrm{mg}$ phenol (1.50 mmol, 1.00 equiv.), $213 \mathrm{mg}$ DIPEA (1.65 mmol, 1.10 equiv.); $-35^{\circ} \mathrm{C}$ for $6 \mathrm{~h}$; step 2: $179 \mathrm{mg}$ 4-hydroxybenzonitrile (1.50 mmol, 1.00 equiv.), $310 \mathrm{mg}$ DIPEA (2.40 mmol, 1.60 equiv.); $35^{\circ} \mathrm{C}$ for $46 \mathrm{~h}$; step 3: $261 \mathrm{mg}$ methyl 3-aminobenzoate (1.73 mmol, 1.15 equiv.), $291 \mathrm{mg}$ DIPEA (2.25 mmol, 1.50 equiv.); $35^{\circ} \mathrm{C}$ for $26 \mathrm{~h}$. After the work-up procedure described in general procedure $\mathrm{B}$, approx. $10 \mathrm{~cm}^{3}$ LP was added to induce precipitation (under sonication) and facilitate evaporation to dryness. The crude material was dissolved in $16 \mathrm{~cm}^{3}$ refluxing $\mathrm{CHCl}_{3}$ and $16 \mathrm{~cm}^{3} n$-hexane was added. After cooling and crystallization were complete, the compound was isolated by filtration, washed with $20 \mathrm{~cm}^{3} \mathrm{LP}$, and dried in vacuo to give $\mathbf{5 h}$ (569 mg, 86\%). A fraction of this material (223 mg) was further purified by column chromatography 
(MPLC, $100 \mathrm{~g}$ silica, $45 \mathrm{~cm}^{3} \mathrm{~min}^{-1}$ flow rate, LP with a gradient of EtOAc 1 to $12 \%$ within $17 \mathrm{~min}$, then up to 100\% EtOAc within $60 \mathrm{~min}$ ). Yield: $183 \mathrm{mg}$ (corresponds to $71 \%$ yield over 2 purification steps); appearance: colorless solid; m.p.: $116.5-120{ }^{\circ} \mathrm{C} ; R_{f}=0.40$ (hexane/ EtOAc, $2: 1$ ); ${ }^{1} \mathrm{H}$ NMR (DMSO- $d_{6}, 200 \mathrm{MHz}$ ): $\delta=3.82(\mathrm{~s}$, $\left.3 \mathrm{H}, \mathrm{CH}_{3}\right), 7.24-7.31(\mathrm{~m}, 4 \mathrm{H}), 7.41-7.60(\mathrm{~m}, 5 \mathrm{H}), 7.71$ (bs, $1 \mathrm{H}), 7.94\left(\mathrm{~d}, J_{3}=8.1 \mathrm{~Hz}, 2 \mathrm{H}, \mathrm{H} 3\right.$ " \& H5"), 8.03 (s, $1 \mathrm{H}$, $\left.\mathrm{H} 2^{\prime}\right), 10.50(\mathrm{~s}, 1 \mathrm{H}, \mathrm{NH}) \mathrm{ppm} ;{ }^{13} \mathrm{C}$ NMR (DMSO- $d_{6}$, $50 \mathrm{MHz}): \delta=52.1$ (q, $\left.\mathrm{CH}_{3}\right), 108.5$ (s, C4"), $118.4(\mathrm{~s}, \mathrm{CN})$, 121.2 (d, C2'), 121.7 (d, C2'” \& C6'”), 123.2 (d, C2" \& C6”), 124.0 (d, C4'), 125.1 (d, C6'), 125.7 (d, C4'”), 128.6 (d, C5'), 129.5 (d, C3'” \& C5'”), 129.9 (s, C3'), 134.0 (d, C3" \& C5"), 138.5 (s, C1'), 151.7 (s, C1"”), 155.1 (s, C1"), 165.8 (s, C2*), 166.0 (s, $\mathrm{CO}_{2} *$ ), 170.9-172.3 (C4 \& $\mathrm{C} 6$, not resolved) $\mathrm{ppm}$.

\section{Methyl 3-[[4-(3-nitrophenoxy)-6-phenoxy-1,3,5-triazin-2-} yl]amino]benzoate ( $5 \mathrm{i}, \mathrm{C}_{23} \mathrm{H}_{17} \mathrm{~N}_{5} \mathrm{O}_{6}$ ) Prepared according to general procedure B using for step 1: $277 \mathrm{mg} \mathrm{2,4,6-}$ trichlorotriazine ( $1.50 \mathrm{mmol}, 1.00$ equiv.), $141 \mathrm{mg}$ phenol (1.50 mmol, 1.00 equiv.), $213 \mathrm{mg}$ DIPEA (1.65 mmol, 1.10 equiv.); $-35^{\circ} \mathrm{C}$ for $6 \mathrm{~h}$; step 2: $209 \mathrm{mg}$ 3-nitrophenol (1.50 mmol, 1.00 equiv.), $310 \mathrm{mg}$ DIPEA ( $2.40 \mathrm{mmol}$, 1.60 equiv.); $40{ }^{\circ} \mathrm{C}$ for $46 \mathrm{~h}$; step 3: $261 \mathrm{mg}$ methyl 3-aminobenzoate (1.73 mmol, 1.15 equiv.), $291 \mathrm{mg}$ DIPEA ( $2.25 \mathrm{mmol}, 1.50$ equiv.); $40{ }^{\circ} \mathrm{C}$ for $24 \mathrm{~h}$. After the work-up procedure described in general procedure $\mathrm{B}$, approx. $5 \mathrm{~cm}^{3}$ diethyl ether was added to induce precipitation and facilitate evaporation to dryness. The crude material was dissolved in $8 \mathrm{~cm}^{3}$ refluxing $\mathrm{CHCl}_{3}$ and $8 \mathrm{~cm}^{3} n$-hexane was added. The solution was briefly sonicated to facilitate crystallization and the compound was isolated by filtration and washed with $60 \mathrm{~cm}^{3} \mathrm{LP}$. The compound had to be re-crystallized a second time by dissolving it in $17 \mathrm{~cm}^{3}$ refluxing $\mathrm{CHCl}_{3}$ and adding $17 \mathrm{~cm}^{3} n$ hexane. After complete crystallization, the material was isolated by filtration, washed with $25 \mathrm{~cm}^{3} \mathrm{LP}$ and dried in vacuo. Yield: $474 \mathrm{mg}$ (69\%); appearance: colorless powder; m.p.: $165-176{ }^{\circ} \mathrm{C} ; R_{f}=0.34$ (hexane/EtOAc, $\left.2: 1\right) ;{ }^{1} \mathrm{H}$ NMR (DMSO- $\left.d_{6}, 200 \mathrm{MHz}\right): \delta=3.80\left(\mathrm{~s}, 3 \mathrm{H}, \mathrm{CH}_{3}\right)$, 7.21-7.29 (m, 4H), 7.39-7.46 (m, 2H), $7.57 \quad(\mathrm{~d}$, $\left.J_{3}=7.5 \mathrm{~Hz}, 1 \mathrm{H}\right), 7.68-7.80(\mathrm{~m}, 3 \mathrm{H}), 8.05(\mathrm{~s}, 1 \mathrm{H}, \mathrm{H} 2$ '), $8.14\left(\mathrm{~d}, J_{3}=7.3 \mathrm{~Hz}, 1 \mathrm{H}\right), 8.21$ (s, 1H, H2"), 10.51 (s, $1 \mathrm{H}$, $\mathrm{NH}) \mathrm{ppm} ;{ }^{13} \mathrm{C}$ NMR (DMSO- $\left.d_{6}, 50 \mathrm{MHz}\right): \delta=52.1(\mathrm{q}$, $\mathrm{CH}_{3}$ ), 117.4 (d, C2"), 120.6 (d, C4”), 121.2 (d, C2'), 121.7 (d, C2'” \& C6'”), 123.9 (d, C4'), 125.1 (d, C6'), 125.7 (d, C4" "), 128.6 (d, C6"*), 128.7 (d, C5'*), 129.4 (d, C3'” \& C5'”), 129.9 (s, C3'), 130.7 (d, C5”), 138.5 (s, C1'), 148.3 (s, C3"), 151.7 (s, C1"”*), 151.9 (s, C1"*), 165.8 (s, C2*), $166.1\left(\mathrm{~s}, \mathrm{CO}_{2}{ }^{*}\right), 171.1-172.3$ (C4 \& $\mathrm{C} 6$, not resolved) ppm.
Methyl 3-[[4-(4-formyl-2-methoxyphenoxy)-6-phenoxy1,3,5-triazin-2-yl]amino]benzoate $\left(5 \mathrm{j}, \mathrm{C}_{25} \mathrm{H}_{20} \mathrm{~N}_{4} \mathrm{O}_{6}\right)$ Prepared according to general procedure $\mathrm{B}$ using for step 1 : $277 \mathrm{mg}$ 2,4,6-trichlorotriazine ( $1.50 \mathrm{mmol}, 1.00$ equiv.), $141 \mathrm{mg}$ phenol (1.50 mmol, 1.00 equiv.), $213 \mathrm{mg}$ DIPEA (1.65 mmol, 1.10 equiv.); $-35^{\circ} \mathrm{C}$ for $6 \mathrm{~h}$; step $2: 228 \mathrm{mg}$ vanillin (1.50 mmol, 1.00 equiv.), $310 \mathrm{mg}$ DIPEA (2.40 mmol, 1.60 equiv.); $40{ }^{\circ} \mathrm{C}$ for $46 \mathrm{~h}$; step 3: $261 \mathrm{mg}$ methyl 3-aminobenzoate (1.73 mmol, 1.15 equiv.), $291 \mathrm{mg}$ DIPEA (2.25 mmol, 1.50 equiv.); $40{ }^{\circ} \mathrm{C}$ for $24 \mathrm{~h}$. After the work-up procedure described in general procedure $\mathrm{B}$, $2 \mathrm{~cm}^{3}$ diethyl ether was added, followed by approx $5 \mathrm{~cm}^{3}$ LP to induce crystallization and facilitate evaporation to dryness. As re-crystallization from chloroform $/ n$-hexane was not successful, the compound was purified via column chromatography (MPLC, $90 \mathrm{~g}$ silica, $45 \mathrm{~cm}^{3} \mathrm{~min}^{-1}$ flow rate, $\mathrm{CH}_{2} \mathrm{Cl}_{2}$ with a gradient of EtOAc (1 to $4 \%$ within $60 \mathrm{~min})$ and dried in vacuo. Yield: $545 \mathrm{mg}(77 \%)$; appearance: colorless crystals; m.p.: $79-82{ }^{\circ} \mathrm{C} ; R_{f}=0.24$ (hexane/EtOAc, 2:1); ${ }^{1} \mathrm{H}$ NMR (DMSO- $d_{6}, 200 \mathrm{MHz}$ ): $\delta=3.80\left(\mathrm{~s}, 3 \mathrm{H}, \mathrm{CO}_{2} \mathrm{CH}_{3}\right), 3.85\left(\mathrm{~s}, 3 \mathrm{H}, \mathrm{C}^{\prime}{ }^{\prime} \mathrm{OCH}_{3}\right)$, 7.17-7.30 (m, 4H), 7.40-7.70 (m, 7H), 7.99 (s, 1H, H2'), $10.00(\mathrm{~s}, 1 \mathrm{H}, \mathrm{CHO}), 10.46(\mathrm{~s}, 1 \mathrm{H}, \mathrm{NH}) \mathrm{ppm} ;{ }^{13} \mathrm{C} \mathrm{NMR}$ (DMSO- $\left.d_{6}, 50 \mathrm{MHz}\right): \delta=52.1\left(\mathrm{q}, \mathrm{CO}_{2} \mathrm{CH}_{3}\right), 56.0(\mathrm{q}$, C2" $\mathrm{OCH}_{3}$ ), 112.1 (d, C3"), 121.2 (d, C2'), 121.7 (d, C2'” \& C6"”), $123.5\left(\mathrm{~d}, \mathrm{C} 5{ }^{*}\right), 123.7\left(\mathrm{~d}, \mathrm{C} 4{ }^{*}\right), 123.9$ (d, C6"*), 125.0 (d, C6'), 125.7 (d, C4'”), 128.6 (d, C5'), 129.5 (d, C3'” \& C5'”), 129.9 (s, C3'), 135.0 (s, C4"), 138.6 (s, C1'), 145.2 (s, C1"*), 151.68 (s, C2"*), 151.76 (s, C1'”*), 165.8 (s, C2*), $166.0\left(\mathrm{~s}, \mathrm{CO}_{2} *\right), 171.2-172.4$ (C4 \& C6, not resolved), 191.9 (d, CHO) ppm.

\section{Methyl 3-[[4-(4-formyl-2-methoxyphenoxy)-6-(4-} methoxyphenoxy)-1,3,5-triazin-2-yl]amino]benzoate (5k, $\mathrm{C}_{26} \mathrm{H}_{22} \mathrm{~N}_{4} \mathrm{O}_{7}$ ) Prepared according to general procedure $\mathrm{B}$ using for step 1: $277 \mathrm{mg}$ 2,4,6-trichlorotriazine (1.50 mmol, 1.00 equiv.), $186 \mathrm{mg}$ 4-methoxyphenol (1.50 mmol, 1.00 equiv.), $213 \mathrm{mg}$ DIPEA (1.65 mmol, 1.10 equiv.); $-35{ }^{\circ} \mathrm{C}$ for $7 \mathrm{~h}$; step 2: $228 \mathrm{mg}$ vanillin (1.50 mmol, 1.00 equiv.), $310 \mathrm{mg}$ DIPEA (2.40 mmol, 1.60 equiv.); $40{ }^{\circ} \mathrm{C}$ for $47 \mathrm{~h}$; step 3: $261 \mathrm{mg}$ methyl 3-aminobenzoate (1.73 mmol, 1.15 equiv.), $291 \mathrm{mg}$ DIPEA (2.25 mmol, 1.50 equiv.); $40{ }^{\circ} \mathrm{C}$ for $23.5 \mathrm{~h}$. After the work-up procedure described in general procedure B, the was compound was purified via column chromatography (MPLC, $90 \mathrm{~g}$ silica, $30-35 \mathrm{~cm}^{3} \mathrm{~min}^{-1}$ flow rate, $\mathrm{CH}_{2} \mathrm{Cl}_{2}$ with a gradient of EtOAc 1 to $20 \%$ ). Mixed fractions were also obtained, which were re-submitted to another chromatographic purification (MPLC, $40 \mathrm{~g}$ silica, $35 \mathrm{~cm}^{3} \mathrm{~min}^{-1}$ flow rate, $\mathrm{CH}_{2} \mathrm{Cl}_{2}$ with $4 \%$ EtOAc) and dried in vacuo. Yield: $674 \mathrm{mg}(90 \%)$; appearance: colorless solid; m.p.: $79-82{ }^{\circ} \mathrm{C} ; R_{f}=0.18$ (hexane/EtOAc, 2:1); ${ }^{1} \mathrm{H} \quad \mathrm{NMR} \quad\left(\mathrm{DMSO}-d_{6}, \quad 200 \mathrm{MHz}\right): \delta=3.76 \quad(\mathrm{~s}, \quad 3 \mathrm{H}$, 
$\mathrm{C} 4$ " "OCH 3$), 3.80$ (s, 3H, $\left.\mathrm{CO}_{2} \mathrm{CH}_{3} *\right), 3.84$ (s, 3H, $\left.\mathrm{C}^{2}{ }^{\prime} \mathrm{OCH}_{3}{ }^{*}\right), 6.95\left(\mathrm{~d}, J_{3}=9.0 \mathrm{~Hz}, 2 \mathrm{H}, \mathrm{H} 3{ }^{\prime \prime}\right.$ \& $\left.\mathrm{H}^{\prime}{ }^{\prime \prime}\right)$, 7.13-7.26 (m, 3H), 7.45-7.74 (m, 5H), 8.00 (s, 1H, H2'), 10.00 (s, 1H, CHO), $10.44(\mathrm{~s}, 1 \mathrm{H}, \mathrm{NH}) \mathrm{ppm} ;{ }^{13} \mathrm{C} \mathrm{NMR}$ $\left(\mathrm{DMSO}-d_{6}, 50 \mathrm{MHz}\right): \delta=52.1\left(\mathrm{q}, \mathrm{CO}_{2} \mathrm{CH}_{3}\right), 55.4 \quad(\mathrm{q}$, C4" "OCH $\left.{ }_{3}\right), 56.1$ (q, C2" $\left.\mathrm{OCH}_{3}\right), 112.2$ (d, C3"), 114.4 (d, C3" " \& C5" "), 121.3 (d, C2'), 122.6 (d, C2'" \& C6"'), 123.6 (d, C5”*), 123.8 (d, C4”*), 124.0 (d, C6”*), 125.1 (d, C6'), 128.7 (d, C5'), 130.0 (s, C3'), 135.0 (s, C4”), 138.7 (s, C1'), 145.2 (s, C1"*), 145.3 (s, C1 " **), 151.7 (s, C2”), 156.9 (s, C4" "), 166.0 (s, C2*), 166.2 (s, $\left.\mathrm{CO}_{2}{ }^{*}\right), 171.5$ \& 172.3 (bs, C4 \& C6), 192.0 (d, CHO) ppm.

Methyl 3-[[4-(4-bromo-2-formylphenoxy)-6-(4-methoxyphenoxy)-1,3,5-triazin-2-yl]amino]benzoate (5I, $\mathrm{C}_{25} \mathrm{H}_{19} \mathrm{BrN}_{4} \mathrm{O}_{6}$ ) Prepared according to general procedure B using for step 1: $277 \mathrm{mg}$ 2,4,6-trichlorotriazine (1.50 mmol, 1.00 equiv.), $186 \mathrm{mg}$ 4-methoxyphenol (1.50 mmol, 1.00 equiv.), $213 \mathrm{mg}$ DIPEA (1.65 mmol, 1.10 equiv.); $-35^{\circ} \mathrm{C}$ for $6 \mathrm{~h}$; step 2: $302 \mathrm{mg}$ 5-bromo-2-hydroxybenzaldehyde (1.50 mmol, 1.00 equiv.), $310 \mathrm{mg}$ DIPEA (2.40 mmol, 1.60 equiv.); $40{ }^{\circ} \mathrm{C}$ for $46 \mathrm{~h}$; step 3: $261 \mathrm{mg}$ methyl 3-aminobenzoate (1.73 mmol, 1.15 equiv.), $291 \mathrm{mg}$ DIPEA (2.25 mmol, 1.50 equiv.); $40{ }^{\circ} \mathrm{C}$ for $24 \mathrm{~h}$. After the work-up procedure described in general procedure $\mathrm{B}$, the compound was purified via column chromatography (MPLC, $90 \mathrm{~g}$ silica, $35 \mathrm{~cm}^{3} \mathrm{~min}^{-1}$ flow rate, $\mathrm{CH}_{2} \mathrm{Cl}_{2}$ with $1 \%$ EtOAc, gradually increasing to $3 \%$ over the course of the separation), which gave $263 \mathrm{mg}$ (32\%) of still significantly contaminated material. Thus, $46 \mathrm{mg}$ of the material were submitted to another chromatographic purification (MPLC, $105 \mathrm{~g}$ silica, $50 \mathrm{~cm}^{3} \mathrm{~min}^{-1}$ flow rate, LP/EtOAc, 4:1) and then dried in vacuo. Yield: $22 \mathrm{mg}$ (corresponds to $15 \%$ yield over 2 purification steps); appearance: colorless solid; m.p.: $180{ }^{\circ} \mathrm{C}$ (decomp.); $R_{f}=0.31$ (hexane/EtOAc, 2:1); ${ }^{1} \mathrm{H}$ NMR (DMSO- $\left.d_{6}, 200 \mathrm{MHz}\right): \delta=3.76(\mathrm{~s}, 3 \mathrm{H}$, $\left.\mathrm{OCH}_{3}\right), 3.82\left(\mathrm{~s}, 3 \mathrm{H}, \mathrm{OCH}_{3}\right), 6.96\left(\mathrm{~d}, J_{3}=8.9 \mathrm{~Hz}, 2 \mathrm{H}\right)$, $7.10-7.34(\mathrm{~m}, 3 \mathrm{H}), 7.43\left(\mathrm{~d}, J_{3}=8.5 \mathrm{~Hz}, 1 \mathrm{H}\right), 7.53-7.81$ (m, 2H), 7.89-8.10 (m, 3H), 10.00 (s, 1H, CHO), 10.45 (s, $1 \mathrm{H}, \mathrm{NH}) \mathrm{ppm} ;{ }^{13} \mathrm{C}$ NMR (DMSO- $d_{6}, 50 \mathrm{MHz}$ ): $\delta=52.2$ (q, $\left.\mathrm{CO}_{2} \mathrm{CH}_{3}\right), 55.4$ (q, C4' ' $\left.\mathrm{OCH}_{3}\right), 114.4$ (d, C3', \& C5'”), 118.9 (s, C4"), 121.3 (d, C2'), 122.6 (d, C2'” \& C6"”), 124.1 (d, C4'), 125.1 (d, C6'), 126.2 (d, C6”*), 128.7 (d, C5'), 129.8 (s, C2”), 130.0 (s, C3'), 132.5 (d, C3"*), 138.2 (d, C5"*), 138.6 (s, C1'), 145.2 (s, C1"”), 151.7 (s, C1"), 156.9 (s, C4"”), 165.9 (s, C2*), 166.0 (s, $\mathrm{CO}_{2}{ }^{*}$ ), 171.5-172.5 (C4 \& C6, not resolved), 188.4 (d, $\mathrm{CHO}) \mathrm{ppm}$.

Methyl 3-[[4-(4-cyanophenoxy)-6-(4-methoxyphenoxy)1,3,5-triazin-2-yl]amino]benzoate $\left(5 \mathrm{~m}, \mathrm{C}_{25} \mathrm{H}_{19} \mathrm{~N}_{5} \mathrm{O}_{5}\right)$ Prepared according to general procedure $\mathrm{B}$ using for step 1: $277 \mathrm{mg}$ 2,4,6-trichlorotriazine (1.50 mmol, 1.00 equiv.), $186 \mathrm{mg}$ 4-methoxyphenol (1.50 mmol, 1.00 equiv.),
$213 \mathrm{mg}$ DIPEA (1.65 mmol, 1.10 equiv.); $-35{ }^{\circ} \mathrm{C}$ for $7 \mathrm{~h}$; step 2: $179 \mathrm{mg}$ 4-hydroxybenzonitrile (1.50 mmol, 1.00 equiv.), $310 \mathrm{mg}$ DIPEA (2.40 mmol, 1.60 equiv.); $40{ }^{\circ} \mathrm{C}$ for $47 \mathrm{~h}$; step 3: $261 \mathrm{mg}$ methyl 3-aminobenzoate (1.73 mmol, 1.15 equiv.), $291 \mathrm{mg}$ DIPEA (2.25 mmol, 1.50 equiv.); $40{ }^{\circ} \mathrm{C}$ for $23.5 \mathrm{~h}$. After the work-up procedure described in general procedure $\mathrm{B}$, the solvent was evaporated completely and the crude material was dissolved in a refluxing mixture of $\mathrm{CHCl}_{3} /$ cyclohexane $\left(4: 1,7.5 \mathrm{~cm}^{3}\right)$ and more cyclohexane $\left(18 \mathrm{~cm}^{3}\right)$ was added. After cooling and crystallization were completed, the compound was isolated by filtration, washed with $25 \mathrm{~cm}^{3} \mathrm{LP}$, and dried in vacuo. The material had to be purified further by column chromatography (MPLC, silica, short column, neat $\mathrm{CH}_{2} \mathrm{Cl}_{2}$, $20 \mathrm{~cm}^{3} \min ^{-1}$ flow rate, switching to $30 \mathrm{~cm}^{3} \mathrm{~min}^{-1}$ after $1 \mathrm{~h}$, adding $2 \%$ EtOAC after $1 \mathrm{~h} 20 \mathrm{~min}$ and switching to $40 \mathrm{~cm}^{3}$ min after the appearance of the product peak $(1 \mathrm{~h}$ $35 \mathrm{~min})$ ). The solvent was evaporated; TLC analysis revealed a byproduct still present. Thus, the compound was submitted to a second short column (MPLC, silica, $25 \mathrm{~cm}^{3}$ $\min ^{-1}$ flow rate, $\mathrm{CH}_{2} \mathrm{Cl}_{2}$ with $2 \%$ EtOAc, increasing gradually to $10 \%$ with the appearance of the product peak), and dried in vacuo. Yield: $617 \mathrm{mg}(88 \%)$; appearance: colorless powder; m.p.: $132-134.5{ }^{\circ} \mathrm{C} ; R_{f}=0.29$ (hexane/ EtOAc, 2:1); ${ }^{1} \mathrm{H}$ NMR (DMSO- $\left.d_{6}, 200 \mathrm{MHz}\right): \delta=3.76(\mathrm{~s}$, $3 \mathrm{H}, \mathrm{C} 4$ " " $\left.\mathrm{OCH}_{3}\right), 3.82\left(\mathrm{~s}, 3 \mathrm{H}, \mathrm{CO}_{2} \mathrm{CH}_{3}\right), 6.96(\mathrm{~d}$, $J_{3}=9.0 \mathrm{~Hz}, 2 \mathrm{H}, \mathrm{H} 3$ ', \& H5" "), 7.17 (d, $\left.J_{3}=9 \mathrm{~Hz}, 2 \mathrm{H}\right)$, $7.28\left(\mathrm{t}, J_{3}=7.9 \mathrm{~Hz}, 1 \mathrm{H}\right), 7.50\left(\mathrm{~d}, J_{3}=8.6 \mathrm{~Hz}, 2 \mathrm{H}\right), 7.58$ $\left(\mathrm{d}, J_{3}=7.8 \mathrm{~Hz}, 1 \mathrm{H}\right), 7.63-7.82(\mathrm{~m}, 1 \mathrm{H}), 7.94 \quad(\mathrm{~d}$, $J_{3}=8.3 \mathrm{~Hz}, 2 \mathrm{H}, \mathrm{H} 3$ ” \& H5”), 8.05 (s, 1H, H2'), 10.47 (s, $1 \mathrm{H}, \mathrm{NH}) \mathrm{ppm} ;{ }^{13} \mathrm{C} \mathrm{NMR}\left(\mathrm{DMSO}-d_{6}, 50 \mathrm{MHz}\right): \delta=52.1$ (q, $\mathrm{CO}_{2} \mathrm{CH}_{3}$ ), 55.4 (q, C4'" $\mathrm{OCH}_{3}$ ), 108.6 (s, C4"), 114.5 (d, C3'” \& C5'”), 118.5 (s, CN), 121.3 (d, C2'), 122.6 (d, C2'" \& C6"”), 123.2 (d, C2" \& C6"), 124.1 (d, C4'), 125.2 (d, C6'), 128.7 (d, C5'), 130.0 (s, C3'), 134.1 (d, C3” \& C5"), 138.7 (s, C1'), 145.2 (s, C1'”), 155.2 (s, C1"), 156.9 (s, C4" "), 165.9 (s, C2*), $166.1\left(\mathrm{~s}, \mathrm{CO}_{2} *\right), 171.2 \& 171.6$ \& $172.1 \& 172.5$ (s, C4 \& C6, 2 pairs of rotamers) ppm.

\section{Synthesis of 2,4,6-trisubstitued 1,3,5-triazines- general procedure $C$}

The reaction was performed in an $8 \mathrm{~cm}^{3}$ glass vial, using a cryo or thermo block. DIPEA (523 mg, $4.05 \mathrm{mmol}, 2.70$ equiv.) was added to $282 \mathrm{mg}$ phenol (3.00 mmol, 2.00 equiv.), dissolved in $1.0 \mathrm{~cm}^{3} \mathrm{THF}$, and this solution was then added dropwise to a solution of $277 \mathrm{mg} 2,4,6$ trichlorotriazine (1.50 mmol, 1.00 equiv.) in $2.5 \mathrm{~cm}^{3} \mathrm{THF}$ at $-35^{\circ} \mathrm{C}$ while stirring. To ensure complete transfer, another $0.5 \mathrm{~cm}^{3}$ of THF was used to flush all phenol into the reaction mixture. Stirring was continued at $-35{ }^{\circ} \mathrm{C}$ for $2 \mathrm{~h}$, then at r. t. for $70 \mathrm{~h}$ (step 1) until TLC indicated complete conversion or no significant change in reaction 
composition. Then, $291 \mathrm{mg}$ DIPEA (2.25 mmol, 1.50 equiv.) was added directly to the reaction mixture, followed by nucleophile PhXH (1.73 mmol, 1.15 equiv.). The reaction was continued at $\mathrm{r}$. t. for $24 \mathrm{~h}$ (step 2) and checked by TLC. For work-up, $15 \mathrm{~cm}^{3} \mathrm{CH}_{2} \mathrm{Cl}_{2}$ was added to the reaction mixture, followed by $15 \mathrm{~cm}^{3} 1 \mathrm{~N} \mathrm{HCl}_{\mathrm{aq}}$ and the compound was extracted. The layers were separated, the organic phase washed with water $\left(2 \times 15 \mathrm{~cm}^{3}\right)$ and then concentrated in vacuo. Further work-up and purification procedures are given at the respective examples.

4,6-Diphenoxy-N-phenyl-1,3,5-triazin-2-amine $\left(6, \mathrm{C}_{21} \mathrm{H}_{16} \mathrm{~N}_{4} \mathrm{O}_{2}\right)$ Prepared according to general procedure $\mathrm{C}$ using for step 1 : $277 \mathrm{mg}$ 2,4,6-trichlorotriazine ( $1.50 \mathrm{mmol}, 1.00$ equiv.), $282 \mathrm{mg}$ phenol (3.00 mmol, 2.00 equiv.), $523 \mathrm{mg}$ DIPEA (4.05 mmol, 2.70 equiv.); $-35^{\circ} \mathrm{C}$ for $2 \mathrm{~h}$, then r. t. for $70 \mathrm{~h}$; step 2: $161 \mathrm{mg}$ aniline $\left(157 \mathrm{~mm}^{3}, 1.73 \mathrm{mmol}, 1.15\right.$ equiv.), $291 \mathrm{mg}$ DIPEA ( $2.25 \mathrm{mmol}, 1.50$ equiv.); r. t. for $24 \mathrm{~h}$. After the work-up procedure described in general procedure A, $2 \mathrm{~cm}^{3}$ diethyl ether and $5 \mathrm{~cm}^{3} \mathrm{LP}$ were added to induce precipitation and facilitate evaporation to dryness. The crude material was dissolved in a refluxing mixture of $\mathrm{CHCl}_{3} / n$-hexane $\left(3: 2,10 \mathrm{~cm}^{3}\right)$ and more $n$ hexane $\left(15 \mathrm{~cm}^{3}\right)$ was added; after cooling and complete crystallization, the compound $[21,49,50]$ was isolated by filtration, washed with $35 \mathrm{~cm}^{3} \mathrm{LP}$, and dried in vacuo. Yield: $452 \mathrm{mg}$ (85\%); appearance: colorless solid; m.p.: $156-159{ }^{\circ} \mathrm{C} ; \quad R_{f}=0.29$ (hexane/EtOAc, 5:1); ${ }^{1} \mathrm{H}$ NMR (DMSO- $d_{6}, 200 \mathrm{MHz}$ ): $\delta=6.97\left(\mathrm{t}, J_{3}=7.2 \mathrm{~Hz}, 1 \mathrm{H}, \mathrm{H} 4^{\prime}\right.$ ), $7.13\left(\mathrm{t}, J_{3}=7.6 \mathrm{~Hz}, 2 \mathrm{H}, \mathrm{H} 3\right.$ ' \& H5'), 7.22-7.37 (m, 6H), $7.39-7.55(\mathrm{~m}, 6 \mathrm{H}), 10.28(\mathrm{~s}, 1 \mathrm{H}, \mathrm{NH}) \mathrm{ppm} ;{ }^{13} \mathrm{C} \mathrm{NMR}$ (DMSO- $\left.d_{6}, 50 \mathrm{MHz}\right): \delta=120.4$ (d, C2' \& C6'), 121.9 (d, C2" \& C2'" \& C6" \& C6" "), 123.2 (d, C4'), 125.7 (d, C4" \& C4'”), 128.3 (d, C3' \& C5') 129.6 (d, C3" \& C3'” \& C5" \& C5'”), 138.4 (s, C1'), 151.9 (s, C1" \& C1'”), 165.9 (s, C2), $171.8 \& 172.3$ (s, C4 \& C6, rotamers) ppm.

2,4-Diphenoxy-6-(phenylthio)-1,3,5-triazine $\left(7, \mathrm{C}_{21} \mathrm{H}_{15} \mathrm{~N}_{3} \mathrm{O}_{2} \mathrm{~S}\right)$ Prepared according to general procedure $\mathrm{C}$ using for step 1 : $277 \mathrm{mg}$ 2,4,6-trichlorotriazine (1.50 mmol, 1.00 equiv.), $282 \mathrm{mg}$ phenol ( $3.00 \mathrm{mmol}, 2.00$ equiv.), $523 \mathrm{mg}$ DIPEA (4.05 mmol, 2.70 equiv.); $-35^{\circ} \mathrm{C}$ for $2 \mathrm{~h}$, then r. t. for $70 \mathrm{~h}$; step 2: $190 \mathrm{mg}$ thiophenol $\left(176 \mathrm{~mm}^{3}, 1.73 \mathrm{mmol}\right.$, 1.15 equiv.), $291 \mathrm{mg}$ DIPEA ( $2.25 \mathrm{mmol}, 1.50$ equiv.); r. t. for $24 \mathrm{~h}$. After the work-up procedure described in general procedure A, the solvent was evaporated completely and the crude material was dissolved in a refluxing mixture of $\mathrm{CHCl}_{3} / n$-hexane $\left(3: 2, \quad 8.5 \mathrm{~cm}^{3}\right)$ and more $n$-hexane $\left(10 \mathrm{~cm}^{3}\right)$ was added; after cooling and crystallization were completed, the compound was isolated by filtration and dried in vacuo. Yield: $405 \mathrm{mg}$ (72\%); appearance: colorless solid; m.p.: $157-161.5^{\circ} \mathrm{C} ; R_{f}=0.38$ (hexane/EtOAc, 5:1); ${ }^{1} \mathrm{H}$ NMR (DMSO- $\left.d_{6}, 200 \mathrm{MHz}\right): \delta=7.13-7.29(\mathrm{~m}$, $6 \mathrm{H}), 7.30-7.46(\mathrm{~m}, 7 \mathrm{H}), 7.46-7.56(\mathrm{~m}, 2 \mathrm{H}) \mathrm{ppm} ;{ }^{13} \mathrm{C} \mathrm{NMR}$
(DMSO- $\left.d_{6}, 50 \mathrm{MHz}\right): \delta=121.4$ (d, C2' \& C2" \& C6' \& C6"), 125.9 (d, C4' \& C4”), 126.3 (s, C1'”), 129.2 (d, C3" " \& C5'”), 129.5 (d, C3' \& C3" \& C5' \& C5"), 129.7 (d, C4'”), 134.8 (d, C2'” \& C6'”), 151.3 (s, C1' \& C1"), 170.6 (s, C2 \& C4), 184.8 (s, C6) ppm.

\section{Synthesis of 1,1-dimethylethyl (4,6- disubstituted-1,3,5-triazin-2- yl)aminobenzoates-general procedure D}

The reaction was performed in an $8 \mathrm{~cm}^{3}$ glass vial, using a cryo or thermoblock. DIPEA (1.10 equiv.) was added to phenolic compound $\mathrm{R}^{1} \mathrm{PhOH}$ (1.00 equiv.), dissolved in $0.4 \mathrm{~cm}^{3}$ THF. This solution was cooled to $-35^{\circ} \mathrm{C}$ and then added dropwise to a solution of 2,4,6-trichlorotriazine (1.00 equiv.) in $0.7 \mathrm{~cm}^{3} \mathrm{THF}$ at $-35^{\circ} \mathrm{C}$ while stirring. To ensure complete transfer, another $0.4 \mathrm{~cm}^{3}$ of THF was used to flush all phenolic compounds into the reaction mixture. Stirring was continued (step 1) until TLC indicated complete conversion or no significant change in reaction composition. Then, DIPEA (1.60 equiv.) was added to a solution of phenol $\mathrm{R}^{2} \mathrm{PhOH}$ (1.00 equiv.) in $0.4 \mathrm{~cm}^{3} \mathrm{THF}$, and this mixture added to the reaction. To ensure complete transfer, another $0.4 \mathrm{~cm}^{3}$ of THF was used to flush all phenolic compounds into the reaction mixture. Stirring was continued (step 2) and reaction progress was checked by TLC. Then, DIPEA (1.50 equiv.) was added directly to the reaction mixture, followed by 1,1-dimethylethyl 3 -aminobenzoate (1.15 equiv.). The reaction was then continued (step 3) and checked by TLC.

Work-up by general procedure DI $10 \mathrm{~cm}^{3} \mathrm{CH}_{2} \mathrm{Cl}_{2}$ was added to the reaction mixture, followed by $10 \mathrm{~cm}^{3}$ water and the compound was extracted. The layers were separated, the aqueous phase was re-extracted with $5 \mathrm{~cm}^{3}$ $\mathrm{CH}_{2} \mathrm{Cl}_{2}$, and the combined organic fractions concentrated in vacuo. Further work-up and purification procedures are given at the respective examples.

Work-up by general procedure $D 2$ the reaction mixture was taken up with $15 \mathrm{~cm}^{3}$ EtOAc, washed with $1 \mathrm{~N} \mathrm{HCl}_{\mathrm{aq}}$. $\left(2 \times 10 \mathrm{~cm}^{3}\right), 10 \mathrm{~cm}^{3}$ saturated aq. $\mathrm{NaHCO}_{3}$ solution, and $10 \mathrm{~cm}^{3}$ brine. The organic phase was concentrated in vacuo. Further work-up and purification are as stated below. Further work-up and purification procedures are given at the respective examples.

1,1-Dimethylethyl 3-[(4,6-diphenoxy-1,3,5-triazin-2yl)amino]benzoate $\left(8 \mathrm{a}, \mathrm{C}_{26} \mathrm{H}_{24} \mathrm{~N}_{4} \mathrm{O}_{4}\right.$ ) Prepared according to general procedure D using for step 1: $92 \mathrm{mg} \mathrm{2,4,6-}$ trichlorotriazine ( $0.50 \mathrm{mmol}, 1.00$ equiv.), $47 \mathrm{mg}$ phenol ( $0.50 \mathrm{mmol}, 1.00$ equiv.), $71 \mathrm{mg}$ DIPEA ( $0.55 \mathrm{mmol}, 1.10$ equiv.); $-35{ }^{\circ} \mathrm{C}$ for $6 \mathrm{~h}$; step 2: $47 \mathrm{mg}$ phenol ( $0.50 \mathrm{mmol}, 1.00$ equiv.), $103 \mathrm{mg}$ DIPEA (0.80 mmol, 1.60 equiv.); r. t. for $46 \mathrm{~h}$; step 3: $111 \mathrm{mg} \mathrm{1,1-}$ 
dimethylethyl 3-aminobenzoate ( $0.58 \mathrm{mmol}, 1.15$ equiv. $)$, $97 \mathrm{mg}$ DIPEA (0.75 mmol, 1.50 equiv.); r. t. for $43 \mathrm{~h}$. After the work-up procedure described in general procedure $\mathrm{C} 1$, the compound was purified by column chromatography (MPLC, $90 \mathrm{~g}$ silica, $45 \mathrm{~cm}^{3} \mathrm{~min}^{-1}$ flow rate, $\mathrm{CH}_{2} \mathrm{Cl}_{2} / \mathrm{LP}, 1: 5$ with a gradient of $1-25 \%$ EtOAc within $35 \mathrm{~min}$ ) and dried in vacuo. Yield: $167 \mathrm{mg}(73 \%)$; appearance: colorless crystals; m.p.: $208-209{ }^{\circ} \mathrm{C} ; R_{f-}$ $=0.62$ (hexane/EtOAc, 2:1); ${ }^{1} \mathrm{H}$ NMR (DMSO- $d_{6}$, $200 \mathrm{MHz}): \delta=1.51\left(\mathrm{~s}, 9 \mathrm{H},\left(\mathrm{CH}_{3}\right)_{3}\right), 7.14-7.34(\mathrm{~m}, 7 \mathrm{H})$, 7.39-7.55 (m, 5H), $7.75\left(\mathrm{~d}, J_{3}=8.3 \mathrm{~Hz}, 1 \mathrm{H}\right), 8.02(\mathrm{~s}, 1 \mathrm{H}$, H2'), $10.39(\mathrm{~s}, 1 \mathrm{H}, \mathrm{NH}) \mathrm{ppm} ;{ }^{13} \mathrm{C}$ NMR (DMSO- $d_{6}$, $50 \mathrm{MHz}): \delta=27.7\left(\mathrm{q}, \mathrm{C}\left(\mathrm{CH}_{3}\right)_{3}\right), 80.6\left(\mathrm{~s}, \mathrm{C}\left(\mathrm{CH}_{3}\right)_{3}\right), 121.3$ (d, C2'), 121.8 (d, C2" \& C2'” \& C6" \& C6'”), 123.7 (d, C4'), 124.7 (d, C6'), 125.7 (d, C4" \& C4'”), 128.4 (d, C5'), 129.6 (d, C3" \& C3" " \& C5" \& C5"”), 131.7 (s, C3'), 138.7 (s, C1'), 151.8 (s, C1”, C1'”), 164.6 (s, $\mathrm{CO}_{2}$ ), 166.2 (s, C2), $171.8 \& 172.2$ (bs, C4 \& C6, rotamers) ppm.

\section{1,1-Dimethylethyl 3-[[4-(4-chlorophenoxy)-6-phenoxy-} 1,3,5-triazin-2-yl]amino]benzoate $\left(8 \mathrm{~b}, \mathrm{C}_{26} \mathrm{H}_{23} \mathrm{CIN}_{4} \mathrm{O}_{4}\right)$ Prepared according to general procedure $\mathrm{D}$ using for step 1: $92 \mathrm{mg}$ 2,4,6-trichlorotriazine ( $0.50 \mathrm{mmol}, 1.00$ equiv.), $47 \mathrm{mg}$ phenol ( $0.50 \mathrm{mmol}, 1.00$ equiv.), $71 \mathrm{mg}$ DIPEA (0.55 mmol, 1.10 equiv.); $-35{ }^{\circ} \mathrm{C}$ for $6 \mathrm{~h}$; step $2 ; 64 \mathrm{mg}$ 4-chlorophenol ( $0.50 \mathrm{mmol}, 1.00$ equiv.), $103 \mathrm{mg}$ DIPEA (0.80 mmol, 1.60 equiv.); r. t. for $46 \mathrm{~h}$; step 3: $111 \mathrm{mg} \mathrm{1,1-}$ dimethylethyl 3 -aminobenzoate ( $0.58 \mathrm{mmol}, 1.15$ equiv.), $97 \mathrm{mg}$ DIPEA ( $0.75 \mathrm{mmol}, 1.50$ equiv.); $40{ }^{\circ} \mathrm{C}$ for $26 \mathrm{~h}$. After the work-up procedure described in general procedure $\mathrm{C} 1$, the compound was purified by column chromatography (MPLC, $90 \mathrm{~g}$ silica, $45 \mathrm{~cm}^{3} \mathrm{~min}^{-1}$ flow rate, $\mathrm{CH}_{2} \mathrm{Cl}_{2}$ ) and dried in vacuo. Yield: $217 \mathrm{mg}$ (89\%); appearance: off-white solid; m.p.: $172.5-174{ }^{\circ} \mathrm{C} ; R_{f}=0.61$ (hexane/EtOAc, 2:1); ${ }^{1} \mathrm{H}$ NMR (DMSO- $d_{6}, 200 \mathrm{MHz}$ ): $\delta=1.53\left(\mathrm{~s}, 9 \mathrm{H},\left(\mathrm{CH}_{3}\right)_{3}\right), 7.14-7.37(\mathrm{~m}, 6 \mathrm{H}), 7.39-7.58(\mathrm{~m}$, $5 \mathrm{H}), 7.73\left(\mathrm{~d}, J_{3}=8.1 \mathrm{~Hz}, 1 \mathrm{H}\right), 8.04(\mathrm{~s}, 1 \mathrm{H}, \mathrm{H} 2$ ') 10.42 (s, $1 \mathrm{H}, \mathrm{NH}$ ) ppm; ${ }^{13} \mathrm{C}$ NMR (DMSO- $d_{6}, 50 \mathrm{MHz}$ ): $\delta=27.8$ (q, $\left.\mathrm{C}\left(\mathrm{CH}_{3}\right)_{3}\right), 80.7\left(\mathrm{~s}, \mathrm{C}\left(\mathrm{CH}_{3}\right)_{3}\right), 121.3$ (d, $\mathrm{C} 2$ '), 121.8 (d, C2'” \& C6"”), 123.77 (d, C2" \& C6"), 123.85 (d, C4'), 124.8 (d, C6'), 125.7 (d, C4'”), 128.4 (d, C5'), 129.4 (d, C3"* \& C5”*), 129.6 (d, C3" "* \& C5'”*), 129.9 (s, C4”), 131.7 (s, C3'), 138.6 (s, C1'), 150.6 (s, C1"), 151.8 (s, C1'”), 164.6 (s, $\left.\mathrm{CO}_{2}\right), 166.1$ (s, C2), 171.5-172.4 (C4 \& $\mathrm{C} 6$, not resolved) $\mathrm{ppm}$.

\section{1,1-Dimethylethyl 3-[[4-(3-chlorophenoxy)-6-phenoxy-}

1,3,5-triazin-2-yl]amino]benzoate $\left(8 \mathrm{c}, \mathrm{C}_{26} \mathrm{H}_{23} \mathrm{CIN}_{4} \mathrm{O}_{4}\right)$ Prepared according to general procedure $\mathrm{D}$ using for step 1 : $92 \mathrm{mg}$ 2,4,6-trichlorotriazine ( $0.50 \mathrm{mmol}, 1.00$ equiv.), $47 \mathrm{mg}$ phenol (0.50 mmol, 1.00 equiv.), $71 \mathrm{mg}$ DIPEA (0.55 mmol, 1.10 equiv.); $-35{ }^{\circ} \mathrm{C}$ for $6 \mathrm{~h}$; step 2: $64 \mathrm{mg}$ 3-chlorophenol $(0.50 \mathrm{mmol}, 1.00$ equiv., purified by Kugelrohr distillation), $103 \mathrm{mg}$ DIPEA (0.80 mmol, 1.60 equiv.); r. t. for $46 \mathrm{~h}$; step 3: $111 \mathrm{mg}$ 1,1-dimethylethyl 3-aminobenzoate ( $0.58 \mathrm{mmol}, 1.15$ equiv.), $97 \mathrm{mg}$ DIPEA (0.75 mmol, 1.50 equiv.); $40{ }^{\circ} \mathrm{C}$ for $26 \mathrm{~h}$. After the workup procedure described in general procedure $\mathrm{C} 1$, the compound was purified by column chromatography (MPLC, $90 \mathrm{~g}$ silica, $45 \mathrm{~cm}^{3} \mathrm{~min}^{-1}$ flow rate, $\mathrm{LP} / \mathrm{CH}_{2} \mathrm{Cl}_{2} /$ EtOAc, 10: 2:1, with an additional $2 \% \mathrm{Et}_{3} \mathrm{~N}$ ) and dried in vacuo. Yield: $186 \mathrm{mg}$ (76\%); appearance: colorless solid; m.p.: $187.5-190.5{ }^{\circ} \mathrm{C} ; R_{f}=0.64$ (hexane/EtOAc, $2: 1$ ); ${ }^{1} \mathrm{H}$ NMR (DMSO- $\left.d_{6}, 200 \mathrm{MHz}\right): \delta=1.52\left(\mathrm{~s}, 9 \mathrm{H},\left(\mathrm{CH}_{3}\right)_{3}\right)$, 7.13-7.60 (m, 11H), $7.77\left(\mathrm{~d}, J_{3}=8.0 \mathrm{~Hz}, 1 \mathrm{H}\right), 8.04(\mathrm{~s}, 1 \mathrm{H}$, $\mathrm{H}^{\prime}$ ), $10.45(\mathrm{~s}, 1 \mathrm{H}, \mathrm{NH}) \mathrm{ppm} ;{ }^{13} \mathrm{C}$ NMR (DMSO- $d_{6}$, $50 \mathrm{MHz}): \delta=27.7\left(\mathrm{q}, \mathrm{C}\left(\mathrm{CH}_{3}\right)_{3}\right), 80.6\left(\mathrm{~s}, \mathrm{C}\left(\mathrm{CH}_{3}\right)_{3}\right), 120.7$ (d, C6”), 121.3 (d, C2'), 121.7 (d, C2'” \& C6'”), 122.3 (d, C2”), 123.8 (d, C4'), 124.7 (d, C6'), 125.6 (d, C4'”), 125.8 (d, C4”), 128.4 (d, C5'), 129.5 (d, C3'” \& C5'”), 130.8 (d, C5”), 131.7 (s, C3'), 133.4 (s, C3”), 138.6 (s, C1'), 151.8 (s, C1'”), 152.4 (s, C1"), 164.5 (s, $\left.\mathrm{CO}_{2}{ }^{*}\right), 166.1$ (s, C2), 171.2-172.2 (C4 \& C6, not resolved) ppm.

\section{1,1-Dimethylethyl 3-[[4-(2-chlorophenoxy)-6-phenoxy-} 1,3,5-triazin-2-yl]amino]benzoate $\left(8 \mathrm{~d}, \mathrm{C}_{26} \mathrm{H}_{23} \mathrm{CIN}_{4} \mathrm{O}_{4}\right)$ Prepared according to general procedure $\mathrm{D}$ using for step 1: $92 \mathrm{mg}$ 2,4,6-trichlorotriazine ( $0.50 \mathrm{mmol}, 1.00$ equiv.), $47 \mathrm{mg}$ phenol (0.50 mmol, 1.00 equiv.), $71 \mathrm{mg}$ DIPEA (0.55 mmol, 1.10 equiv.); $-35{ }^{\circ} \mathrm{C}$ for $6 \mathrm{~h}$; step 2: $64 \mathrm{mg}$ 2-chlorophenol ( $0.50 \mathrm{mmol}, 1.00$ equiv.), $103 \mathrm{mg}$ DIPEA (0.80 mmol, 1.60 equiv.); r. t. for $46 \mathrm{~h}$; step 3: $111 \mathrm{mg} \mathrm{1,1-}$ dimethylethyl 3 -aminobenzoate $(0.58 \mathrm{mmol}, 1.15$ equiv. $)$, $97 \mathrm{mg}$ DIPEA ( $0.75 \mathrm{mmol}, 1.50$ equiv.); $40{ }^{\circ} \mathrm{C}$ for $26 \mathrm{~h}$. After the work-up procedure described in general procedure $\mathrm{C} 1$, the compound was purified by column chromatography (MPLC, $90 \mathrm{~g}$ silica, $45 \mathrm{~cm}^{3} \mathrm{~min}^{-1}$ flow rate, $\mathrm{LP} / \mathrm{CH}_{2} \mathrm{Cl}_{2} / \mathrm{EtOAc}$, 80: 8: 1, with an additional $2 \% \mathrm{Et}_{3} \mathrm{~N}$ ) and dried in vacuo. Yield: $189 \mathrm{mg}(77 \%)$; appearance: colorless crystals; m.p.: $191-192{ }^{\circ} \mathrm{C} ; R_{f}=0.63$ (hexane/ EtOAc, $2: 1$ ); ${ }^{1} \mathrm{H}$ NMR (DMSO- $\left.d_{6}, 200 \mathrm{MHz}\right): \delta=1.52$ (s, 9H, $\left.\left(\mathrm{CH}_{3}\right)_{3}\right), 7.11-7.56(\mathrm{~m}, 10 \mathrm{H}), 7.56-7.79(\mathrm{~m}, 2 \mathrm{H}), 7.99$ (s, $1 \mathrm{H}, \mathrm{H} 2$ ') 10.48 (s, $1 \mathrm{H}, \mathrm{NH}) \mathrm{ppm} ;{ }^{13} \mathrm{C}$ NMR (DMSO- $d_{6}$, $50 \mathrm{MHz}): \delta=27.7\left(\mathrm{q}, \mathrm{C}\left(\mathrm{CH}_{3}\right)_{3}\right), 80.6\left(\mathrm{~s}, \mathrm{C}\left(\mathrm{CH}_{3}\right)_{3}\right), 121.3$ (d, C2'), 121.7 (d, C2'" \& C6'”), 123.8 (d, C4'), 124.2 (d, C6"), 124.7 (d, C6'), 125.7 (d, C4'”), 125.9 (s, C2”), 127.4 (d, C4"*), 128.4 (d, C5”*), 128.5 (d, C5*), 129.5 (d, C3"” \& C5'”), 130.2 (d, C3"), 131.6 (s, C3'), 138.4 (s, C1'), 147.7 (s, C1"), 151.7 (s, C1"”), 164.5 (s, $\mathrm{CO}_{2}$ ), 166.1 (s, C2), $171.2 \& 171.6 \& 171.8 \& 172.2$ (C4 \& C6, 2 pairs of rotamers) $\mathrm{ppm}$.

1,1-Dimethylethyl 3-[[4-[4-(1,1-dimethylethyl)phenoxy]-6phenoxy-1,3,5-triazin-2-yl]amino]benzoate $\left(8 \mathrm{e}, \mathrm{C}_{30} \mathrm{H}_{32} \mathrm{~N}_{4} \mathrm{O}_{4}\right)$ Prepared according to general procedure $\mathrm{D}$ using for step 1: $92 \mathrm{mg}$ 2,4,6-trichlorotriazine $(0.50 \mathrm{mmol}, 1.00$ equiv.), $47 \mathrm{mg}$ phenol ( $0.50 \mathrm{mmol}, 1.00$ equiv.), $71 \mathrm{mg}$ DIPEA (0.55 mmol, 1.10 equiv.); $-35{ }^{\circ} \mathrm{C}$ for $7 \mathrm{~h}$; step $2: 75 \mathrm{mg}$ 
4-(1,1-dimethylethyl)phenol (0.50 mmol, 1.00 equiv.), $103 \mathrm{mg}$ DIPEA (0.80 mmol, 1.60 equiv.); $40{ }^{\circ} \mathrm{C}$ for $47 \mathrm{~h}$; step 3: $111 \mathrm{mg}$ 1,1-dimethylethyl 3-aminobenzoate (0.58 mmol, 1.15 equiv.), $97 \mathrm{mg}$ DIPEA ( $0.75 \mathrm{mmol}, 1.50$ equiv.); $40{ }^{\circ} \mathrm{C}$ for $43 \mathrm{~h}$. After the work-up procedure described in general procedure $\mathrm{C} 1$, the compound was purified by column chromatography (MPLC, $90 \mathrm{~g}$ silica, $45 \mathrm{~cm}^{3} \mathrm{~min}^{-1}$ flow rate, $\mathrm{LP} / \mathrm{CH}_{2} \mathrm{Cl}_{2}, 5: 1$, with a gradient of EtOAc from 1 to $25 \%$ in $35 \mathrm{~min}$ ) and dried in vacuo. Yield: $170 \mathrm{mg}$ (67\%); appearance: colorless solid; m.p.: $139-141{ }^{\circ} \mathrm{C} ; R_{f}=0.75$ (hexane/EtOAc, 2:1); ${ }^{1} \mathrm{H}$ NMR (DMSO- $\left.d_{6}, 200 \mathrm{MHz}\right): \delta=1.31\left(\mathrm{~s}, 9 \mathrm{H}, \mathrm{C} 4 " \mathrm{C}\left(\mathrm{CH}_{3}\right)_{3}\right)$, 1.52 (s, 9H, $\left.\mathrm{CO}_{2} \mathrm{C}\left(\mathrm{CH}_{3}\right)_{3}\right), 7.10-7.35(\mathrm{~m}, 6 \mathrm{H}), 7.37-7.58$ $(\mathrm{m}, 5 \mathrm{H}), 7.78\left(\mathrm{~d}, J_{3}=7.3 \mathrm{~Hz}, 1 \mathrm{H}\right), 8.03\left(\mathrm{~s}, 1 \mathrm{H}, \mathrm{H} 2{ }^{\prime}\right), 10.39$ $(\mathrm{s}, \quad 1 \mathrm{H}, \mathrm{NH})$ ppm; ${ }^{13} \mathrm{C}$ NMR (DMSO- $\left.d_{6}, 50 \mathrm{MHz}\right)$ : $\delta=27.8\left(\mathrm{q}, \mathrm{CO}_{2} \mathrm{C}\left(\mathrm{CH}_{3}\right)_{3}\right), 31.3$ (q, $\left.\mathrm{C} 4 " \mathrm{C}\left(\mathrm{CH}_{3}\right)_{3}\right), 34.3$ (s, $\mathrm{C} 4$ " $\left.\mathrm{C}\left(\mathrm{CH}_{3}\right)_{3}\right), 80.7\left(\mathrm{~s}, \mathrm{CO}_{2} \mathrm{C}\left(\mathrm{CH}_{3}\right)_{3}\right), 121.2$ (d, $\mathrm{C} 2$ " \& C6"*), 121.3 (d, C2'*), 121.8 (d, C2'” \& C6"”), 123.7 (d, C4'), 124.7 (d, C6'), 125.7 (d, C4'”), 126.2 (d, C3" \& C5”), 128.4 (d, C5'), 129.6 (d, C3" " \& C5'”), 131.6 (s, C3'), 138.7 (s, C1'), 148.0 (s, C4"*), 149.5 (s, C1"*), 151.8 (s, C1'”), 164.6 (s, $\mathrm{CO}_{2}$ ), 166.2 (s, C2), 171.4-172.3 (C4 \& C6, not resolved) ppm.

\section{1,1-Dimethylethyl 3-[[4-(4-methoxyphenoxy)-6-phenoxy-} 1,3,5-triazin-2-yl]amino]benzoate $\left(8 f, \quad \mathrm{C}_{27} \mathrm{H}_{26} \mathrm{~N}_{4} \mathrm{O}_{5}\right)$ Prepared according to general procedure $\mathrm{D}$ using for step 1 : $92 \mathrm{mg}$ 2,4,6-trichlorotriazine ( $0.50 \mathrm{mmol}, 1.00$ equiv.), $47 \mathrm{mg}$ phenol ( $0.50 \mathrm{mmol}, 1.00$ equiv.), $71 \mathrm{mg}$ DIPEA ( $0.55 \mathrm{mmol}, 1.10$ equiv.); $-35{ }^{\circ} \mathrm{C}$ for $7 \mathrm{~h}$; step 2: $62 \mathrm{mg}$ 4-methoxyphenol (0.50 mmol, 1.00 equiv.), $103 \mathrm{mg}$ DIPEA (0.80 mmol, 1.60 equiv.); $40{ }^{\circ} \mathrm{C}$ for $47 \mathrm{~h}$; step 3 : $111 \mathrm{mg}$ 1,1-dimethylethyl 3-aminobenzoate $(0.58 \mathrm{mmol}$, 1.15 equiv.), $97 \mathrm{mg}$ DIPEA ( $0.75 \mathrm{mmol}, 1.50$ equiv.); $40{ }^{\circ} \mathrm{C}$ for $43 \mathrm{~h}$. After the work-up procedure described in general procedure $\mathrm{C} 2,2 \mathrm{~cm}^{3}$ diethyl ether and $3 \mathrm{~cm}^{3} \mathrm{LP}$ was added and the oily material was sonicated to induce precipitation. After cooling to $0{ }^{\circ} \mathrm{C}$ for $2 \mathrm{~h}$, the precipitate was collected, and $10 \mathrm{~cm}^{3} \mathrm{LP}$ was added to the supernatant to induce crystallization of a second fraction which was collected by centrifugation. The combined fractions were purified by column chromatography (MPLC, silica, $45 \mathrm{~cm}^{3}$ $\min ^{-1}$ flow rate, $\mathrm{LP}$ with a gradient of $\mathrm{Et}_{2} \mathrm{O}$ from 15 to $75 \%$ in $80 \mathrm{~min}$ ) and dried in vacuo. Yield: $167 \mathrm{mg}(68 \%)$; appearance: colorless crystals; m.p.: $68-70{ }^{\circ} \mathrm{C} ; R_{f}=0.56$ (hexane/EtOAc, 2:1); ${ }^{1} \mathrm{H}$ NMR (DMSO- $d_{6}, 200 \mathrm{MHz}$ ): $\delta=1.52\left(\mathrm{~s}, 9 \mathrm{H},\left(\mathrm{CH}_{3}\right)_{3}\right), 3.77\left(\mathrm{~s}, 3 \mathrm{H}, \mathrm{OCH}_{3}\right), 6.97(\mathrm{~d}$, $J_{3}=9.1 \mathrm{~Hz}, 2 \mathrm{H}, \mathrm{H} 3$ " \& H5"), 7.12-7.34 (m, 6H), $7.38-7.58(\mathrm{~m}, 3 \mathrm{H}), 7.77\left(\mathrm{~d}, J_{3}=7.5 \mathrm{~Hz}, 1 \mathrm{H}\right), 8.04(\mathrm{~s}, 1 \mathrm{H}$, $\left.\mathrm{H} 2^{\prime}\right), 10.37$ (s, 1H, NH) ppm; ${ }^{13} \mathrm{C}$ NMR (DMSO- $d_{6}$, $50 \mathrm{MHz}): \delta=27.8\left(\mathrm{q}, \mathrm{C}\left(\mathrm{CH}_{3}\right)_{3}\right), 55.5\left(\mathrm{q}, \mathrm{OCH}_{3}\right), 80.7$ (s, $\left.\mathrm{C}\left(\mathrm{CH}_{3}\right)_{3}\right), 114.5$ (d, C3" \& C5"), 121.2 (d, C2'), 121.8 (d, C2'” \& C6"”), 122.6 (d, C2" \& C6"), 123.8 (d, C4'), 124.8 (d, C6'), 125.7 (d, C4'”), 128.5 (d, C5'), 129.6 (d, C3'” \& C5'”), 131.7 (s, C3'), 138.7 (s, C1'), 145.2 (s, C1"), 151.8 (s, C1'”), 156.9 (s, C4"), 164.6 (s, $\mathrm{CO}_{2}$ ), 166.2 (s, C2), 171.2-172.4 (C4 \& C6, not resolved) ppm.

1,1-Dimethylethyl 3-[[4-(4-cyanophenoxy)-6-phenoxy-1,3,5triazin-2-yl]amino]benzoate $\left(8 \mathrm{~g}, \quad \mathrm{C}_{27} \mathrm{H}_{23} \mathrm{~N}_{5} \mathrm{O}_{4}\right)$ Prepared according to general procedure D using for step 1: $92 \mathrm{mg}$ 2,4,6-trichlorotriazine $(0.50 \mathrm{mmol}, 1.00$ equiv. $), 47 \mathrm{mg}$ phenol $(0.50 \mathrm{mmol}, \quad 1.00$ equiv. $), \quad 71 \mathrm{mg}$ DIPEA ( $0.55 \mathrm{mmol}, 1.10$ equiv.); $-35{ }^{\circ} \mathrm{C}$ for $7 \mathrm{~h}$; step 2: $60 \mathrm{mg}$ 4-hydroxybenzonitrile $(0.50 \mathrm{mmol}, 1.00$ equiv. $), 103 \mathrm{mg}$ DIPEA (0.80 mmol, 1.60 equiv.); $40{ }^{\circ} \mathrm{C}$ for $47 \mathrm{~h}$; step 3: $111 \mathrm{mg}$ 1,1-dimethylethyl 3-aminobenzoate $(0.58 \mathrm{mmol}$, 1.15 equiv.), $97 \mathrm{mg}$ DIPEA (0.75 mmol, 1.50 equiv.); $40{ }^{\circ} \mathrm{C}$ for $43 \mathrm{~h}$. After the work-up procedure described in general procedure $\mathrm{C} 2$, the compound was purified by column chromatography (MPLC, silica, $45 \mathrm{~cm}^{3} \mathrm{~min}^{-1}$ flow rate, liquid appl. with $\mathrm{CH}_{2} \mathrm{Cl}_{2}$, $\mathrm{LP}$ with a gradient of EtOAc from 4 to $76 \%$ within $80 \mathrm{~min}$ ) and dried in vacuo. Yield: $81 \mathrm{mg}$ (33\%); appearance: white solid; m.p.: 176.5-178.5 ${ }^{\circ} \mathrm{C} ; R_{f}=0.49$ (hexane/EtOAc, 2:1); ${ }^{1} \mathrm{H}$ NMR (DMSO- $\left.d_{6}, \quad 200 \mathrm{MHz}\right): \quad \delta=1.53 \quad\left(\mathrm{~s}, \quad 9 \mathrm{H}, \quad\left(\mathrm{CH}_{3}\right)_{3}\right)$, 7.17-7.34 (m, 4H), 7.38-7.58 (m, 5H), 7.71 (bs, 1H), 7.94 (d, $J_{3}=8.5 \mathrm{~Hz}, 2 \mathrm{H}, \mathrm{H} 3$ ” \& H5”), 8.04 (s, 1H, H2'), 10.46 (s, $1 \mathrm{H}, \mathrm{NH}) \mathrm{ppm} ;{ }^{13} \mathrm{C}$ NMR (DMSO- $d_{6}, 50 \mathrm{MHz}$ ): $\delta=27.8\left(\mathrm{q}, \mathrm{C}\left(\mathrm{CH}_{3}\right)_{3}\right), 80.7\left(\mathrm{~s}, \mathrm{C}\left(\mathrm{CH}_{3}\right)_{3}\right), 108.5$ (s, C4"), 118.5 (s, CN), 121.3 (d, C2'), 121.8 (d, C2'" \& C6'"), 123.2 (d, C2" \& C6"), 123.9 (d, C4**), 124.1 (d, C6**), 125.8 (d, C4'”), 128.5 (d, C5'), 129.6 (d, C3'” \& C5'”), 131.7 (s, C3'), 134.1 (d, C3" \& C5"), 138.5 (s, C1'), 151.7 (s, C1'”), 155.2 (s, C1"), $164.5\left(\mathrm{~s}, \mathrm{CO}_{2}\right), 166.2$ (s, C2) ppm, C4 \& C6 not visible.

1,1-Dimethylethyl 3-[[4-(3-nitrophenoxy)-6-phenoxy-1,3,5triazin-2-yl]amino]benzoate $\left(8 \mathrm{~h}, \mathrm{C}_{26} \mathrm{H}_{23} \mathrm{~N}_{5} \mathrm{O}_{6}\right)$ Prepared according to general procedure D using for step 1: $92 \mathrm{mg}$ 2,4,6-trichlorotriazine ( $0.50 \mathrm{mmol}, 1.00$ equiv.), $47 \mathrm{mg}$ phenol (0.50 mmol, 1.00 equiv.), $71 \mathrm{mg}$ DIPEA (0.55 mmol, 1.10 equiv.); $-35{ }^{\circ} \mathrm{C}$ for $7 \mathrm{~h}$; step 2: $70 \mathrm{mg}$ 3-nitrophenol (0.50 mmol, 1.00 equiv.), $103 \mathrm{mg}$ DIPEA (0.80 mmol, 1.60 equiv.); $40{ }^{\circ} \mathrm{C}$ for $47 \mathrm{~h}$; step 3: $111 \mathrm{mg}$ 1,1-dimethylethyl 3-aminobenzoate $\quad(0.58 \mathrm{mmol}, \quad 1.15$ equiv.), $97 \mathrm{mg}$ DIPEA ( $0.75 \mathrm{mmol}, 1.50$ equiv.); $40{ }^{\circ} \mathrm{C}$ for $43 \mathrm{~h}$. After the work-up procedure described in general procedure $\mathrm{C} 2,1 \mathrm{~cm}^{3}$ EtOAc and $9 \mathrm{~cm}^{3} \mathrm{LP}$ were added and the oily material sonicated to induce precipitation. The supernatant was removed by centrifugation and the compound purified by preparative TLC (LP: EtOAc, 3: 1) and dried in vacuo. Yield: $83 \mathrm{mg}$ (33\%); appearance: yellowish solid; m.p.: $137-148{ }^{\circ} \mathrm{C} ; R_{f}=0.47$ (hexane/EtOAc, 2:1); ${ }^{1} \mathrm{H}$ NMR (DMSO- $\left.d_{6}, 200 \mathrm{MHz}\right): \delta=1.51\left(\mathrm{~s}, 9 \mathrm{H},\left(\mathrm{CH}_{3}\right)_{3}\right)$, 7.16-7.32 (m, 4H), 7.35-7.57 (m, 3H), 7.62-7.84 (m, 3H), $8.04\left(\mathrm{~s}, 1 \mathrm{H}, \mathrm{H} 2\right.$ '), $8.15\left(\mathrm{dt}, J_{3}=7.2 \mathrm{~Hz}, J_{4}=2.0 \mathrm{~Hz}, 1 \mathrm{H}\right)$, 
8.22 (s, 1H, H2"), 10.48 (s, 1H, NH) ppm; ${ }^{13} \mathrm{C}$ NMR (DMSO- $\left.d_{6}, 50 \mathrm{MHz}\right): \delta=27.7\left(\mathrm{q}, \mathrm{C}\left(\mathrm{CH}_{3}\right)_{3}\right), 80.7$ (s, $\left.\mathrm{C}\left(\mathrm{CH}_{3}\right)_{3}\right), 117.4$ (d, C2"), 120.7 (d, C4”), 121.2 (d, C2'), 121.7 (d, C2'” \& C6'”), 124.0 (d, C4'), 124.8 (d, C6'), 125.7 (d, C4"”), 128.5 (d, C6”*), 128.8 (d, C5'*), 129.6 (d, C3"” \& C5'”), 130.8 (d, C5”), 131.7 (s, C3'), 138.5 (s, C1'), 148.4 (s, C3”), 151.7 (s, C1'”*), 152.0 (s, C1”*), $164.5\left(\mathrm{~s}, \mathrm{CO}_{2}\right), 166.1$ (s, C2) ppm, $\mathrm{C} 4$ \& $\mathrm{C} 6$ not visible.

\section{1,1-Dimethylethyl 3-[[4-(4-formyl-2-methoxyphenoxy)-6- phenoxy-1,3,5-triazin-2-yl]amino]benzoate $\left(8 \mathrm{i}, \mathrm{C}_{28} \mathrm{H}_{26} \mathrm{~N}_{4} \mathrm{O}_{6}\right)$ -}

Prepared according to general procedure $\mathrm{D}$ using for step 1: $92 \mathrm{mg}$ 2,4,6-trichlorotriazine ( $0.50 \mathrm{mmol}, 1.00$ equiv.), $47 \mathrm{mg}$ phenol $(0.50 \mathrm{mmol}, 1.00$ equiv. $), 71 \mathrm{mg}$ DIPEA (0.55 mmol, 1.10 equiv.); $-35{ }^{\circ} \mathrm{C}$ for $7 \mathrm{~h}$; step $2: 76 \mathrm{mg}$ vanillin $(0.50 \mathrm{mmol}, 1.00$ equiv. $), 103 \mathrm{mg}$ DIPEA (0.80 mmol, 1.60 equiv.); $40{ }^{\circ} \mathrm{C}$ for $47 \mathrm{~h}$; step 3: $111 \mathrm{mg}$ 1,1-dimethylethyl 3 -aminobenzoate $(0.58 \mathrm{mmol}, \quad 1.15$ equiv.), $97 \mathrm{mg}$ DIPEA ( $0.75 \mathrm{mmol}, 1.50$ equiv.); $40{ }^{\circ} \mathrm{C}$ for $43 \mathrm{~h}$. After the work-up procedure described in general procedure $\mathrm{C} 2$, the compound was purified by column chromatography (MPLC, $90 \mathrm{~g}$ silica, $45 \mathrm{~cm}^{3} \mathrm{~min}^{-1}$ flow rate, LP with a gradient of EtOAc from 1 to $40 \%$ within $1 \mathrm{~h})$ and dried in vacuo. Yield: $141 \mathrm{mg}(55 \%)$; appearance: colorless solid; m.p.: $76-79{ }^{\circ} \mathrm{C} ; R_{f}=0.31$ (hexane/EtOAc, 2:1); ${ }^{1} \mathrm{H}$ NMR (DMSO- $\left.d_{6}, 200 \mathrm{MHz}\right): \delta=1.51$ (s, 9H, $\left.\left(\mathrm{CH}_{3}\right)_{3}\right), 3.86\left(\mathrm{~s}, 3 \mathrm{H}, \mathrm{OCH}_{3}\right), 7.11-7.33(\mathrm{~m}, 4 \mathrm{H}), 7.36-7.55$ (m, 4H), 7.56-7.78 (m, 3H), 8.00 (s, 1H, H2'), 10.00 (s, $1 \mathrm{H}, \mathrm{CHO}), 10.43$ (s, $1 \mathrm{H}, \mathrm{NH}) \mathrm{ppm} ;{ }^{13} \mathrm{C}$ NMR (DMSO- $d_{6}$, $50 \mathrm{MHz}): \delta=27.8\left(\mathrm{q}, \mathrm{C}\left(\mathrm{CH}_{3}\right)_{3}\right), 56.2\left(\mathrm{q}, \mathrm{OCH}_{3}\right), 80.8$ (s, $\left.\mathrm{C}\left(\mathrm{CH}_{3}\right)_{3}\right), 112.3$ (d, $\mathrm{C} 3$ ") $), 121.3$ (d, C2'), 121.8 (d, $\mathrm{C} 2$ '" \& C6" "), 123.6 (d, C5"*), 123.8 (d, C4'*), 123.9 (d, C6"*), 124.7 (d, C6'), 125.8 (d, C4'”), 128.5 (d, C5'), 129.6 (d, C3'” \& C5'”), 131.7 (s, C3'), 135.1 (s, C4"), 138.6 (s, C1'), 145.3 (s, C1"*), 151.70 (s, C2"*) 151.74 (s, C1"”*), 164.6 (s, $\mathrm{CO}_{2}$ ), 166.1 (s, C2), 192.2 (d, CHO) ppm, C4 \& C6 not visible.

\section{1,1-Dimethylethyl 3-[[4-(4-formyl-2-methoxyphenoxy)-6-(4-} methoxyphenoxy)-1,3,5-triazin-2-yl]amino]benzoate (8j, $\left.\mathrm{C}_{29} \mathrm{H}_{28} \mathrm{~N}_{4} \mathrm{O}_{7}\right)$ Prepared according to general procedure D using for step 1: $92 \mathrm{mg} \mathrm{2,4,6-trichlorotriazine}(0.50 \mathrm{mmol}$, 1.00 equiv.), $62 \mathrm{mg}$ 4-methoxyphenol $(0.50 \mathrm{mmol}, 1.00$ equiv.), $71 \mathrm{mg}$ DIPEA ( $0.55 \mathrm{mmol}, 1.10$ equiv.); $-35^{\circ} \mathrm{C}$ for $6 \mathrm{~h}$; step 2: $76 \mathrm{mg}$ vanillin $(0.50 \mathrm{mmol}, 1.00$ equiv. $)$, $103 \mathrm{mg}$ DIPEA ( $0.80 \mathrm{mmol}, 1.60$ equiv.); $40{ }^{\circ} \mathrm{C}$ for $45 \mathrm{~h}$; step 3: $111 \mathrm{mg}$ 1,1-dimethylethyl 3-aminobenzoate (0.58 mmol, 1.15 equiv.), $97 \mathrm{mg}$ DIPEA ( $0.75 \mathrm{mmol}, 1.50$ equiv.); $40{ }^{\circ} \mathrm{C}$ for $22 \mathrm{~h}$. After the work-up procedure described in general procedure $\mathrm{C} 2$, the compound was purified by column chromatography (MPLC, $105 \mathrm{~g}$ silica, $50 \mathrm{~cm}^{3} \mathrm{~min}^{-1}$ flow rate, LP with a gradient of EtOAc from 20 to $40 \%$ within $30 \mathrm{~min}$, then to $100 \%$ within $15 \mathrm{~min}$ ) and dried in vacuo. Yield: $162 \mathrm{mg} \mathrm{(59 \% );} \mathrm{appearance:}$ colorless solid; m.p.: $88-106{ }^{\circ} \mathrm{C} ; \quad R_{f}=0.30$ (hexane/ EtOAc, $2: 1) ;{ }^{1} \mathrm{H}$ NMR (DMSO- $\left.d_{6}, 200 \mathrm{MHz}\right): \delta=1.52(\mathrm{~s}$, $\left.9 \mathrm{H},\left(\mathrm{CH}_{3}\right)_{3}\right), 3.76\left(\mathrm{~s}, 3 \mathrm{H}, \mathrm{C} 4{ }^{\prime}, \mathrm{OCH}_{3}\right), 3.86(\mathrm{~s}, 3 \mathrm{H}$, $\left.\mathrm{C} 2^{\prime} \mathrm{OCH}_{3}\right), 6.96\left(\mathrm{~d}, J_{3}=9.0 \mathrm{~Hz}, 2 \mathrm{H}, \mathrm{H} 3\right.$ '" \& H5'”), 7.10-7.31 (m, 3H), 7.44-7.86 (m, 5H), 8.02 (s, 1H, H2'), 10.01 (s, 1H, CHO), 10.42 (s, 1H, NH) ppm; ${ }^{13} \mathrm{C}$ NMR $\left(\right.$ DMSO- $\left.d_{6}, 50 \mathrm{MHz}\right): \delta=27.7\left(\mathrm{q}, \mathrm{C}\left(\mathrm{CH}_{3}\right)_{3}\right), 55.4$ (q, C4'” $\left.\mathrm{OCH}_{3}\right), 56.1$ (q, C2" $\left.{ }^{\prime} \mathrm{OCH}_{3}\right), 80.7\left(\mathrm{~s}, \mathrm{C}\left(\mathrm{CH}_{3}\right)_{3}\right), 112.2$ (d, C3"), 114.5 (d, C3'” \& C5'”), 121.2 (d, C2'), 122.6 (d, C2'" \& C6"”), 123.6 (d, C5"*), 123.8 (d, C4'*), 123.9 (d, C6”*), 124.6 (d, C6'), 128.5 (d, C5'), 131.7 (s, C3'), 135.0 (s, C4"), 138.6 (s, C1'), 145.2 (s, C1"*), 145.3 (s, C1'”*), 151.8 (s, C2"), 156.9 (s, C4"”), 164.6 (s, $\mathrm{CO}_{2}$ ), 166.1 (s, C2), 192.1 (d, CHO) ppm, C4 \& C6 not visible.

\section{1,1-Dimethylethyl 3-[[4-(4-cyanophenoxy)-6-(4-} methoxyphenoxy)-1,3,5-triazin-2-yl]amino]benzoate (8k, $\mathrm{C}_{28} \mathrm{H}_{25} \mathrm{~N}_{5} \mathrm{O}_{5}$ ) Prepared according to general procedure D using for step 1: $92 \mathrm{mg} \mathrm{2,4,6-trichlorotriazine}(0.50 \mathrm{mmol}$, 1.00 equiv.), $62 \mathrm{mg}$ 4-methoxyphenol $(0.50 \mathrm{mmol}, 1.00$ equiv.), $71 \mathrm{mg}$ DIPEA ( $0.55 \mathrm{mmol}, 1.10$ equiv.); $-35^{\circ} \mathrm{C}$ for $7 \mathrm{~h}$; step 2: $60 \mathrm{mg}$ 4-hydroxybenzonitrile $(0.50 \mathrm{mmol}$, 1.00 equiv.), $103 \mathrm{mg}$ DIPEA ( $0.80 \mathrm{mmol}, 1.60$ equiv.); $40{ }^{\circ} \mathrm{C}$ for $47 \mathrm{~h}$; step 3: $111 \mathrm{mg}$ 1,1-dimethylethyl 3 -aminobenzoate ( $0.58 \mathrm{mmol}, 1.15$ equiv.), $97 \mathrm{mg}$ DIPEA ( $0.75 \mathrm{mmol}, 1.50$ equiv.); $40{ }^{\circ} \mathrm{C}$ for $43 \mathrm{~h}$. After the workup procedure described in general procedure $\mathrm{C} 2$, the compound was purified by column chromatography (MPLC, $90 \mathrm{~g}$ silica, $45 \mathrm{~cm}^{3} \mathrm{~min}^{-1}$ flow rate, LP with a gradient of EtOAc from 10 to $40 \%$ within $1 \mathrm{~h}$ ) and dried in vacuo. Yield: $145 \mathrm{mg}$ (57\%); appearance: colorless solid; m.p.: $161-164{ }^{\circ} \mathrm{C} ; R_{f}=0.43$ (hexane/EtOAc, 2:1); ${ }^{1} \mathrm{H}$ NMR (DMSO- $\left.d_{6}, 200 \mathrm{MHz}\right): \delta=1.53\left(\mathrm{~s}, 9 \mathrm{H},\left(\mathrm{CH}_{3}\right)_{3}\right)$, $3.77\left(\mathrm{~s}, 3 \mathrm{H}, \mathrm{OCH}_{3}\right), 6.98\left(\mathrm{~d}, J_{3}=9.0 \mathrm{~Hz}, 2 \mathrm{H}, \mathrm{H} 3\right.$ " " \& H5'”), 7.11-7.32 (m, 3H), 7.46-7.59 (m, 3H), 7.61-7.84 $(\mathrm{m}, 1 \mathrm{H}), 7.94\left(\mathrm{~d}, J_{3}=8.4 \mathrm{~Hz}, 2 \mathrm{H}, \mathrm{H} 3\right.$ ” \& H5”), 8.05 (s, $1 \mathrm{H}, \mathrm{H} 2$ ') 10.45 (s, 1H, NH) ppm; ${ }^{13} \mathrm{C}$ NMR (DMSO- $d_{6}$, $50 \mathrm{MHz}): \delta=27.8\left(\mathrm{q}, \mathrm{C}\left(\mathrm{CH}_{3}\right)_{3}\right), 55.6\left(\mathrm{q}, \mathrm{C} 4\right.$ '” $\left.\mathrm{OCH}_{3}\right)$, 80.8 (s, $\left.\mathrm{C}\left(\mathrm{CH}_{3}\right)_{3}\right), 108.5$ (s, C4"), 114.5 (d, C3'” \& C5'”), 118.4 (s, CN), 121.3 (d, C2'), 122.6 (d, C2'” \& C6'”), 123.2 (d, C2" \& C6"), 124.0 (d, C4'), 124.9 (d, C6'), 128.5 (d, C5'), 131.7 (s, C3'), 134.1 (d, C3" \& C5”), 138.6 (s, C1'), 145.2 (s, C1'”), 155.2 (s, C1"), 156.9 (s, C4'”), 164.6 (s, $\mathrm{CO}_{2}$ ), 166.1 (s, C2) ppm, C4 \& C6 not visible.

\section{Synthesis of (4,6-disubstituted-1,3,5-triazin-2- yl)aminophenyl methyl alcohols-general procedure $E$}

The reaction was performed using an $8 \mathrm{~cm}^{3}$ vial, using a mixture of $\mathrm{MeOH} /$ liq. $\mathrm{N}_{2}$ for cooling. A vial was charged with alkyl aminobenzoate 5a-5g (1.00 equiv.) and sealed, 
and the atmosphere changed to argon using standard Schlenk technique. Anhydrous $\mathrm{CH}_{2} \mathrm{Cl}_{2}\left(1.0 \mathrm{~cm}^{3}\right)$ was added via syringe, stirred at r. t. until the ester was dissolved, and then cooled in a MeOH/liq. $\mathrm{N}_{2}$ bath. Subsequently, a solution of diisobutylaluminium hydride (DIBAL-H, $0.86 \mathrm{mmol} \mathrm{cm}^{-3}$ ) in $n$-hexane was added slowly. The reaction mixture was stirred until TLC indicated complete conversion (in some cases, more DIBAL-H solution was added in the course of the reaction). Then, the reaction was quenched by adding $1.0 \mathrm{~cm}^{3} 1 \mathrm{M} \mathrm{HCl}_{\mathrm{aq}}$, stirred for $5 \mathrm{~min}$, and then removed from the cooling bath, followed by the addition of $10 \mathrm{~cm}^{3} \mathrm{H}_{2} \mathrm{O}$. After extraction with $\mathrm{CH}_{2} \mathrm{Cl}_{2}$ $\left(10 \mathrm{~cm}^{3}\right.$, then $\left.2 \times 5 \mathrm{~cm}^{3}\right)$, the solvent was removed in vacuo and the compound was purified as stated below.

\section{[4-[(4,6-Diphenoxy-1,3,5-triazin-2-yl)amino]phenyl]metha-}

nol ( $9 \mathrm{a}, \mathrm{C}_{22} \mathrm{H}_{18} \mathrm{~N}_{4} \mathrm{O}_{3}$ ) Prepared according to general procedure $\mathrm{E}$ using $32 \mathrm{mg}$ ethyl aminobenzoate $\mathbf{5 a}$ (0.075 mmol, $\quad 1.00$ equiv.), $0.33 \mathrm{~cm}^{3} \quad$ DIBAL-H ( $0.285 \mathrm{mmol}, 3.80$ equiv.); $-70{ }^{\circ} \mathrm{C}$ for $2.25 \mathrm{~h}$; then more DIBAL-H $\left(0.05 \mathrm{~cm}^{3}, 0.045 \mathrm{mmol}, 0.60\right.$ equiv.); $-70{ }^{\circ} \mathrm{C}$ for $1 \mathrm{~h}$. After work-up as described in general procedure $\mathrm{E}$, the compound was dried in vacuo and did not require further purification. Yield: $29 \mathrm{mg}$ (quant.); appearance: colorless powder; m.p.: $152.5-154{ }^{\circ} \mathrm{C} ; R_{f}=0.36$ (hexane/ EtOAc, $1: 1) ;{ }^{1} \mathrm{H}$ NMR (DMSO- $d_{6}, 200 \mathrm{MHz}$ ): $\delta=4.37$ (d, $\left.J_{3}=5.6 \mathrm{~Hz}, 2 \mathrm{H}, \mathrm{CH}_{2}\right), 5.07\left(\mathrm{t}, J_{3}=5.6 \mathrm{~Hz}, 1 \mathrm{H}, \mathrm{OH}\right), 7.04$ (d, $J_{3}=8.5 \mathrm{~Hz}, 2 \mathrm{H}, \mathrm{H} 3$ ' \& H5'), 7.21-7.54 (m, 12H), $10.20(\mathrm{~s}, 1 \mathrm{H}, \mathrm{NH}) \mathrm{ppm} ;{ }^{13} \mathrm{C}$ NMR (DMSO- $d_{6}, 50 \mathrm{MHz}$ ): $\delta=62.5\left(\mathrm{t}, \mathrm{OCH}_{2}\right), 120.0(\mathrm{~d}, \mathrm{C} 2$ ' \& C6'), 121.9 (d, C2" \& C2'" \& C6" \& C6" "), 125.7 (d, C4" \& C4"”), 126.6 (d, C3' \& C5'), 129.6 (d, C3" \& C3'" \& C5" \& C5'”), 137.0 (s, C4'*), 137.4 (s, C1'*), 151.9 (s, C1" \& C1'”), 165.8 (s, C2), $171.7 \& 172.4$ (s, C4 \& C6, rotamers) ppm.

\section{[3-[(4,6-Diphenoxy-1,3,5-triazin-2-yl)amino]phenyl]metha-}

nol (9b, $\mathrm{C}_{22} \mathrm{H}_{18} \mathrm{~N}_{4} \mathrm{O}_{3}$ ) Prepared according to general procedure $\mathrm{E}$ using $31 \mathrm{mg}$ methyl aminobenzoate $\mathbf{5 b}$ (0.075 mmol, $\quad 1.00$ equiv.), $0.33 \mathrm{~cm}^{3} \quad$ DIBAL-H (0.285 mmol, 3.80 equiv.); $-70{ }^{\circ} \mathrm{C}$ for $1 \mathrm{~h}$. After work-up as described in general procedure $\mathrm{E}$, the compound was dried in vacuo and did not require further purification. Yield: $29 \mathrm{mg}$ (quant.); appearance: colorless powder; m.p.: $49-52{ }^{\circ} \mathrm{C} ; \quad R_{f}=0.36 \quad$ (hexane/EtOAc, $1: 1$ ); ${ }^{1} \mathrm{H} \quad \mathrm{NMR}$ (DMSO- $\left.d_{6}, 200 \mathrm{MHz}\right): \delta=4.27\left(\mathrm{~d}, J_{3}=5.7 \mathrm{~Hz}, 2 \mathrm{H}\right.$, $\left.\mathrm{OCH}_{2}\right), \quad 5.09\left(\mathrm{t}, \quad J_{3}=5.7 \mathrm{~Hz}, \quad 1 \mathrm{H}, \quad \mathrm{OH}\right), \quad 6.95 \quad(\mathrm{~d}$, $\left.J_{3}=7.3 \mathrm{~Hz}, 1 \mathrm{H}\right), 7.07$ (t, $J_{3}=7.7 \mathrm{~Hz}, 1 \mathrm{H}, \mathrm{H} 5$ '), 7.20-7.38 $(\mathrm{m}, 8 \mathrm{H}), 7.39-7.53(\mathrm{~m}, 4 \mathrm{H}), 10.22(\mathrm{~s}, 1 \mathrm{H}, \mathrm{NH}) \mathrm{ppm} ;{ }^{13} \mathrm{C}$ NMR (DMSO- $\left.d_{6}, 50 \mathrm{MHz}\right): \delta=62.8\left(\mathrm{t}, \mathrm{CH}_{2}\right), 118.3(\mathrm{~d}$, C2'*), 118.8 (d, C6'*), 121.3 (d, C4'*), 121.8 (d, C2" \& C2" " \& C6" \& C6" "), 125.6 (d, C4" \& C4"”), 127.9 (d, C5'), 129.5 (d, C3" \& C3" " \& C5" \& C5'”), 138.0 (s, C1'), 142.9 (s, C3'), 151.9 (s, C1" \& C1'”), 166.0 (s, C2) ppm, C4 \& C6 not visible.
[3-[[4-(4-Chlorophenoxy)-6-phenoxy-1,3,5-triazin-2-yl]amino]phenyl]methanol $\left(9 c, \mathrm{C}_{22} \mathrm{H}_{17} \mathrm{ClN}_{4} \mathrm{O}_{3}\right)$ Prepared according to general procedure $\mathrm{E}$ using $34 \mathrm{mg}$ methyl aminobenzoate 5 c $(0.075 \mathrm{mmol}, 1.00$ equiv. $), 0.35 \mathrm{~cm}^{3}$ DIBAL-H ( $0.30 \mathrm{mmol}, 4.00$ equiv. $) ;-70{ }^{\circ} \mathrm{C}$ for $2.25 \mathrm{~h}$; then more DIBAL-H $\left(0.05 \mathrm{~cm}^{3}, 0.045 \mathrm{mmol}, 0.60\right.$ equiv.); $-70{ }^{\circ} \mathrm{C}$ for $1 \mathrm{~h}$. After work-up as described in general procedure $\mathrm{E}$, the compound was re-crystallized from $1.5 \mathrm{~cm}^{3} \mathrm{CHCl}_{3}$ and $10 \mathrm{~cm}^{3} n$-hexane and dried in vacuo. Yield: $28 \mathrm{mg}$ (89\%); appearance: colorless solid; m.p.: $110.5-114{ }^{\circ} \mathrm{C} ; R_{f}=0.39$ (hexane/EtOAc, $1: 1$ ); ${ }^{1} \mathrm{H}$ NMR (DMSO- $\left.d_{6}, 200 \mathrm{MHz}\right): \delta=4.24-4.34\left(\mathrm{~m} 2 \mathrm{H}, \mathrm{OCH}_{2}\right.$ ), 5.06-5.16 (m, 1H, OH), $6.97\left(\mathrm{~d}, J_{3}=7.0 \mathrm{~Hz}, 1 \mathrm{H}\right), 7.09(\mathrm{t}$, $J_{3}=7.6 \mathrm{~Hz}, 1 \mathrm{H}, \mathrm{H} 5$ '), 7.20-7.39 (m, 7H), 7.39-7.57 (m, $4 \mathrm{H}), 10.24(\mathrm{~s}, 1 \mathrm{H}, \mathrm{NH}) \mathrm{ppm} ;{ }^{13} \mathrm{C}$ NMR (DMSO- $d_{6}$, $50 \mathrm{MHz}): \delta=62.8\left(\mathrm{t}, \mathrm{CH}_{2}\right), 118.4(\mathrm{~d}, \mathrm{C} 2 *), 118.9(\mathrm{~d}$, C6'*), 121.4 (d, C4'*), 121.8 (d, C2'" \& C6'”), 123.9 (d, C2" \& C6"), 125.7 (d, C4'”), 128.0 (d, C5'), 129.5 (d, C3 "”* \& C5'”*), 129.6 (d, C3"* \& C5"*), 129.9 (s, C4”), 138.0 (s, C1'), 143.0 (s, C3'), 150.7 (s, C1”), 151.8 (s, C1'”), 165.9 (s, C2) ppm, C4 \& C6 not visible.

\section{[3-[[4-(3-Chlorophenoxy)-6-phenoxy-1,3,5-triazin-2-yl]ami-}

no]phenyl]methanol $\quad\left(9 \mathrm{~d}, \quad \mathrm{C}_{22} \mathrm{H}_{17} \mathrm{CIN}_{4} \mathrm{O}_{3}\right)$ Prepared according to general procedure $\mathrm{E}$ using $34 \mathrm{mg}$ methyl aminobenzoate $5 d$ ( $0.075 \mathrm{mmol}, 1.00$ equiv.), $0.45 \mathrm{~cm}^{3}$ DIBAL-H (0.385 mmol, 5.10 equiv.); $70{ }^{\circ} \mathrm{C}$ for $1.75 \mathrm{~h}$. After work-up as described in general procedure $\mathrm{E}$, the compound was purified by column chromatography (MPLC, $8.5 \mathrm{~g}$ silica, $8 \mathrm{~cm}^{3} \mathrm{~min}^{-1}$ flow rate, LP/EtOAc 1:1) and dried in vacuo. Yield: $25 \mathrm{mg}(78 \%)$; appearance: colorless oil; $R_{f}=0.49$ (hexane/EtOAc, 1:1); ${ }^{1} \mathrm{H}$ NMR (DMSO- $\left.d_{6}, 200 \mathrm{MHz}\right): \delta=4.30\left(\mathrm{~d}, J_{3}=4.6 \mathrm{~Hz}, 2 \mathrm{H}\right.$, $\left.\mathrm{OCH}_{2}\right), \quad 5.11\left(\mathrm{t}, \quad J_{3}=5.4 \mathrm{~Hz}, \quad 1 \mathrm{H}, \quad \mathrm{OH}\right), 6.97 \quad(\mathrm{~d}$, $\left.J_{3}=7.5 \mathrm{~Hz}, 1 \mathrm{H}\right), 7.10\left(\mathrm{t}, J_{3}=7.7 \mathrm{~Hz}, 1 \mathrm{H}, \mathrm{H} 5\right.$ '), $7.20-7.41$ $(\mathrm{m}, 7 \mathrm{H}), 7.41-7.55(\mathrm{~m}, 4 \mathrm{H}), 10.27(\mathrm{~s}, 1 \mathrm{H}, \mathrm{NH}) \mathrm{ppm} ;{ }^{13} \mathrm{C}$ NMR (DMSO- $\left.d_{6}, 50 \mathrm{MHz}\right): \delta=62.7\left(\mathrm{t}, \mathrm{CH}_{2}\right), 118.4(\mathrm{~d}$, C2'*), 118.9 (d, C6*), 120.8 (d, C6"), 121.4 (d, C4**), 121.7 (d, C2'” \& C6"”), 122.3 (d, C2"), 125.6 (d, C4'”*), 125.8 (d, C4"*), 128.0 (d, C5'), 129.5 (d, C3'” \& C5'”), 130.9 (d, C5”), 133.3 (s, C3"), 138.0 (s, C1'), 142.9 (s, C3'), 151.8 (s, C1'”), 152.5 (s, C1"), 165.9 (s, C2) ppm, C4 \& C6 not visible.

[3-[[4-(2-Chlorophenoxy)-6-phenoxy-1,3,5-triazin-2-yl]amino]phenyl]methanol $\left(9 \mathrm{e}, \mathrm{C}_{22} \mathrm{H}_{17} \mathrm{ClN}_{4} \mathrm{O}_{3}\right)$ Prepared according to general procedure $\mathrm{E}$ using $34 \mathrm{mg}$ methyl aminobenzoate 5e $(0.075 \mathrm{mmol}, 1.00$ equiv. $), 0.35 \mathrm{~cm}^{3}$ DIBAL-H (0.30 mmol, 4.00 equiv.); $-70{ }^{\circ} \mathrm{C}$ for $2 \mathrm{~h}$; then more DIBAL-H $\left(0.10 \mathrm{~cm}^{3}, 0.085 \mathrm{mmol}, 1.15\right.$ equiv. $)$; $70{ }^{\circ} \mathrm{C}$ for $1.75 \mathrm{~h}$. After work-up as described in general procedure $\mathrm{E}$, the compound was purified by column chromatography (MPLC, $8.5 \mathrm{~g}$ silica, $15 \mathrm{~cm}^{3} \mathrm{~min}^{-1}$ flow rate, LP/EtOAc 3: 5) and dried in vacuo. Yield: $32 \mathrm{mg}$ (quant.); 
appearance: colorless solid; m.p.: $52-54{ }^{\circ} \mathrm{C} ; R_{f}=0.44$ (hexane/EtOAc, 1:1); ${ }^{1} \mathrm{H}$ NMR (DMSO- $d_{6}, 200 \mathrm{MHz}$ ): $\delta=4.27\left(\mathrm{~d}, J_{3}=5.3 \mathrm{~Hz}, 2 \mathrm{H}, \mathrm{OCH}_{2}\right), 5.09\left(\mathrm{t}, J_{3}=5.6 \mathrm{~Hz}\right.$, $1 \mathrm{H}, \mathrm{OH}), 6.92-7.12(\mathrm{~m}, 2 \mathrm{H}), 7.17-7.53(\mathrm{~m}, 10 \mathrm{H}), 7.63(\mathrm{~d}$, $\left.J_{3}=7.4 \mathrm{~Hz}, 1 \mathrm{H}\right), 10.31(\mathrm{~s}, 1 \mathrm{H}, \mathrm{NH}) \mathrm{ppm} ;{ }^{13} \mathrm{C} \mathrm{NMR}$ $\left(\mathrm{DMSO}-d_{6}, 50 \mathrm{MHz}\right): \delta=62.8\left(\mathrm{t}, \mathrm{CH}_{2}\right), 118.3\left(\mathrm{~d}, \mathrm{C} 2{ }^{*}\right)$, $118.8\left(\mathrm{~d}, \mathrm{C} 6{ }^{*}\right), 121.4\left(\mathrm{~d}, \mathrm{C} 4{ }^{*}\right), 121.8$ (d, C2'" \& C6"'), 124.1 (d, C6"), 125.7 (d, C4"”), 125.9 (s, C2"), 127.4 (d, C4"), 127.9 (d, C5'), 128.5 (d, C5"), 129.5 (d, C3'” \& C5'”), 130.2 (d, C3”), 137.9 (s, C1'), 142.9 (s, C3'), 147.8 (s, C1"), 151.7 (s, C1'”), 165.8 (s, C2) ppm, C4 \& C6 not visible.

\section{[3-[[4-[4-(1,1-Dimethylethyl)phenoxy]-6-phenoxy-1,3,5-tri-} azin-2-yl]amino]phenyl]methanol (9f, $\mathrm{C}_{26} \mathrm{H}_{26} \mathrm{~N}_{4} \mathrm{O}_{3}$ ) Prepared according to general procedure $\mathrm{E}$ using $35 \mathrm{mg}$ methyl aminobenzoate $\mathbf{5 f}(0.075 \mathrm{mmol}, 1.00$ equiv.), $0.45 \mathrm{~cm}^{3}$ DIBAL-H (0.385 mmol, 5.10 equiv.); $-70{ }^{\circ} \mathrm{C}$ for $1.5 \mathrm{~h}$. After work-up as described in general procedure $\mathrm{E}$, the compound was dried in vacuo and did not require further purification. Yield: $33 \mathrm{mg}$ (quant.); appearance: colorless solid; m.p.: $47-50{ }^{\circ} \mathrm{C} ; R_{f}=0.54$ (hexane/EtOAc, $1: 1) ;{ }^{1} \mathrm{H}$ NMR (DMSO- $d_{6}, 200 \mathrm{MHz}$ ): $\delta=1.31$ (s, 9H, $\left.\left(\mathrm{CH}_{3}\right)_{3}\right), 4.28\left(\mathrm{~d}, J_{3}=4.5 \mathrm{~Hz}, 2 \mathrm{H}, \mathrm{OCH}_{2}\right), 5.11(\mathrm{bs}, 1 \mathrm{H}$, $\mathrm{OH}), 6.95\left(\mathrm{~d}, J_{3}=7.5 \mathrm{~Hz}, 1 \mathrm{H}\right), 7.06\left(\mathrm{t}, J_{3}=7.7 \mathrm{~Hz}, 1 \mathrm{H}\right.$, $\mathrm{H}^{\prime}$ ) , 7.12-7.21 (m, 2H), 7.22-7.34 (m, 4H), 7.35-7.52 (m, $5 \mathrm{H}), 10.20(\mathrm{~s}, 1 \mathrm{H}, \mathrm{NH}) \mathrm{ppm} ;{ }^{13} \mathrm{C} \mathrm{NMR}\left(\mathrm{DMSO}-d_{6}\right.$, $50 \mathrm{MHz}): \delta=31.2\left(\mathrm{q}, \mathrm{C}\left(\mathrm{CH}_{3}\right)_{3}\right), 34.2\left(\mathrm{~s}, \mathrm{C}\left(\mathrm{CH}_{3}\right)_{3}\right), 62.8$ (t, $\left.\mathrm{CH}_{2}\right), 118.3\left(\mathrm{~d}, \mathrm{C} 2{ }^{\prime} *\right), 118.8\left(\mathrm{~d}, \mathrm{C} 6{ }^{*}\right), 121.1$ (d, C2"* \& C6"*), $121.3\left(\mathrm{~d}, \mathrm{C} 4{ }^{*}\right), 121.8$ (d, C2'” \& C6" ") 125.6 (d, C4" "), 126.2 (d, C3" \& C5"), 127.9 (d, C5'), 129.5 (d, C3"” \& C5"”), 138.1 (s, C1'), 142.9 (s, C3'), 147.9 (s, C4"*), 149.5 (s, C1"*), 151.8 (s, C1 ${ }^{\prime \prime}$ ), 165.9 (s, C2) ppm, C4 \& C6 not visible.

\section{[4-[[4-(4-Methoxyphenoxy)-6-phenoxy-1,3,5-triazin-2-yl]a-} mino]phenyl]methanol $\quad\left(9 \mathrm{~g}, \quad \mathrm{C}_{23} \mathrm{H}_{20} \mathrm{~N}_{4} \mathrm{O}_{4}\right)$ Prepared according to general procedure $\mathrm{E}$ using $33 \mathrm{mg}$ methyl aminobenzoate $5 \mathrm{~g}$ (0.075 mmol, 1.00 equiv.), $0.45 \mathrm{~cm}^{3}$ DIBAL-H (0.385 mmol, 5.10 equiv.); $-70{ }^{\circ} \mathrm{C}$ for $1.75 \mathrm{~h}$. After work-up as described in general procedure $\mathrm{E}$, the compound was dried in vacuo and did not require further purification. Yield: $31 \mathrm{mg}$ (quant.); appearance: colorless oil; $R_{f}=0.32$ (hexane/EtOAc, $1: 1$ ); ${ }^{1} \mathrm{H}$ NMR (DMSO- $d_{6}$, $200 \mathrm{MHz}): \delta=3.78\left(\mathrm{~s}, 3 \mathrm{H}, \mathrm{OCH}_{3}\right), 4.28\left(\mathrm{~d}, J_{3}=5.7 \mathrm{~Hz}\right.$, $\left.2 \mathrm{H}, \mathrm{CH}_{2}\right), 5.10\left(\mathrm{t}, J_{3}=5.7 \mathrm{~Hz}, 1 \mathrm{H}, \mathrm{OH}\right), 6.91-7.52(\mathrm{~m}$, $13 \mathrm{H}), 10.18(\mathrm{~s}, 1 \mathrm{H}, \mathrm{NH}) \mathrm{ppm} ;{ }^{13} \mathrm{C}$ NMR (DMSO- $d_{6}$, $50 \mathrm{MHz}): \delta=55.4\left(\mathrm{q}, \mathrm{OCH}_{3}\right), 62.8\left(\mathrm{t}, \mathrm{CH}_{2}\right), 114.4$ (d, C3" \& C5"), 118.3 (d, C2'*), 118.8 (d, C6'*), 121.2 (d, C4'*), 121.8 (d, C2" " \& C6" "), 122.6 (d, C2" \& C6"), 125.6 (d, C4"”), 127.9 (d, C5'), 129.5 (d, C3"” \& C5"”), 138.1 (s, C1'), 142.9 (s, C3'), 145.2 (s, C1'), 151.8 (s, C1'”), 156.8 (s, C4”), 165.9 (s, C2) ppm, C4 \& C6 not visible.
Acknowledgements Open access funding provided by TU Wien (TUW). Funding from the Austria Wirtschaftsservice (Uni Invent Prize: Cardiogenic Drug Candidate) is gratefully acknowledged.

Open Access This article is distributed under the terms of the Creative Commons Attribution 4.0 International License (http://creative commons.org/licenses/by/4.0/), which permits unrestricted use, distribution, and reproduction in any medium, provided you give appropriate credit to the original author(s) and the source, provide a link to the Creative Commons license, and indicate if changes were made.

\section{References}

1. Wobus AM, Boheler KR (2005) Physiol Rev 85:635

2. Plowright AT, Engkvist O, Gill A, Knerr L, Wang Q-D (2014) Angew Chem Int Ed 53:4056

3. Pandian GN, Sato S, Anandhakumar C, Taniguchi J, Takashima K, Syed J, Han L, Saha A, Bando T, Nagase H, Sugiyama H (2014) ACS Chem Biol 9:2729

4. Davies SG, Kennewell PD, Russell AJ, Seden PT, Westwood R, Wynne GM (2015) J Med Chem 58:2863

5. Sahara M, Santoro F, Chien KR (2015) EMBO J 34:710

6. World Health Organization (2008) 2008-2013 Action plan for the global strategy for the prevention and control of noncommunicable diseases. WHO, Geneva

7. Lloyd-Jones D, Adams RJ, Brown TM, Carnethon M, Dai S, De Simone G, Ferguson TB, Ford E, Furie K, Gillespie C, Go A, Greenlund K, Haase N, Hailpern S, Ho PM, Howard V, Kissela B, Kittner S, Lackland D, Lisabeth L, Marelli A, McDermott MM, Meigs J, Mozaffarian D, Mussolino M, Nichol G, Roger VL, Rosamond W, Sacco R, Sorlie P, Stafford R, Thom T, Wasserthiel-Smoller S, Wong ND, Wylie-Rosett J (2010) Circulation 121:e46

8. Boheler KR, Czyz J, Tweedie D, Yang H-T, Anisimov SV, Wobus AM (2002) Circ Res 91:189

9. Murry CE, Field LJ, Menasche P (2005) Circulation 112:3174

10. Forrester JS, White AJ, Matsushita S, Chakravarty T, Makkar RR (2009) JACC Cardiovasc Interv 2:1

11. Ausoni S, Sartore S (2009) J Cell Biol 184:357

12. Cyranoski D (2007) Nature 450:462

13. Cyranoski D (2008) Nature 452:406

14. Yamanaka S (2009) Cell 137:13

15. Wu X, Ding S, Ding Q, Gray NS, Schultz PG (2004) J Am Chem Soc $126: 1590$

16. Boer PH (1993) Exp Cell Res 207:421

17. Ruskoaho H (2003) Endocr Rev 24:341

18. Koley M, König X, Hilber K, Schnürch M, Stanetty P, Mihovilovic MD (2011) Arkivoc 2011:45

19. Koley M, Mike AK, Heher P, Koenig X, Schön M, Schnürch M, Hilber K, Weitzer G, Mihovilovic MD (2013) Med Chem Comm 4:1189

20. Mike AK, Koenig X, Koley M, Heher P, Wahl G, Rubi L, Schnürch M, Mihovilovic MD, Weitzer G, Hilber K (2014) Cell Physiol Biochem 33:205

21. Gardner DG, Chen S (1999) Life Sci 65:1607

22. Pakala R, Davies PJA, Chandraratna R, Benedict CR (1995) J Am Coll Cardiol 25:83A

23. Hayashi A, Suzuki T, Tajima S (1995) J Biochem 117:132

24. Miano JM, Topouzis S, Majesky MW, Olson EN (1996) Circulation 93:1886

25. Peclo MM, Printseva OY (1987) Experientia 43:196 
26. Wobus AM, Kaomei G, Shan J, Wellner M-C, Rohwedel J, Ji G, Fleischmann B, Katus HA, Hescheler J, Wolfgang-Michael F (1997) J Mol Cell Cardiol 29:1525

27. Bempong DK, Honigberg IL, Meltzer NM (1995) J Pharmaceut Biomed 13:285

28. Kojima R, Fujimori T, Kiyota N, Toriya Y, Fukuda T, Ohashi T, Sato T, Yoshizawa Y, Takeyama K-i, Mano H, Masushige S, Kato S (1994) J Biol Chem 269:32700

29. Theodosiou M, Laudet V, Schubert M (2010) Cell Mol Life Sci 67:1563

30. Duester G (2008) Cell 134:921

31. Sachidanandan C, Yeh Jing-Ruey J, Peterson QP, Peterson RT (2008) PLoS ONE 3:e1947

32. Eicher T, Hauptmann S (2003) The chemistry of heterocycles. Wiley, Weinheim, p 446

33. Goetz RJ, Robertazzi A, Mutikainen I, Turpeinen U, Gamez P, Reedijk J (2008) J Chem Commun 2008:3384

34. Sun L, Guo Y, Peng G, Li C (2008) Synthesis 2008:3487

35. Sadek H, Hannack B, Choe E, Wang J, Latif S, Garry MG, Garry DJ, Longgood J, Frantz DE, Olson EN, Hsieh J, Schneider JW (2008) Proc Natl Acad Sci USA 105:6063

36. Beech WF (1967) J Chem Soc C 1967:466

37. Allington RD, Attwood D, Hamerton I, Hay JN, Howlin B (2002) J Polym 44:793

38. Theodorou V, Skobridis K, Tzakos AG, Ragoussis V (2007) Tetrahedron Lett 48:8230

39. Kocienski PJ (1994) Protecting groups, vol 1. Thieme, Stuttgart

40. Wender PA, Schaus JM, White AW (1980) J Am Chem Soc 102:6157

41. Dippy JFJ, Hughes SRC, Rozanski A (1959) J Chem Soc 1959:2492
42. Chandrasekaran S, Kluge AF, Edwards JA (1977) J Org Chem 42:3972

43. Braude EA, Nachod FC (1955) Determination of organic structures by physical methods. Academic, New York

44. Wuts PGM (2014) Greene's protective groups in organic synthesis, 5th edn. Wiley, Hoboken

45. Yang C, Pittman CU Jr (1998) Synth Commun 28:2027

46. Feng J-C, Liu B, Dai L, Yang X-L, Tu S-J (2001) Synth Commun $31: 1875$

47. Brown HC, Narasimhan S, Choi YM (1982) J Org Chem 47:4702

48. Mihovilovic MD, Schnürch M, Hilber K, Koenig X, Linder T, Mike A (2013) Triazine derivatives as differentiation accelerators. PCT Int. Appl. WO 2013040622 A1, Mar 28, 2013; Chem Abstr 158:465140

49. Mihovilovic MD, Schnürch M, Hilber K, Koenig X, Linder T, Mike A (2012) Triazine derivatives as differentiation accelerators. Austrian Pat. Appl. [Post-Grant] AT 511441 A4, Dec 15, 2012; Chem Abstr 161:269346

50. Hoye TR, Aspaas AW, Eklov BM, Ryba TD (2005) Org Lett 7:2205

51. Gottlieb HE, Kotlyar V, Nudelman A (1997) J Org Chem 62:7512

52. Bruckner H, Wachsmann M (2003) J Chromatogr A 998:73

53. Chaudhari JA, Patel RP, Hathi MV (2007) E-J Chem 4:385

54. von Angerer S (2004) In: Weinreb SM (ed) Product subclass 3: 1,3,5-triazines and phosphorus analogues. Science of synthesis, vol 17. Georg Thieme Verlag KG, Stuttgart, p 449

55. Spray DC, Campos de Carvalho AC, Mendez-Otero R (2010) Stem Cells Dev 19:403 